\title{
The neural basis of drug craving: an incentive-sensitization theory of addiction
}

\author{
Terry E. Robinson and Kent C. Berridge \\ Department of Psychology and Neuroscience Program, The University of Michigan, Neuroscience Laboratory Building, Ann Arbor, \\ MI 48104-1687 (USA)
}

(Accepted 20 April 1993)

Key words: Drug addiction; Brain; Dopamine; Incentive motivation; Sensitization; Neuroadaptation; Nucleus accumbens; Striatum

This paper presents a biopsychological theory of drug addiction, the 'Incentive-Sensitization Theory'. The theory addresses three fundamental questions. The first is: why do addicts crave drugs? That is, what is the psychological and neurobiological basis of drug eraving? The second is: why does drug craving persist even after long periods of abstinence? The third is whether 'wanting' drugs (drug craving) is attributable to 'liking' drugs (to the subjective pleasurable effects of drugs)? The theory posits the following. (1) Addictive drugs share the ability to enhance mesotelencephalic dopamine neurotransmission. (2) One psychological function of this neural system is to attribute 'incentive salience' to the perception and mental representation of events associated with activation of the system. Incentive salience is a psychological process that transforms the perception of stimuli, imbuing them with salience, making them attractive, 'wanted', incentive stimuli. (3) In some individuals the repeated use of addictive drugs produces incremental neuroadaptations in this neural system, rendering it increasingly and perhaps permanently, hypersensitive ('sensitized') to drugs and drug-associated stimuli. The sensitization of dopamine systems is gated by associative learning, which causes excessive incentive salience to be attributed to the act of drug taking and to stimuli associated with drug taking. It is specifically the sensitization of incentive salience, therefore, that transforms ordinary 'wanting' into excessive drug craving. (4) It is further proposed that sensitization of the neural systems responsible for incentive salience (for 'wanting') can occur independently of changes in neural systems that mediate the subjective pleasurable effects of drugs (drug 'liking') and of neural systems that mediate withdrawal. Thus, sensitization of incentive salience can produce addictive behavior (compulsive drug seeking and drug taking) even if the expectation of drug pleasure or the aversive properties of withdrawal are diminished and even in the face of strong disincentives, including the loss of reputation, job, home and family. We review evidence for this view of addiction and discuss its implications for understanding the psychology and neurobiology of addiction.

\section{CONTENTS}

Summary

1. Introduction

2. The Incentive-Sensitization Theory of Addiction: an overview

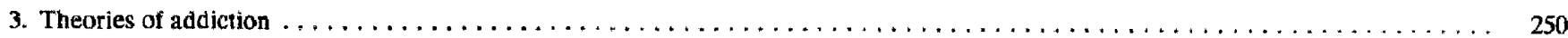

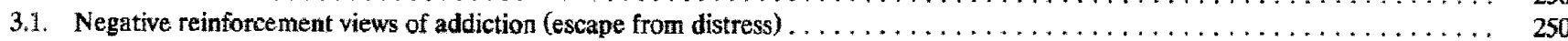

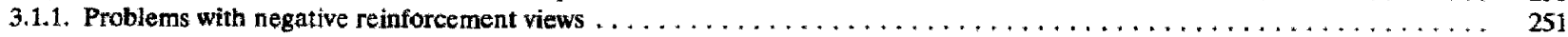

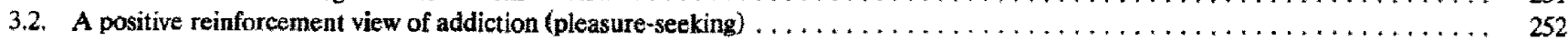

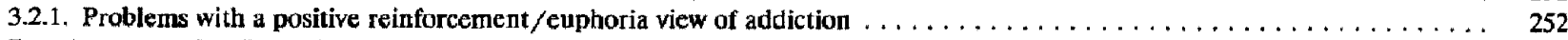

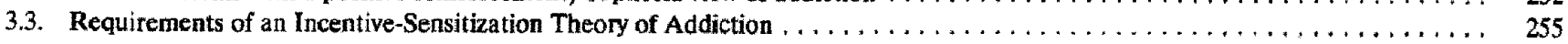

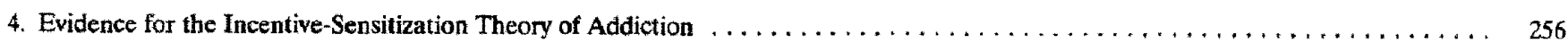

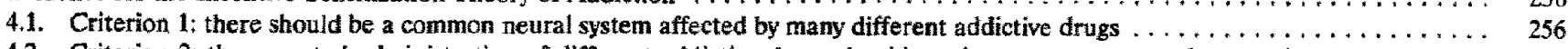

4.2. Criterion 2 : the repeated administration of different addietive drugs should render a common neural system hypersemsitive in a

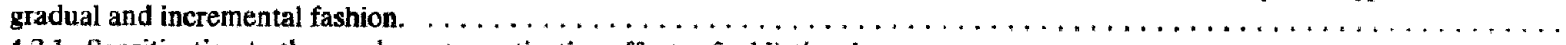

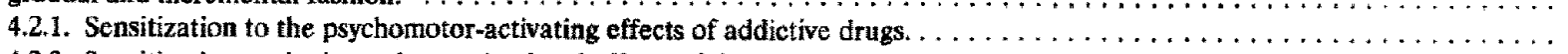

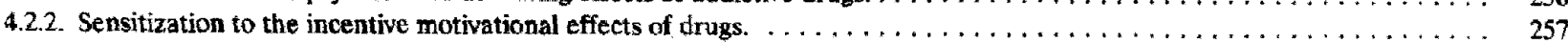

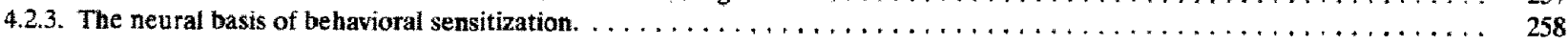


4.3. Criterion 3: sensitization-related neuroadaptations should be very long-lasting. . . . . . . . . . . . . . . . .

4.4. Criterion 4: the expression of sensitization-related neuroadaptations should be amenable to conditioned stimulus control. . . . . 259

4.5. Criterion 5 : the role of mesotelencephalic dopamine systems in incentive motivation. . . . . . . . . . . . . . 60

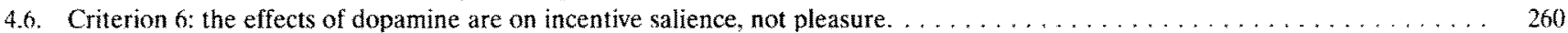

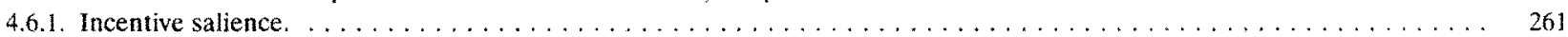

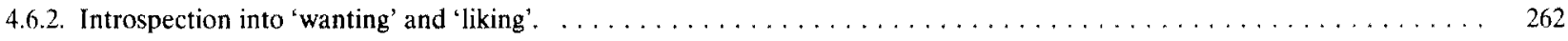

4.63. Evidence that mesotelencephalic dopamine mediates incentive salience, not pleasure. . . . . . . . . . . . . . . 262

5. Elaboration of the Incentive-Sensitization Theory and its implications. . . . . . . . . . . . . . . . . . . . . . . 265

5.1. The independence of drug craving from drug pleasure and withdrawal. $\ldots \ldots \ldots \ldots$

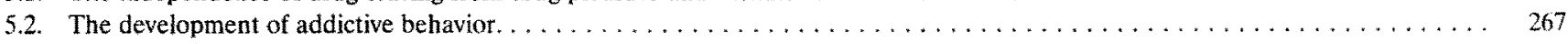

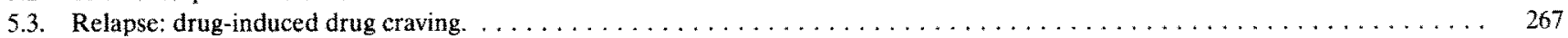

5.4. Relapse: interactions between different drugs and the effects of drug-related stimuli. . . . . . . . . . . . . . . . 268

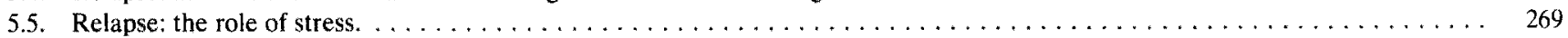

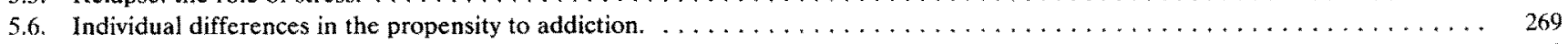

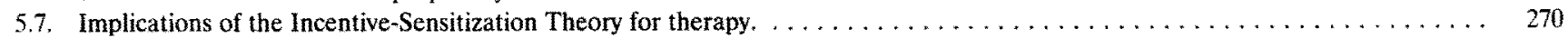

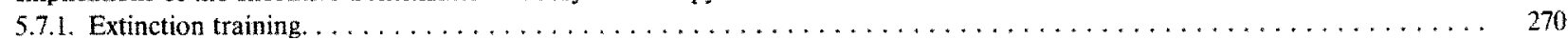

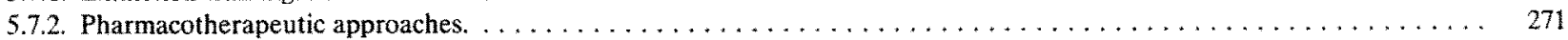

5.8. Relationship between incentive sensitization and other views of addiction. . . . . . . . . . . . . . . . .

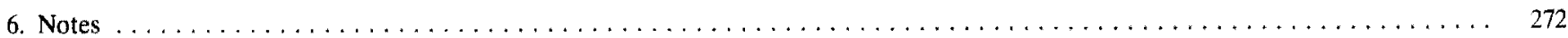

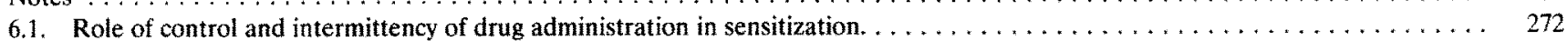

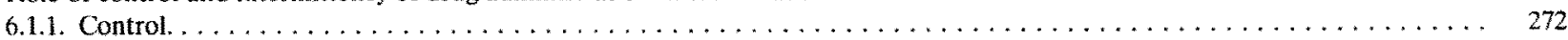

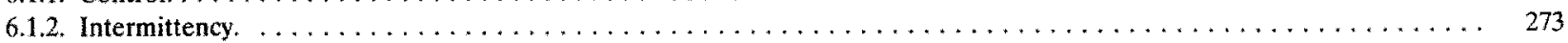

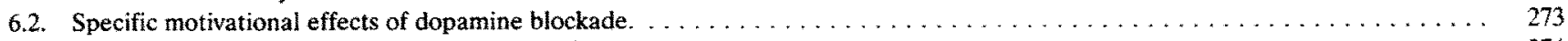

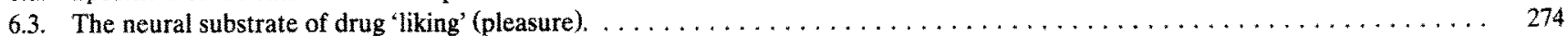

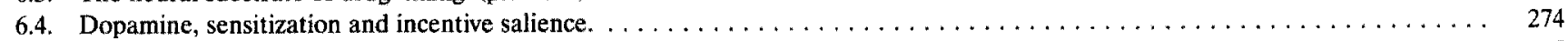

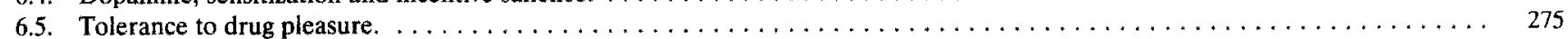

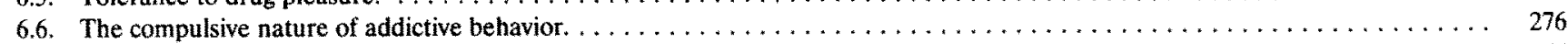

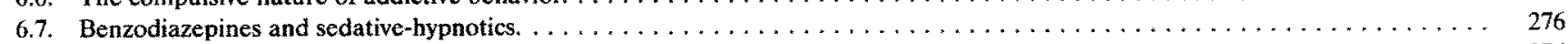

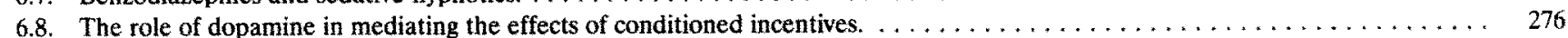

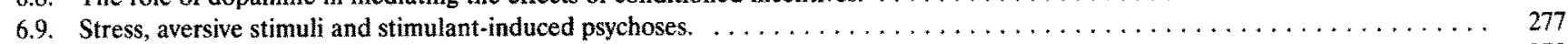

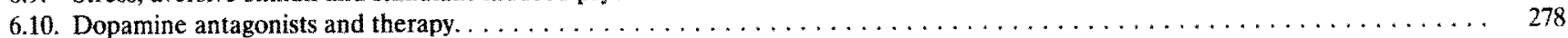

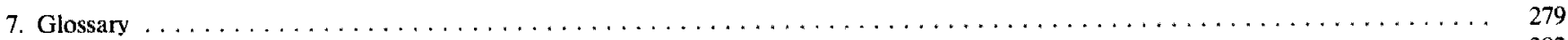

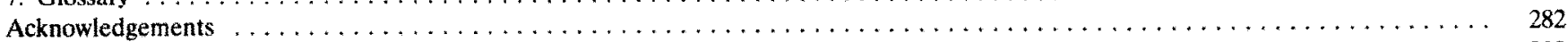

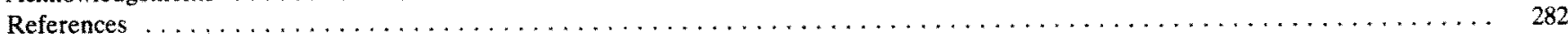

A firm conviction of the material reality of Hell never prevented medieval Christians from doing what their ambition, lust or covetousness suggesied. Lung cancer, traffic accidents and the millions of miserable and misery-creating alcoholics are facts even more certain than was, in Dante's day, the fact of the Inferno. But all such facts are remote and unsubstantial when compared with the near, felt fact of a craving, here and now, for release or sedation, for a drink or a smoke.

(Aldous Huxley, The Doors of Perception, 1951)

\section{INTRODUCTION}

There are three major features of addictive behavior that need to be explained by any adequate theory of drug addiction*. The first is drug cravingt, by which we simply mean intensely 'wanting' $\dagger$ drugs ${ }^{184}$. Although drug addiction $\dagger$ is defined as a pattern of 'compulsive drug-taking behavior', drug taking does not in itself constitute addictive behavior. Only when the repeated self-administration of drugs leads to a pattern of compulsive drug-seeking and drug-taking behavior, which occurs at the expense of most other activities, is a person said to be addicted ${ }^{79,148}$. To understand addiction, therefore, we need to under- stand the process by which drug-taking behavior evolves into compulsive drug-taking behavior. Presumably this transformation in behavior occurs because addicts develop an obsessive craving for drugs, a craving that is so irresistible that it almost inevitably leads to drug seeking and drug taking. It is difficult, of course, to provide an adequate definition of subjective terms, such as 'wanting' and craving ${ }^{184,363}$, but clinical experience suggests that drug craving is fundamental to addiction; it cannot be ignored. Any satisfactory account of addiction must explain: why do addicts want or crave drugs so much?

Drug addiction is also "a chronic relapsing disorder" (ref. 148, p. 522). The second major feature of addic-

\footnotetext{
* Many of the terms in this article are used in different ways by different authors and they are not always clearly defined. To avoid ambiguity we have provided a glossary with definitions of many of the most problematic terms. Thus, a reference to the glossary, which will be indicated by the symbol, $\uparrow$, refers the reader to the definition of a term. The reader may not always agree with a given definition, but we hope at least this makes it clear what we mean.
} 
tion that must be explained, therefore, is: why drug craving often persists or can be reinstated, long after the discontinuation of drug use. An understanding of the propensity to relapse will be critical not only for understanding the process of addiction, but in developing effective therapies.

A third feature of drug addiction that requires explanation is that, as drugs come to be 'wanted' moreand-more, they often come to be 'liked' less-and-less. That is, as craving for drugs increases the pleasure derived from drugs often decreases. Why is this? What is the relationship between 'wanting' drugs and 'liking' drugs and does this relationship change during addiction?

The purpose of this article is to present a biopsychological theory of addiction, an Incentive-Sensitization Theory, that addresses these issues *. The paper is organized into four parts. In Part I the theory is summarized to give a brief overview of its major features. In Part II the theory is put into a broader context by critically discussing other theories of addiction, specifically negative reinforcement (e.g., withdrawal avoidance) and positive reinforcement (e.g., pleasure-seeking) theories. In doing so it is argued that theories based on the concepts of negative or positive reinforcement $\dagger$ do not adequately explain the key features of addiction discussed above. In Part III research findings that support the concept of an IncentiveSensitization Theory are reviewed. Finally, in Part IV the theory is elaborated and its implications discussed in greater detail.

\section{THE INCENTIVE-SENSITIZATION THEORY OF ADDICTION: AN OVERVIEW}

The Incentive-Sensitization Theory of Addiction posits that addictive behavior is due largely to progressive and persistent neuroadaptations caused by repeated drug use. It is, if you will, a 'neuroadaptationist model'. It is proposed that these drug-induced changes in the nervous system are manifest both neurochemically and behaviorally by the phenomenon of 'sensitization', which refers to a progressive increase in a drug effect with repeated treatment ${ }^{272,291}$. These sensitization-related neuroadaptations have not been considered in previous theories of addiction. In fact, until recently, the phenomenon of sensitization usually was not mentioned in books and articles on addiction and if sensitization was mentioned, it was referred to only in passing, as part of a more extensive discussion of tolerance. Nevertheless, it is proposed here that the defining characteristics of addiction (craving and relapse) are due directly to drug-induced changes in those functions normally subserved by a neural system that undergoes sensitization-related neuroadaptations.

The neural system that is rendered hypersensitive ('sensitized') to activating stimuli is hypothesized to mediate a specific psychological function involved in the process of incentive motivation: namely the attribution of incentive saliencet to the perception and mental representation of stimuli and actions. This makes stimuli and their representations highly salient, attractive and 'wanted'. It is the activation of this neural system that results in the experience of 'wanting', and transforms ordinary stimuli into incentive stimuli.

Sensitization of this neural system by drugs results in a pathological enhancement in the incentive salience that the nervous system attributes to the act of drug taking. The co-activation of associative learning directs the focus of this neurobehavioral system to specific targets that are associated with drugs and leads to an increasing pathological focus of incentive salience on drug-related stimuli. Thus, with repeated drug use the act of drug taking and drug-associated stimuli, gradually become more and more attractive. Drug-associated stimuli become more and more able to control behavior, because the neural system that mediates 'wanting' becomes progressively sensitized. 'Wanting' evolves into obsessive craving and this is manifest behaviorally as compulsive drug seeking and drug taking. Therefore, by this view, drug craving and addictive behavior are due specifically to sensitization of incentive salience.

But 'wanting' is not 'liking'. The neural system responsible for 'wanting' incentives is proposed to be separable from those responsible for 'liking' incentives (i.e., for mediating pleasure) and repeated drug use only sensitizes the neural system responsible for 'wanting'. Because of this, addictive behavior is fundamentally a problem of sensitization-induced excessive 'wanting' alone. This is in contrast to 'pleasure-seeking' theories of addiction, which explicitly assume that the incentive motivational properties of drugs are due directly to their subjective pleasurable effects; i.e., their ability to produce positive affective states. In colloquial language, it is usually assumed that addicts 'want' drugs because they 'like' drugs and the more they 'like'

\footnotetext{
* This paper is not a comprehensive review of the primary research literature on addiction and addictive drugs. We cite review articles to support specific points in many instances. Readers should consult these review articles for more extensive lists of citations to the primary literature.
} 
them the more they should 'want' them. In this traditional view 'wanting' and 'liking' drugs are necessarily connected. The Incentive-Sensitization Theory is unique, however, because we propose the progressive increase in drug 'wanting' that characterizes addiction is not accompanied by an increase in the pleasure derived from drugs. Repeated drug use does not sensitize neural systems responsible for the subjective pleasurable $\dagger$ effects of drugs, only those responsible for incentive salience - transforming 'wanting' into craving.

In addition, the neuroadaptations underlying behavioral sensitization are long-lasting and in some cases they may be permanent. It is hypothesized that it is the persistence of sensitization-related neuroadaptations that renders addicts hypersensitive to drugs and to drug-related stimuli, even after years of abstinence. It is the permanence of sensitization that is thought to render drug-related stimuli so effective in precipitating relapse, even in detoxified, 'recovered' addicts.

Finally, it is hypothesized that the neural substrate for incentive-sensitization (that is the neural system(s) that normally attributes salience to incentive stimuli and becomes sensitized by addictive drugs) is the mesotelencephalic dopamine system. Sensitization results in an increase in the responsiveness of the dopamine system to activating stimuli, such that activating stimuli produce a greater increase in dopamine neurotransmission in sensitized than in non-sensitized individuals. The relationship between changes in dopamine neurotransmission, the subjective pleasurable effects of drugs and incentive salience, which occurs during addiction according to the IncentiveSensitization Theory, are illustrated schematically in Fig. 1.

The Incentive-Sensitization Theory of Addiction will be discussed in much greater detail later. Before that, however, we need to address the features of addictive behavior that are not adequately explained by other theories of addiction and thus require explanation by a new theory. The most widely accepted theories of addiction presently fall into two classes: negative reinforcement models (e.g., drugs are taken to avoid the symptoms of withdrawal) and positive reinforcement models (i.e., drugs reinforce self-administration behavior by producing pleasure). There have been many papers describing the strengths and short-comings of negative and positive reinforcement views of addiction $^{86,326,363,365}$ and it is not necessary to review this entire literature in great detail here. Instead, negative and positive reinforcement models will be only briefly summarized and their difficulty in explaining several key features of addiction (e.g., craving and relapse) will

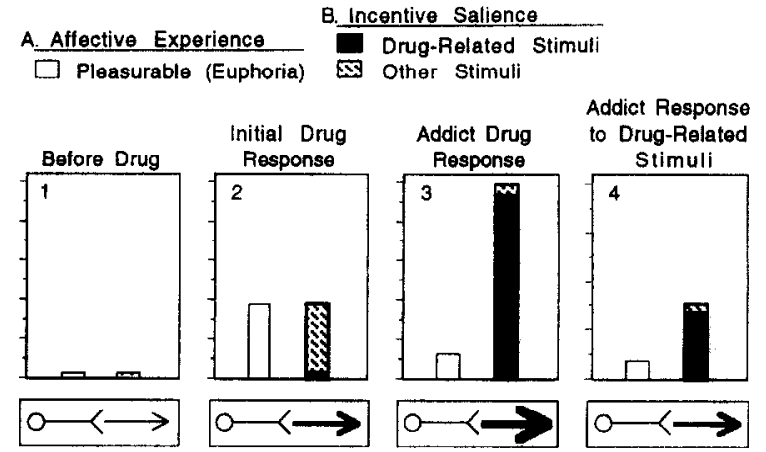

Fig. 1. A schematic illustration of changes, as addiction develops, in (A) the pleasurable affective experience produced by drugs; (B) the incentive salience attributed to drug-associated stimuli and their mental representations and to other stimuli; and (C) the activation of dopamine systems (arrows); as proposed by Incentive-Sensitization. Dopamine systems are hypothesized to provide the neural substrate for incentive salience. Panel 1 depicts the subjective experience and degree of incentive salience in a normal individual (before taking a drug). We assume the person is not depressed and therefore, the normal affective state is fairly neutral, but slightly in the positive direction (as is incentive salience). Panel 2 indicates that the initial drug experience (or first few drug experiences) results in a marked increase in subjective pleasure (the drug is 'liked') and in the attribution of salience to stimuli in the immediate environment. These are not yet 'drug-related' stimuli because they have not yet been linked associatively with the drug experience. (Note that the initial drug experience could also have aversive components, but these are not depicted here.) The initial drug experience is accompanied by an increase in dopamine neurotransmission, as indicated by an increase in the size of the arrow in the lower panel indicating 'dopamine activity'. Panel 3 shows that after many drug experiences and the development of addictive behavior, the response to the same dose of the drug shown in Panel 2 is changed. In the addict the subjective pleasurable effects of the drug are decreased due to tolerance (alternatively, they could be unchanged, as discussed in Fig. 2 and Note 5 in $\mathrm{Ch}$. 6). But the incentive salience attributed to drug taking is markedly enhanced due to sensitization of the neural system responsible for the attribution of incentive salience. Due to associative conditioning there is a focus of incentive salience on what are now drug-associated stimuli. It is further hypothesized that this sensitization of incentive salience is due to sensitization of dopamine neurotransmission (large arrow in lower panel; but also see Note 4 in Ch. 6). Panel 4 shows that in the addict exposure to conditioned incentive stimuli (stimuli that have acquired incentive value through their association with drugs) may produce effects similar to the drug itself, but of lesser magnitude. It is the sensitization of incentive salience depicted in Panels 3 and 4 that is hypothesized to be responsible for excessive drug 'wanting' (craving) in the addict, leading to compulsive drug-seeking and drug-taking behavior.

be emphasized, primarily to provide a comparison with the Incentive-Sensitization Theory. We want to stress, however, that none of these views are mutually exclusive. Pleasure-seeking, escape from distress and incentive-sensitization probably each play some role in drug-taking behavior.

\section{THEORIES OF ADDICTION}

\subsection{Negative reinforcement views of addiction (escape from distress)}

Historically, the aversive consequences of discontinuing drug use (the withdrawal syndrome) have been a 
central focus of research on addiction, in part because many early studies were on opiates, which produce clear tolerance and physical withdrawal symptoms. This research emphasized the action of drugs as negative reinforcers ${ }^{\dagger 64,148,180,227,312,326,337,349,365}$. To paraphrase Wise and Bozarth ${ }^{365}$, negative reinforcers sustain behavior (drug seeking and drug taking in this case) not because of the state they produce, but because of the state they alleviate. According to this negative reinforcement view of addiction drug use is maintained because the aversive symptoms associated with withdrawal are alleviated by the drug. Addictive drugs that do not result in overt physical withdrawal symptoms, such as cocaine and the amphetamines, are thought to act as negative reinforcers by alleviating a 'psychological distress syndrome' produced by the discontinuation of drug use (ref. 105 for example). In addition, previously neutral environmental stimuli associated with withdrawal can themselves come to elicit withdrawallike symptoms, by secondary conditioning ${ }^{305,306,357}$. Thus, drugs may not only alleviate 'primary' withdrawal symptoms, but also the conditioned withdrawal symptoms induced by exposure to drug-related stimuli. A second negative reinforcement view is that drugs are sometimes used to 'self-medicate', relieving preexistent symptoms such as pain, anxiety or depression that occur in life independent of drug use (ref. 170 for example).

The traditional focus on withdrawal and tolerance was driven by the assumption that these processes are critical for the development and maintenance of addictive behavior. It is now clear, however, that the avoidance of withdrawal is not the most important factor in the development or maintenance of addictive behavior, although certainly the avoidance of withdrawal may motivate drug-seeking and drug-taking behavior in some instances. A number of leading figures in drug addiction research have noted, for example, that "physical dependence is neither a necessary nor sufficient condition for addiction" (ref. 365, p. 470), that "for rats and monkeys physical dependence is neither a necessary nor a sufficient condition for opiates to act as reinforcers" (ref. 290, p. 186) and that "physical dependence is currently viewed not so much as a direct cause of drug dependence but as one of several factors that contribute to its development" (ref. 148, p. 527). A number of critiques of negative reinforcement theories of addiction have been published ${ }^{86,149,326,363,365}$ and the major shortcomings of negative reinforcement theories in explaining addiction are briefly summarized below. 3.1.1. Problems with negative reinforcement views - Both people and animals will self-administer opioids in the absence of withdrawal symptoms or physical dependence ${ }^{335,372}$. For example, Ternes et al. ${ }^{335}$ found that in cynomolgus monkeys the opioid, hydromorphone, maintained self-administration at doses that produced neither tolerance nor physical dependence, the latter indicated by the absence of any effect of a naloxone challenge. Similarly, Lamb et al. ${ }^{192}$ recently reported that former heroin addicts, who showed no withdrawal symptoms upon a naloxone challenge, nevertheless worked at high rates (lever pressed) to receive a low-dose injection of morphine.

- Maximal periods of drug self-administration often do not coincide in time with periods of maximal withdrawal distress ${ }^{365}$. This lack of correlation between withdrawal distress and drug-seeking behavior is also evident in comparisons made across drug classes. Jaffe (ref. 149, p. 9) notes that, although the severity of the withdrawal syndrome associated with different drugs varies dramatically, ranging from very subtle physiological signs to life-threatening consequences, "there is little correlation between the visibility or physiological seriousness of withdrawal signs and their motivational force" in maintaining addictive behavior.

- There are many drugs used medically that produce withdrawal syndromes but "are not typically self-administered for non-medical purposes", including "certain tricyclic antidepressants (imipramine, amitriptyline), anticholinergics and $\kappa$-opioid agonists" (ref. 149, p. 9).

- There are numerous reports that the "relief of withdrawal is minimally effective in treating addiction" (ref. 365, p. 470 for references).

- There is a high tendency to relapse even after an extended period of abstinence from drugs, long after overt withdrawal symptoms have subsided. This is usually explained in the context of conditioned withdrawal effects, whereby environmental stimuli associated with withdrawal come to elicit withdrawal-like symptoms ${ }^{306,357}$. There are, however, a number of problems with this explanation. (1) At least a third of opiatc addicts deny that they experience conditioned withdrawal symptoms when they are exposured to drug-related stimuli ${ }^{50}$. (2) Although many opiate addicts experience conditioned withdrawal symptoms very few cite this as the reason for resuming drug use ${ }^{212}$. There is, in fact, a poor correlation between craving and withdrawal signs $^{50}$. Even withdrawal-like physiological symptoms induced by drug-associated cues (e.g., temperature, skin resistance, heart rate) are not highly correlated with reports of subjective state ${ }^{80}$. (3) Stewart et al. (ref. 326, p. 258) have argued that "attempts to demonstrate such conditioned withdrawal symptoms increase the probability of drug taking and relapse in animals have been unsuccessful" (ref. 329 as well). 
- A number of researchers have noted that self-reported craving for some drugs, such as cocaine, is often highest immediately after drug administration, when the drug is producing subjective pleasure (a 'high') and withdrawal symptoms are eliminated or are at their weakest ${ }^{50,80,96,98,150,215}$. If drug craving were due to a desire to relieve withdrawal symptoms (ref. 64 for example), it would be expected that craving would dramatically decrease when the drug alleviates withdrawal and is producing pleasure, not be sustained or even increase.

- Finally, animals will avidly self-administer a variety of drugs directly into brain regions that do not produce withdrawal symptoms ${ }^{366}$. Furthermore, the infusion of drugs into these same brain regions can 'prime' or reinstate responding in animals in which drug responding has been extinguished ${ }^{326}$. These studies have established that the incentive motivational effects of centrally applied drugs can be dissociated from their negative reinforcing or withdrawal-related effects.

For the reasons summarized above it is now generally accepted that the negative reinforcing effects of drugs are not necessary for the development and maintenance of addictive behavior. Escape from distress cannot explain the defining characteristics of addiction, craving and relapse. We do not wish to imply, however, that withdrawal plays no role at all in the maintenance of addictive behavior. The alleviation of withdrawal distress may indeed sometimes motivate drug-seeking and drug-taking behavior. In addition, the positive reinforcing effects of drugs may be enhanced when drugs are given during periods of withdrawal distress ${ }^{86,290}$. However, relief from withdrawal symptoms cannot be the sole cause or even the primary cause of drug craving and compulsive drug-taking behavior.

\subsection{A positive reinforcement view of addiction (pleasure-seeking)}

In part because of the shortcomings of negative reinforcement theories of addiction more recent formulations have focussed on the role of drugs as positive reinforcers $\dagger^{148,326,349,363,365}$. Most drugs that are self-administered by people also act as positive reinforcers for animals. Thus, a positive reinforcement view of addiction posits that drug self-administration is maintained because of the state drugs induce, not because they alleviate an unpleasant state ${ }^{326,363,365}$.

But to state that addictive drugs are positive reinforcers does not explain addiction. As pointed out by Wise and Bozarth (ref. 365, p. 472): "To assert that all addictive drugs are reinforcers is to do little more than redefine the phenomenon of addiction."... "To identify a drug as reinforcing goes no further than to identify the drug as addicting, because it is the common observation of habitual self-administration that serves as the basis for most definitions of both drug reinforcement and drug addiction. A theory of addiction based on the concept of reinforcement would have to identify actions of drugs that are operationally independent of self-administration habits in order to offer insight as to why drugs are addictive." That is, positive reinforcement is merely a description of a behavioral effect, not an explanation of the effect ${ }^{307}$. The critical questions are, why are some drugs positively reinforcing (i.e., what specific actions of drugs are positively reinforcing) and why do drugs become more effective reinforcers as addiction develops? It is usually assumed that drugs act as positive reinforcers because they produce pleasure. Thus, Wise and Bozarth state ${ }^{365}$ "the only existing positive reinforcement view of addiction that might qualify as an explanatory theory identifies positive reinforcement with drug euphoria. In this view drugs are addicting (establish compulsive habits) because they produce euphoria or positive affect" (ref. 29 and ref. 213 , p. 474). Similarly, Stewart et al. ${ }^{326}$ argued that compulsive drug use is maintained by appetitive motivational states generated by the ability of drugs to produce positive affective statest.

There are, however, a number of problems with the hypothesis that the subjective pleasurable (hedonic) effects of drugs are either necessary or sufficient to motivate compulsive drug-seeking and drug-taking behavior. As put by Dews ${ }^{72}$ over 15 years ago, "it was supposed that the prediction of addiction liability was essentially equivalent to prediction of euphorigenic power. As with most self-evident ideas, the mere matter of there being essentially no evidence in favor of it and much against it, had little effect on its acceptance" (p. 75).

3.2.1. Problems with a positive reinforcement / euphoria view of addiction

- If the positive reinforcing effects of drugs are primarily due to their ability to produce pleasurable affective states (euphoriat) and if this is sufficient to produce addictive behavior, the subjective pleasurable effects of drugs must be enormous. Indeed, the subjective pleasurable effects of drugs would have to be so potent that just the memory of drug experiences would be sufficient to evoke compulsive drug-seeking and drug-taking behavior. Although addictive drugs can indeed produce extremely pleasant affective states (anonymous, personal communication) it is difficult to believe that this property of drugs alone is sufficient to account for addiction. For one, there is no clear relationship between the ability of individual drugs to produce 
euphoria and their addictive potential. For example, nicotine is considered highly addictive, but nicotine does not produce marked euphoria or other strong hedonic states (ref. 266 for example). Also, many addictive drugs can actually produce strong dysphoric states, especially with initial use. Second, it could be argued that in addicts the magnitude of the negative consequences of continued drug use often far outweigh the magnitude of drug pleasure or the memory of drug pleasure. In fact, to most people (including many addicts), the negative consequences of continued drug use, including loss of health, friends, family, home and job, seem enormous relative to the pleasure derived from drugs. Falk et al.$^{86}$ have pointed out (p. 58): "the apparent irrationality of these activities [drug use]. The activities seem to produce more harm than benefit for the individual. How could creatures have evolved such powerful, wasteful and even self-destructive propensities? Not only are the activities apparently irrational, but also an apparent disparity exists between the immediate consequences of the behavior and its strength. The rush of an intravenous injection is transient and with street-quality heroin, rarely dramatic. Yet, the drug somehow can support day-long hustling and determine a whole subculture".

Indeed, addicts will sometimes report that they are miserable, that their life is in ruins and that the drug is not even that great anymore - but they still want it! Addicts themselves often are bewildered by the intensity and irrationality of their own 'wanting'. It is difficult to explain this situation by just evoking short-lasting drug pleasure. If the incentive motivational effects of drugs are due only to their ability to produce transient pleasure, but the aversive consequences of continued drug use eventually come to far outweigh the pleasurable effects - and, if drug taking behavior is maintained by simple contingencies of reinforcement, self-administration behavior should extinguish. But it usually does not (although see Falk et al. ${ }^{86}$ for a discussion of the unique effects of intermittent schedules of reinforcement).

- A positive reinforcement/euphoria view of addiction does not adequately cxplain drug craving or relapse elicited by environmental stimuli associated with drug taking. Both Stewart et al. ${ }^{326}$ and Wise and Bozarth ${ }^{365}$ have argued convincingly that drug-related stimuli can evoke 'drug-like' effects that serve to motivate further drug-seeking and drug-taking behavior. They recently termed this view a 'proponent-process theory' ${ }^{329}$, in contrast with the 'opponent-process' view associated with negative reinforcement models ${ }^{182,312}$. Stewart and Wise ${ }^{329}$ argue "it is drug-like processes rather than drug-opposite processes that whet the appetite and stimulate renewed responding. In this view it is the 'taste' of the drug or the experience of stimuli that through Pavlovian conditioning - cause drug-like central effects that motivate drug intake in experienced subjects" (p. 80).

The question remains, however, what exactly is this 'drug-like process'? One possibility is that it resembles the positive affective state, the pleasurable state, evoked by the drug itself. That it is equivalent to what has been called a conditioned 'high' 50 . For example, Stewart et al. ${ }^{326}$ say: "Conditioned drug effects that mimic the unconditioned drug effects, as are conditioned positive affective states, are elicited by the environment where these drugs are experienced." (p. 264, our italics). In this view drug-associated stimuli may evoke 'conditioned pleasure', which reminds the addict of the even greater pleasure of the drug itself, thus motivating the individual to once again obtain the drug $^{362}$.

Addicts do report conditioned 'highs', as in the example of the oft cited 'needle freak'. In laboratory studies, however, self-reports of conditioned 'highs' occur much less frequently than self-reports of either conditioned craving or conditioned withdrawal-like signs $^{50,229}$. Self reports of conditioned craving are especially frequent. This suggests that conditioned craving is dissociable from conditioned 'highs' and therefore, in many instances, drug craving is not caused by a conditioned 'high'. How then is craving explained in the context of a positive reinforcement/euphoria view of addiction?

A second possibility is that relapse in a 'recovered' addict is triggered by cues that evoke an explicit memory or representation of past drug experiences. Unlike a 'conditioned high', which is accompanied by an affective experience similar to that produced by the drug itself, an explicit memory need not be pleasant in itself. It recalls past pleasure in a cognitive form, as a semantic proposition or as a conscious image of the act of drug taking and spurs the addict to attempt to regain the remembered experience of pleasure once again.

An 'explicit memory of past pleasure' interpretation of relapse is not implausible and may describe some instances of relapse. But an interpretation that posits explicit memories of taking drugs to be a sufficient cause for relapse finds it difficult to explain why relapse occurs only when it does. No addict who relapses after months or years of abstinence could possibly not have remembered drug experiences many times before. During the process of withdrawal every addict must often recall and dwell upon memories of the drug experience. Once withdrawal is successfully endured the circumstances of daily living would cause one to 
sometimes remember earlier times, when life was different and drugs were the focus. Even without any particular cue or reminder, the mere process of free association would often call to mind scenes from earlier life, including upon occasion, drug-related experiences. Why should the explicit memory of a drug experience suddenly be sufficient to trigger relapse, when a person has had innumerable previous memories of drug experiences without relapse?

An 'explicit memory of past pleasure' interpretation might reply that, because relapse is often triggered by particular situations that have been paired with drug use in the past, these situations evoke a memory that is more vivid than all the memories that have come before. Whether this is true or not is an empirical question. Certainly, vivid memories can be triggered by associative cues and there is ample evidence that associative context is a powerful modulator of conditioned behavior; although whether context modulates explicit memories in this way is less clear (ref. 259 for example). However, images or other forms of conscious remembering that occur during withdrawal, daily life or free association might also be expected to be fairly vivid at least some of the time. It is not intuitively obvious that these memories should necessarily differ in vividness or in any other subjective quality that might explain why some conscious memories provoke relapse when others do not. In other words, an 'explicit memory' hypothesis places an extraordinary explanatory burden on the crucial assumption that relapse-provoking memories are qualitatively different from the myriad other memories of drugs that do not provoke relapse. As far as we know, there is no evidence to support this assumption. - The most compelling evidence against the idea that drug taking is necessarily motivated by the subjective pleasurable effects of drugs comes from studies showing that drug self-administration can be maintained in the absence of subjective pleasure; that is, subjective pleasure is not necessary to maintain drug-seeking and drug-taking behavior. A striking example of a dissociation between the incentive motivational effects of morphine and the subjective pleasurable effects of morphine was reported recently by Lamb et al. ${ }^{192}$. These researchers found that opiate 'postaddicts' would work (press a lever) to get an injection of a low dose of morphine, despite the fact that four of five people could not distinguish the subjective effects of the morphine from the placebo - but the placebo did not reinforce lever pressing (ref. 151 as well). In other words, people 'self-administcred' a low dose of morphine and not the placebo, but reported that neither the drug nor the placebo produced pleasure; there was no subjective difference between them. Similar effects have been reported by Fischman and Foltin ${ }^{94,95}$ in laboratory studies of cocaine self-administration behavior in humans. Cocaine users reliably choose a low dose of cocaine ( $4 \mathrm{mg}$ ) over placebo, although this dose produces no self-reported subjective effects or cardiovascular effects. In addition, Fischman and Foltin ${ }^{95}$ report that within-session tolerance to many of the cardiovascular and subjective (euphoric) effects produced by higher doses of cocaine is not accompanied by changes in drug-taking behavior; that is, within a self-administration session a dissociation develops between the subjective effects of cocaine and cocaine self-administration behavior.

On the basis of their study Lamb et al. ${ }^{192}$ concluded "that the reinforcing effects of morphine can occur in the absence of self-reported subjective effects and thus, do not appear to be causually related to drug-liking or euphoria" (p. 1172, our italics). Similarly, when asked to speculate what maintains the self-administration of cocaine in the absence of subjective pleasure Fisch$\operatorname{man}^{95}$ replied:

"I think cocaine is maintaining their behavior! If you want to know what the subjects say about their self-administration of these low doses, they tell me that they were not choosing cocaine over placebo. They often insist that they were sampling equally from each of the two choice options and both were placebo. On the other hand, if you look at the data from that session, you see that they were choosing the low dose (as low as $4 \mathrm{mg}$ ) or the dose with no measurable effect."... "I do not believe that measuring subjective effects provides us with the information about 'what' is maintaining their cocaine-taking. The best we can say at this point is that it is the cocaine that is maintaining cocaine-taking" (p. 179).

Of course, these studies also suggest that a memory or representation of the subjective pleasurable effects of morphine or cocaine is not required to sustain drug-taking behavior either, because if that were true then there should be some subjective difference in memories during low-dose morphine or cocaine compared to the placebo and there was none. Clearly there is a difference between low-dose morphine or cocaine and the placebo, but the data suggest it is not a subjective difference; it is not explicit and does not have access to conscious awareness.

Although the effects described by Lamb et al. ${ }^{192}$ and Fischman and Foltin ${ }^{95}$ are particularly striking, dissociations between the subjective pleasurable effects of drugs and drug-taking behavior have been noted previously. For example, Falk et al ${ }^{86}$ review studies showing that "the subjective effects produced by a drug do not necessarily predict whether the drug actually will be 
self-administered" (p. 58). In addition, Katz and Goldberg ${ }^{165}$ describe experiments that suggest "the reinforcing effects and the subjective reports by human volunteers are not functionally equivalent entities" ( $p$. 24).

Similarly, studies in rats on the affective vs. reinforcing properties of opiates suggest these actions may be mediated by separable neural systems ${ }^{205,355}$. This was suggested, for example, by White et al. ${ }^{355}$ on the basis of studies on the effects of morphine in a runway task, in which food was available in the goal box. They found that morphine acted as a strong positive reinforcer, leading to faster and faster running speeds. But at the same time animals learned to avoid morphine-paired food. Thus, "the aversive effects of morphine were accompanied by positive reinforcement, a paradox that is difficult to understand" (ref. 355, p. 66). White et al. ${ }^{355}$ suggested an explanation for this paradox may be "that the reinforcing effects of morphine" do "not depend upon the affective properties of the drug, but that the drug directly activate(s) a neural mechanism of reinforcement, which facilitates learning independantly of the animal's affective state".

The studies cited above are very important because their findings directly contradict the central premise of a positive reinforcement/euphoria view of addiction. In our terms, they establish that the incentive motivational effects of drugs are not directly attributable to their subjective pleasurable effects: that is, drug 'wanting' is not equivalent to drug 'liking'.

In summary, both negative reinforcement (e.g., withdrawal avoidance) and positive reinforcement/ euphoria (pleasure-seeking) views of addiction have difficulty explaining a number of important features of addictive behavior. Any plausible new theory of addiction needs to address the same issues and better explain them. Specifically, an adequate theory of addiction must explain:

(1) What accounts for drug craving elicited by drug-associated stimuli, if craving is not causally related to conditioned withdrawal signs, conditioned 'highs' or the explicit memory of past pleasure?

(2) Why is craving sometimes highest immediately after drug administration, when subjective pleasurable effects are still predominant?

(3) Why does obsessive craving for drugs persist in the face of enormous negative consequences associated with continued drug use, and relatively modest subjective pleasurable effects?

(4) How can low doses of drugs, which do not produce discernible subjective pleasure or physical dependence, maintain drug-seeking and drug-taking behavior?

(5) Why is relapse such a prevalent and persistent feature of addiction, even in 'recovered' addicts?

(6) Why can relapse be precipitated by so many different stimuli (drugs, environmental stimuli associated with drugs, mood changes)?

\subsection{Requirements of an Incentive-Sensitization Theory of Addiction}

Neither traditional positive reinforcement nor negative reinforcement theories of addiction provide compelling answers to the questions listed above. We will argue below that the Incentive-Sensitization Theory of Addiction does. The Incentive-Sensitization Theory was introduced as a 'neuroadaptationist' model. It posits that repeated intermittent drug use results in incremental and persistent changes in a neural system that mediates craving for drugs; to be more precise, in a neural system responsible for the attribution of incentive salience (not pleasure) to stimuli. We first need to ask, therefore, whether there is any experimental evidence that repeated exposure to addictive drugs can produce neuroadaptations that meet the relevant critcria. The criteria required of such neuroadaptations in order for the theory to be true include the following:

(1) To the extent that the excessive incentive salience elicited by drugs is mediated by a common substrate, there should be a common neural system affected by many different addictive drugs.

(2) To explain the progressive development of addictive behavior repeated drug administration should render this neural system hypersensitive in a gradual and incremental fashion.

(3) To explain the persistence of relapse these drug-induced neuroadaptations should persist for very long periods of time (if not permanently) following the discontinuation of drug use.

(4) To explain the role of drug-associated stimuli in relapse the expression of these neuroadaptations should be susceptible to conditioned stimulus or environmental control.

(5) To explain drug craving the activation of this neural system should mediate the incentive motivational effects of drugs and drug-related stimuli and the neuroadaptations produced by drugs should potentiate these motivational effects.

(6) To explain the dissociation between drug 'wanting' and drug 'liking' this neural system should not directly mediate the subjective pleasurable effects of drugs or the subjective pleasure associated with other stimuli.

Evidence that the repeated use of a number of different addictive drugs does indeed produce neuroadaptations that meet each of these criteria is presented next. 


\section{EVIDENCE FOR THE INCENTIVE-SENSITIZATION THEORY OF ADDICTION}

\subsection{Criterion 1: there should be a common neural system affected by many different addictive drugs}

Addictive drugs represent a diverse group of compounds that markedly differ in their behavioral and neurochemical actions. Nevertheless, there is increasing evidence that many addictive drugs share the ability to enhance mesotelencephalic dopamine neurotransmission ${ }^{73,365}$. This evidence has been reviewed recently and need not be reiterated here. Suffice it to say that the following drugs have been reported to increase dopamine neurotransmission in the nucleus accumbens and dorsal striatum: amphetamine ('speed'), cathinone ('Khat'), cocaine ('coke'; 'crack'), methamphetamine ('ice'; 'crystal meth'), methylenedioxyamphetamine (MDA; 'the love drug'); methylenedioxymethamphetamine (MDMA; 'ecstasy'), methylphenidate, ethanol, fentanyl ('China white'), methadone, morphine, nicotine and phencyclidine (PCP; 'angel dust'). This common action of diverse drugs is consistent with the hypothesis that mesotelencephalic dopamine systems mediate, at least in part, the incentive motivational properties of many different drugs of abuse ${ }^{362,363}$. Although it cannot be said that there is a single neural system that is affected by all addictive drugs, dopamine systems and their associated structures are affected by most $^{179,363,365}$.

\subsection{Criterion 2: the repeated administration of different} addictive drugs should render a common neural system hypersensitive in a gradual and incremental fashion

Drug effects are known to change when drugs are given repeatedly, and some of these changes are known involve adaptations in neural elements mediating specific drug effects ${ }^{148}$. Much of the emphasis in the past has been on homeostatic neuroadaptations thought to underly the development of tolerance and to contribute to withdrawal symptoms. But as discussed above, tolerance and withdrawal do not account for the defining characteristics of addiction and therefore, tolerance and withdrawal-associated neuroadaptations do not meet Criterion 2 under discussion here.

4.2.1. Sensitization to the psychomotor-activating effects of addictive drugs. However, some effects of drugs are not decreased, but are actually increased by repeated intermittent drug administration. Indeed, for a given drug, some effects may decrease (show tolerance) while simultaneously other effects increase. This latter phenumenon has been referred to as behavioral sensitization, behavioral facilitation, reverse tolerance and auxoesthesia ${ }^{160,162,201,257,272,291,297,354}$. We will use the term 'sensitization' here.

Psychomotor stimulant drugs, such as amphetamine or cocaine, have been used in the majority of studies on drug-induced sensitization, and the effects of these drugs have been well characterized ${ }^{188,250,257,269,272,291,354}$. For example, the acute administration of a low-tomoderate dose of amphetamine or cocaine produces 'psychomotor activation', characterized by an increasc in locomotor activity (ambulation), rearing behavior and rotational behavior ${ }^{267}$. Higher doses result in the emergence of focussed stereotyped behaviors, such as repetitive head movements and sniffing and a resultant decrease in locomotion and rearing 254,284 . The repeated intermittent administration of a constant, relatively low dose, produces a progressive increase in drug-induced locomotor stimulation with each successive administration. Repeated administration of a moderate dose will come to elicit the stereotyped behavior typical of a higher dose, even though it produced only ambulation the first time it was given. Furthermore, sensitization-related changes in behavior can come under strong conditioned stimulus control and this feature of sensitization is discussed in more detail below. In summary, it is the gradual and incremental increase in drug-induced 'psychomotor activation' and the emergence of increasingly stereotyped behavior, that is usually referred to as 'behavioral sensitization'. The only comparable stimulant effect that has been characterized in humans is sensitization to the psychotogenic effects of amphetamine and cocaine $250,272,280,297$.

Behavioral sensitization is produced by the repeated administration of many different psychomotor stimulants, including the amphetamines ${ }^{8,188,272,292}$, cocaine $^{160,250}$, methylphenidate ${ }^{40,177,301}$, fencamfamine ${ }^{3}$ and the endogenous trace amine, phenylethylamine ${ }^{35}$. The phenomenon is not limited, however, to classical psychomotor stimulants. Other drugs, not traditionally considered psychomotor stimulants, also produce psychomotor activation, enhance dopamine neurotransmission and produce behavioral sensitization ${ }^{73,365}$. These include: opioids ${ }^{11,153,302}$, nicotine 25,52,171,186, $^{2}$ phencyclidine ${ }^{116,117,147,225}$, ethanol ${ }^{58,61,114,208,209}$ and MDMA $^{316}$ (cf. Note 7 in Ch. 6).

Repeated intermittent treatment with an addictive drug not only produces sensitization to that drug, but may also produce cross-sensitization to other drugs. Although the literature is not entirely consistent, cross-sensitization has been reported between drugs in the same class (e.g., amphetamine and cocaine) and between drugs in different classes (e.g., stimulants and opioids) ${ }^{159.160 .268}$. Furthermore, cross-sensitization is also found between drugs and stress, which led to the 
suggestion that drugs may induce sensitization by their action as stressors ${ }^{8,9,249}$. Evidence for cross-sensitization between drugs and stress comes from studies showing that animals pretreated with drugs like amphetamine, cocaine or morphine are later hyperresponsive to stress and vice versa, animals sensitized to stress are hyperresponsive to a drug challenge $e^{8,9,160,190,196,268}$. Even the repeated administration of exogenous corticosterone is reported to increase the locomotor response to a later challenge with amphetamine ${ }^{70}$ (see also Note 9 in Ch. 6).

4.2.2. Sensitization to the incentive motivational effects of drugs. Of more direct relevance to the process of addiction are recent studies on sensitization to the incentive motivational properties of addictive drugs. There has been relatively little research on this topic, but there have been a number of recent experiments, using either self-administration procedures or the conditioned place preference paradigm, which suggest that prior exposure to amphetamine, cocaine or morphine produces sensitization to the incentive motivational effects of these drugs.

In one of the first reports of this kind Woolverton et al. ${ }^{373}$ found that rhesus monkeys would self-administer a low dose of methamphetamine only after they had received a regimen of non-contingent injections of methamphetamine. That is, the threshold dose necessary to sustain self-administration was lowered by methamphetamine pretreatment, suggesting "an increased sensitivity to the reinforcing properties of the drug" (p. 740). There are now a number of similar reports in rats ${ }^{241}$. For example, Piazza and his coworkers ${ }^{242,243}$ have reported that $d$-amphetamine pretreatment, which induces behavioral sensitization, facilitates the later acquisition of an amphetamine self-administration habit, especially in animals not predisposed to self-administer amphetamine. Similarly, pretreatment with cocaine facilitates the later acquisition of a cocaine self-administration habit ${ }^{137}$.

Sensitization has also been observed with the conditioned place preference paradigm, in which the place where a drug is experienced becomes preferred by an animal in subsequent choice tests. For example, Lett ${ }^{195}$ examined the influence of amphetamine, cocaine or morphine pretreatment on the later acquisition of a conditioned place preference produced by the same drug. For all three drugs, pretreated (sensitized) animals showed a significantly enhanced conditioned place preference, relative to control animals. A similar effect has been reported following pretreatment with morphine $^{102}$ or ethanol ${ }^{114}$.

Cross-sensitization also occurs in these situations. Animals pretreated with amphetamine show an en- hanced place preference for morphine, animals pretreated with morphine show an enhanced place preference for cocaine and animals pretreated with morphine show an enhanced place preference for amphetamine ${ }^{195}$. These latter findings suggest that the enhancement of the conditioned place preference is not due to tolerance to the drug's aversive properties, because cross-tolerance does not occur between the stimulants and morphine ${ }^{195}$. Similarly, cross-sensitization has been found using self-administration procedures. Noncontingent pretreatment with amphetamine, caffeine or nicotine facilitates the later acquisition of cocaine self-administration ${ }^{136-138,282}$. An especially intriguing example of cross-sensitization between opiates and amphetamine was reported recently by Cunningham and Kelley ${ }^{62}$. These researchers found that the repeated intra-accumbens application of a mu receptor agonist (morphine or DAMGO) for 4 days potentiated (sensitized) the ability of systemic amphetamine to later enhance responding for a conditioned reinforcer; i.e., a light/tone previously paired with food.

It was mentioned above that cross-sensitization to the psychomotor-activating effects of drugs can occur not only between drugs, but between drugs and stress. Therefore, the effects of prior stress on the incentive motivational effects of drugs are also of interest. Indeed, prior stress (tail pinch) facilitates the acquisition of an amphetamine self-administration habit ${ }^{243}$. In fact, a number of potentially stressful environmental manipulations, such as social isolation or prenatal stress, are reported to increase sensitivity to amphetamine and facilitate amphetamine or cocaine self-administration behavior ${ }^{68,152,281}$.

In conclusion, these studies establish that not only are the 'psychomotor-activating' properties of addictive drugs sensitized by repeated drug administration, but their incentive motivational properties are sensitized as well. Animals sensitized to amphetamine, cocaine or morphine later show an enhanced preference for an environment associated with drug administration and animals sensitized to amphetamine or cocaine show enhanced vulnerability to acquire a drug self-administration habit. Obviously, sensitization to the incentive motivational properties of drugs (and drug-related stimuli) could have a profound influence on the development of addictive behavior. With more-and-more drug experience the incentive value of the act of drugtaking and of drug-related stimuli would be progressively enhanced, which would increase the probability of repeating drug-seeking and drug-taking behavior in the future (although, see Note 1 in Ch. 6 for a discussion of the role of response contingency in drug sensitization). 
4.2.3. The neural basis of behavioral sensitization. The behavioral studies summarized above strongly suggest that the repeated administration of many different addictive drugs produces gradual and incremental neuroadaptations that render animals hypersensitive to these agents. The behavioral studies also provide prima facie evidence that sensitization-related neuroadaptations involve a hypersensitivity in mesotelencephalic dopamine systems. A change in dopamine neurotransmission is implicated for a number of reasons. First, the behaviors that are sensitized by addictive drugs are known to involve an activation of mesotelencephalic dopamine systems. There is considerable evidence that both the psychomotor-activating effects and the incentive motivational effects of many of these drugs requires the integrity of mesotelencephalic dopamine systems, especially dopamine projections to the ventral striatum $^{362}$. Second, the activation of dopamine systems appears to be necessary to induce sensitization. The sensitization produced by amphetamine, cocaine or morphine is prevented by co-treatment with dopamine antagonists ${ }^{121,187,344,345,352}$ (see ref. 160 for a review) and amphetamine sensitization is prevented by a 6-OHDA lesion ${ }^{293}$. Third, the application of amphetamine or morphine directly into the ventral tegmental area, where dopamine cell bodies are located, induces sensitization ${ }^{160}$. Fourth, a local 'challenge' injection of amphctamine into the lateral ventricle ${ }^{258}$ or nucleus accumbens ${ }^{178,233}$ evokes a sensitized behavioral response in animals treated previously with systemic amphetamine.

Perhaps even more importantly, behavioral sensitization is accompanied by changes in mesotelencephalic dopamine activity $160,257,269,2 / 2,354$. For example, amphetamine sensitization is accompanied by an increase in amphetamine-stimulated dopamine release from striatal tissue in vitro ${ }^{48,176,178,271,358,375}$. More recently, in vivo microdialysis studies have shown that although amphetamine sensitization is not accompanied by changes in the basal extracellular concentration of dopamine, it is associated with an enhanced dopamine response to a drug challenge $\mathrm{e}^{14,166,231,269,274,370}$ (cf. ref. 295 ). Even the local application of amphetamine into the ventral tegmental area sensitizes the dopamine release produced by a subsequent systemic challenge with amphetamine ${ }^{347}$. A similar enhancement in dopamine response has also been reported in associa-

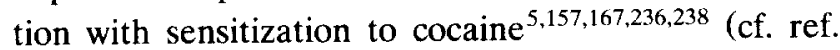
296), ethanol ${ }^{24}$, nicotine ${ }^{25,101.123}$ (cf. ref. 65), morphine ${ }^{154,160}$ (cf. ref. 155), phenylethylamine ${ }^{189}$ and methylphenidate ${ }^{177}$ and following cross-sensitization between different drugs $s^{4,159,166}$ and between drugs and stress ${ }^{156,159,160,315,320,358}$. Furthermore, co-treatment with a dopamine receptor antagonist, which prevents the induction of behavioral sensitization to methamphetamine, also attenuates the dopaminergic response to a methamphetamine challenge assessed with microdialysis ${ }^{121}$. Although there is no convincing evidence for sensitization-related changes in dopamine receptor binding ${ }^{272}$, there is electrophysiological evidence for an increased sensitivity of nucleus accumbens neurons to iontophoretically applied dopamine in cocaine sensitized rats ${ }^{124,354}$. It is possible, therefore, that sensitization produced by cocaine and amphetamine is also accompanied by changes in the transduction of dopamine receptor-mediated events ${ }^{15,112,124,140,265,276}$.

The biophysical basis of sensitization-related changes in dopamine neurotransmission is not known. There have been a number of hypotheses proposed, including changes in autoreceptor sensitivity, changes in the intraneuronal distribution of dopamine leading to enhanced release, changes in the dopamine uptake carrier and changes in transduction mechanisms ${ }^{6,160,188,226,269,354}$. It is not known, however, whether the behavioral sensitization produced by different drugs involves the same or different cellular and molecular changes. Even the processes involved in the induction of sensitization differ from those involved in the expression of sensitization ${ }^{160,233,269}$. Furthermore, sensitization-related changes in dopamine systems have been emphasized here because only dopamine systems have been studied in any detail. But neuroadaptations in other neurotransmitter systems that intcract with dopamine systems must also be considered. For example, glutamate systems have been implicated in recent studies showing that glutamate antagonists, like dopamine antagonists, prevent the induction of sensitization $^{161,163,164,369}$.

In summary, there is considerable experimental evidence in support of Criterion 2. The repeated administration of many different addictive drugs produces behavioral sensitization and behavioral sensitization is associated with hypersensitive mesotelencephalic dopamine systems.

\subsection{Criterion 3: sensitization-related neuroadaptations should be very long-lasting}

One of the most striking characteristics of sensitization is its persistence. A single injection of amphetamine, cocaine or morphine induces behavioral sensitization lasting for weeks to months ${ }^{40,197,236,237,267,302}$ and animals sensitized with escalating doses of amphetamine remain behaviorally hypersensitive to an amphetamine challenge for at least 1 year ${ }^{232}$. In fact, Paulson et al. ${ }^{232}$ found that rats were just as sensitized a year following the discontinua- 
tion of amphetamine pretreatment, which is over one third of their life-span, as they were at 2-4 weeks. These findings suggest that after at least some pretreatment regimens the neuroadaptations responsible for behavioral sensitization to amphetamine may be essentially permanent. Similarly, behavioral sensitization in rats is reported to persist for over 3 months following pretreatment with cocaine ${ }^{304}$, for over 50 days following pretreatment with methylphenidate ${ }^{301}$ and for over 8 months following pretreatment with morphine ${ }^{12,17,302}$.

It is not known if sensitization to the incentive motivational properties of drugs persists for as long as sensitization to their psychomotor-activating effects, but this is obviously an important issue for the Incentive-Sensitization Theory. To the extent that sensitization to the psychomotor-activating effects and the incentive motivational effects of drugs have a common neural basis we would expect both effects to show comparable persistence. Neither have neurochemical studies been conducted as long as a year following drug pretreatment. But sensitization-related changes in dopamine systems have been reported to persist for months after withdrawal ${ }^{269,272}$. The available evidence suggests, therefore, that the neuroadaptations underlying behavioral sensitization meet the criterion of persistence.

4.4. Criterion 4: the expression of sensitization-related neuroadaptations should be amenable to conditioned stimulus control

The Incentive-Sensitization Theory of Addiction posits that drugs sensitize a neural system that mediates 'wanting'. It is also hypothesized that associative processes focus exaggerated 'wanting' (craving) specifically onto drug-related stimuli. This implies that the behavioral expression of sensitization-related neuroadaptations should be strongly influenced by associative factors.

Indeed, the environmental context in which drugs are experienced can have profound effects on the development and expression of sensitization. Stewart ${ }^{323}$ recently reviewed the literature on the conditioned stimulus control of sensitization and the reader is referred to this paper for a more detailed and excellent discussion of such issues. In brief, Stewart ${ }^{323}$ points out that the conditioned stimulus control of sensitization can take one of two basic forms, depending to some extent on experimental design. In one design the drug (the UCS), which produces a pharmacological effect (the UCR), is given only in association with unique environmental cues (CS). After repeated pairing of the UCS and CS, the CS alone can acquire the ability to elicit drug-like responses (CR). For example, after repeated administration of a dose of amphetamine that produces locomotor hyperactivity, just placing an animal in the environment in which it previously received amphetamine is sufficient to produce conditioned locomotor hyperactivity, in the absence of any drug. A number of researchers have reported drug-environment conditioning of this type and have suggested these conditioned effects contribute to the development of sensitization ${ }^{128,283,323}$ (see ref. 338 for a review).

A second type of conditioned stimulus control of sensitization is the situation where, after explicit pairing of a drug and specific test environment, animals are administered a 'challenge' injection of the drug in either the drug-paired environment or in a new environment. In this case one observes the effect of the CS (the environment) on the response to the UCS (the drug). In some experiments of this type the expression of sensitization has come under complete conditioned stimulus control. For example, Post et al. ${ }^{251}$ reported that rats given ten daily injections of $10 \mathrm{mg} / \mathrm{kg}$ of cocaine in a test environment showed a progressively greater behavioral response (locomotor activation) to the drug, but animals given daily injections of cocaine in their home cage did not show evidence of behavioral sensitization when subsequently challenged with cocaine in the test environment. Similarly, Vezina and Stewart ${ }^{344}$ reported that repeated injections of morphine into the ventral tegmental area produced evidence of sensitization on a subsequent challenge test only when rats were tested in the environment where they received morphine. In addition, the conditioned stimulus control of sensitization may not only enhance the expression of sensitization in drug-paired environments, but may also inhibit the expression of sensitization in environments that predict the absence of the drug. For example, Vezina and Stewart ${ }^{344}$ found that the locomotor response to an intra-ventral tegmental area challenge injection of morphine in explicitly unpaired animals was significantly depressed relative to saline-pretreated controls (also see ref. 328). Nevertheless, sensitization is not only a conditioned response, even though the expression of sensitization can come under strong conditioned stimulus control, a point that has been made by a number of authors ${ }^{40,210,272,323,346}$.

Our discussion of the conditioned stimulus control of sensitization has focused thus far on the effects of drug-associated stimuli on the subsequent response to drugs. It is important to know, however, whether environmental stimuli associated with drugs can also influence the response to other incentive stimuli; even non-drug-related stimuli. This issue has received al- 
most no experimental attention, despite its theoretical importance. Nevertheless, it was addressed in one study by Mitchell and Stewart ${ }^{218}$. In this study male rats were given morphine either in a test arena or their home cage, every other day for four injections. All rats were then placed in the test arena in the presence of a receptive female. Rats pretreated with morphine in the test arena showed more frequent female-directed behaviors than either rats pretreated with morphine in their home cage or saline-pretreated controls. The enhancement of sexual behavior produced by the drug-associated environment did not involve changes in copulatory behavior per se, but only in the appetitive behaviors leading to copulation, including more frequent "pursuit of the female, anogenital exploration and partial mounts and... shorter latencies to initiate copulation" (p. 367). That is, the female appeared to be a more salient incentive stimulus in male rats tested in the presence of morphine-associated cues. These results suggest that sensitization to drugs may change neural systems that not only modulate the incentive properties of drug-associated stimuli, but the incentive properties of "natural incentives't. This is important because it implies that drug-associated stimuli may potentially influence a wide range of motivated behaviors.

To summarize thus far, we have addressed the first four criteria, and reviewed evidence to establish that: (1) Many different addictive drugs activate a common neural system, the mesotelencephalic dopamine system; (2) Repeated administration of many addictive drugs causes dopamine systems to become hypersensitive and this is accompanied by a gradual and incremental increase (sensitization) in the psychomotoractivating and incentive motivational properties of drugs; (3) The neuroadaptations underlying sensitization are extremely persistent; and (4) The expression of sensitization is subject to conditioned stimulus control.

We next need to address Criteria 5 and 6. Criterion 5 requires that the neural system sensitized by repeated treatment with addictive drugs normally mediate the incentive motivational effects of drugs and drug-relatcd stimuli. Criterion 6 requires that this neural system not mediate the subjective pleasurable effects of drugs or the pleasure associated with other stimuli.

\subsection{Criterion 5: the role of mesotelencephalic dopamine systems in incentive motivation}

There is a wealth of evidence implicating dopamine systems in the incentive motivational $\dagger$ effects of drugs, as well as of food, sex and other natural incentives $^{19,84,90,131,262,263,356,365,368}$ (cf. ref. 181). For example, signals predicting the availability of food, water or a sexual partner activate brain dopamine systems, increasing dopamine neurotransmission in the ventral striatum $^{51,66,126,246,376}$. Addictive drugs also increase dopamine neurotransmission ${ }^{73}$. Indeed, in the case of drugs, a direct action on dopamine systems alone is sufficient to motivate behavior. Animals will work for microinjections of drugs directly into appropriate portions of the mesotelencephalic dopamine system ${ }^{366}$. Furthermore, microinjections of amphetamine or dopamine directly into the nucleus accumbens facilitates responding for conditioned incentive stimuli $\dagger$ (conditioned reinforcers) - stimuli that have acquired incentive properties by association with a natural incentive ${ }^{\dagger 85,262,263}$. In short, a common neural currency for many incentives appears to be activation of mesotelencephalic dopamine systems (see Note 4 in Ch. 6).

Consistent with this idea are studies showing that the motivational properties of natural incentives and addictive drugs are attenuated by decreasing dopamine activity ${ }^{360}$. Antagonist drugs that prevent the activation of dopamine receptors or the complete destruction of mesotelencephalic dopamine projections by neurochemically selective toxins, impair the instrumental performance of animals for food, for drugs and for electrical brain stimulation. A great deal of effort has been directed towards ascertaining whether the suppression of dopamine neurotransmission produces changes in behavior because of effects on the control of movement or because of effects on incentive motivation and a variety of experimental paradigms have been developed to distinguish between motor and motivational effects (see Note 2 in Ch. 6). It is now generally accepted that dopamine antagonists can have effects on behavior that are truly motivational. This is not to say they may not also have effects that are 'motor', but in many cases the effect on behavior is precisely what one would expect if dopamine antagonism reduced the motivational properties of incentives ${ }^{82,360,361}$.

In summary, the large literature on the role of dopamine in mediating the incentive motivational effects of drugs and other stimuli satisfies Criterion 5. What of Criterion 6, which requires that dopamine not mediate the subjective pleasurable effects of drugs or the pleasure associated with natural incentives.

\subsection{Criterion 6: the effects of dopamine are on incentive salience, not pleasure}

The evidence that brain dopaminc systems mediate the incentive motivational effects of natural incentives and addictive drugs provided the basis for Wise's ${ }^{359,360,361}$ provocative anhedonia hypothesis: the hypothesis that mesotelencephalic dopamine systems 
mediate the subjective pleasure produced by food, drugs, electrical brain stimulation, etc. and that dopamine antagonists suppress the pleasure produced by these agents. The anhedonia hypothesis provided a parsimonious explanation of dopamine's motivational effects by equating it with the psychological process of subjective pleasure *.

If the motivational properties of natural incentive stimuli or drugs depended only on their ability to produce subjective pleasure, then the effects of dopamine manipulations on motivated behavior would be compelling evidence for the anhedonia hypothesis. We suggest, however, that incentive motivation depends on a number of additional psychological processes that interact with pleasure: including associative learning and the attribution of incentive salience to external events and their representations ${ }^{27,28}$. It is the entire complex of pleasure, learning and incentive salience $\dagger$ together that comprise the process of incentive motivation $\dagger$.

4.6.1. Incentive salience. Of particular importance to the Incentive-Sensitization Theory of Addiction are the relative roles of 'wanting' and 'liking' in incentive motivation. The idea that there may be a psychological process (and neural substrate) responsible for 'wanting' incentives that is dissociable from the psychological process (and neural substrate) responsible for 'liking' incentives has not been considered explicitly in previous theories of incentive motivation ${ }^{30,339, * *}$. Based on a series of studies on the relationship between taste pleasure and appetite, such a dissociation was recently proposed $^{27,28}$. Berridge and his colleagues hypothesized that a psychological process specifically involving the attribution of salience to incentive stimuli (incentive salience) results in the experience of 'wanting'. This view of incentive motivation posits that salience attribution is a specific psychological process that is activated normally in conjunction with pleasure ("liking') and associative learning in the creation of new incentives. As new incentives are acquired particular stimuli that allow an individual to recognize an incentive (e.g., the sight of food; in nature, a flowering plant that signals the availability of food; or, in the laboratory, a tone that predicts food), become associated with the pleasure food produces by the process of classical conditioning. The stimulus features of the incentive predict the pleasure that will follow and may elicit conditioned pleasure. But conditioned stimulus features also become themselves the target of a separate and powerful motivational process - salience attribution. Salience attribution transforms the sensory features of the incentive stimulus into an especially salient percept, which 'grabs attention', becomes attractive and 'wanted' and thus guides behavior to the incentive. That is, new incentives become attractive in their own right as conditioned incentives $\uparrow$ (also called conditioned or secondary reinforcers).

Thus, the role of salience attribution in incentive motivation is proposed to occur as the third stage of a three-stage process (see Fig. 2). First, pleasure is normally activated by an encounter with a natural incentive, such as when an hungry animal eats food. In the normal course of events, pleasure is a necessary step in the establishment of a new conditioned incentive. However, pleasure ('liking') is not by itself sufficient to motivate behavior ${ }^{28}$. Pleasure by itself has no object or action. Assignment of pleasure to something requires associative learning, which is the second stage in the formation of incentives.

If pleasure is assigned to an action or stimulus by associative learning, then the action or stimulus should come to predict pleasure or elicit pleasure, on its own. No doubt this often happens. But we would argue that neither the experience of pleasure nor the expectation of impending pleasure by themselves constitute 'wanting ${ }^{27,28}$. 'Wanting' requires an additional process: the attribution of incentive salience to stimuli or actions. Stimuli that are attributed with incentive salience become attractive and demand attention. Like the sight of food to a starving person, they cannot be ignored. This does not necessarily make them 'liked'; the sight of food may be irresistibly attractive to the starving person, but if out of reach it may torment rather than please. But the food is still much 'wanted'. In summary, incentive motivation is proposed to involve three distinct psychological processes acting together; plcasure, associative learning and incentive salience and different neural systems are thought to be responsible for $\operatorname{each}^{27,28, * * *}$.

Changes in any one of these three processes that

\footnotetext{
* Note, however, that in discussing his anhedonia hypothesis of dopamine blockade Wise ${ }^{361}$ stated: "The anhedonia hypothesis is most vulnerable in its assumption that positive hedonic states such as pleasure or euphoria are attenuated by neuroleptics. This is, for the most part, speculation" (p. 184).

** Although neither Bindra nor Toates distinguished 'wanting' from 'liking', Toates ${ }^{339}$ did distinguish the associative control of 'wanting/liking' from mere recall of past 'wanting/liking'. For example, as in the Krieckhaus effect, a stimulus that is ordinarily not 'wanted' or 'liked' may suddenly become 'wanted/liked' on the basis of its prior associations when physiological state is changed ${ }^{185}$.

*** For a discussion of evidence that most motivated behavior is primarily controlled by incentive processes see Toates ${ }^{339}$.
} 
constitute incentive motivation would produce effects on instrumental or appetitive behavior indistinguishable, by most measures, from changes in another. Mesotelencephalic dopamine does not seem to be critical specifically for associative learning; i.e., for forming stimulus-stimulus associations ${ }^{19.90}$. For example, dopamine antagonists do not disrupt the learning of associations between a stimulus and electrical shock, as measured by a conditioned emotional response or by the defensive burying paradigm ${ }^{22,23,141}$. It is more likely, therefore, that the effects of dopamine on motivated behavior are due to effects on subjective pleasure or incentive salience. If mesotelencephalic dopamine systems mediate the subjective pleasurable effects of drugs, then the addictive property of drugs such as cocaine, heroin, amphetamine, etc., which sensitize dopamine systems, might be best understood in terms of the pleasure these drugs induce. But if a sensitized dopamine system enhanced the incentive salience of the act of taking drugs, rather than the subjective pleasurable effects of drugs, then the neuroadaptations underlying sensitization would cause drugs to be 'wanted' or craved, independently of the pleasure they produce.

Most animal studies on the effects of dopamine antagonists on motivated behavior (summarized in Note 2 in Ch. 6) are equally consistent with the hypothesis that dopamine mediates pleasure (the anhedonia hypothesis of Wise) and the hypothesis that dopamine mediates incentive salience ${ }^{27,28}$. Likewise, studies on the effects of manipulating dopamine systems on the incentive properties of conditioned reinforcers are consistent with both interpretations of dopamine function ${ }^{85,262}$. In these latter experiments animals must learn a new instrumental response (bar press) that is reinforced only by a stimulus (light/tone) that was previously associated with a primary incentive, such as food, water or a mate. Manipulations that increase dopamine neurotransmission in the ventral striatum potentiate the incentive properties of conditioned reinforcers and manipulations that decrease dopamine neurotransmission in the ventral striatum block these potentiating effects $44,127,168,169,264,333,334$. Barry Everitt has suggested these studies support a view that, in some way "ventral striatal dopamine makes the world brighter" (Catecholamine Symposium, Amsterdam, 1992). This view is compatible with the attribution of incentive salience hypothesis proposed here. Nevertheless, these studies could also be interpreted in support of the 'pleasure' hypothesis and therefore, they do not resolve the issue.

4.6.2. Introspection into 'wanting' and 'liking'. At first glance it seems it should be relatively easy to resolve the issue by asking people to report what effect dopamine antagonist drugs have on the subjective effects of addictive drugs. Indeed, Wise ${ }^{361}$ challenged researchers to test this hypothesis, arguing that "it would be procedurally simple for workers using neuroleptics with human subjects to determine the effects of these drugs on the subjective effect of rewarding stimuli". Despite his challenge, the relationship between dopamine blockade and anhedonia remains equivocal and the data are inconsistent ${ }^{48,298}$.

In light of the incentive salience hypothesis, however, assessment of the effects of dopamine blockade on subjective pleasure in humans may not be as simple as Wise thought. In fact, it may be an especially difficult task ${ }^{228}$. Humans may not, under normal conditions, be able to subjectively tell the difference between the two psychological processes of 'wanting' versus 'liking'. Studies of introspection and self-report have established that humans often (a) report psychological 'events' that can be shown to have not happened; (b) strongly deny the existence of psychological events that can be shown to have influenced their behavior; and (c) confuse events that are not connected ${ }^{228}$. Under many circumstances humans actually have very little direct access into the nature of their own psychological processes. Rather, introspection appears to interpret underlying processes in ways that seem plausible to the person ${ }^{228}$. Introspection does not reveal psychological processes directly. This implies that a person might mistake a change in incentive salience for a change in pleasure ('If I don't want it, then I must not like it'). It may be possible to distinguish between changes in 'wanting' and 'liking' by asking appropriate questions that tap into different aspects of a person's reaction to an incentive, but this will have to be approached with great sophistication and caution.

4.6.3. Evidence that mesotelencephalic dopamine mediates incentive salience, not pleasure. Although the studies cited above are equivocal, there are at least four lines of evidence which lead us to suggest that sensitization of dopamine neurotransmission produces enhanced incentive salience rather than enhanced pleasure. The evidence is based both on reports by human addicts of their own experience and on studies in animals on the role of dopamine in sensory pleasure and motivated behavior.

- The first line of evidence comes from animal studies that have explicitly examined the role of dopamine in mediating natural reactions to the sensory qualities of tastes. These studies have used the 'taste reactivity paradigm', which is based upon natural hedonic and aversive reactions (tongue protrusions, gapes, forelimb 

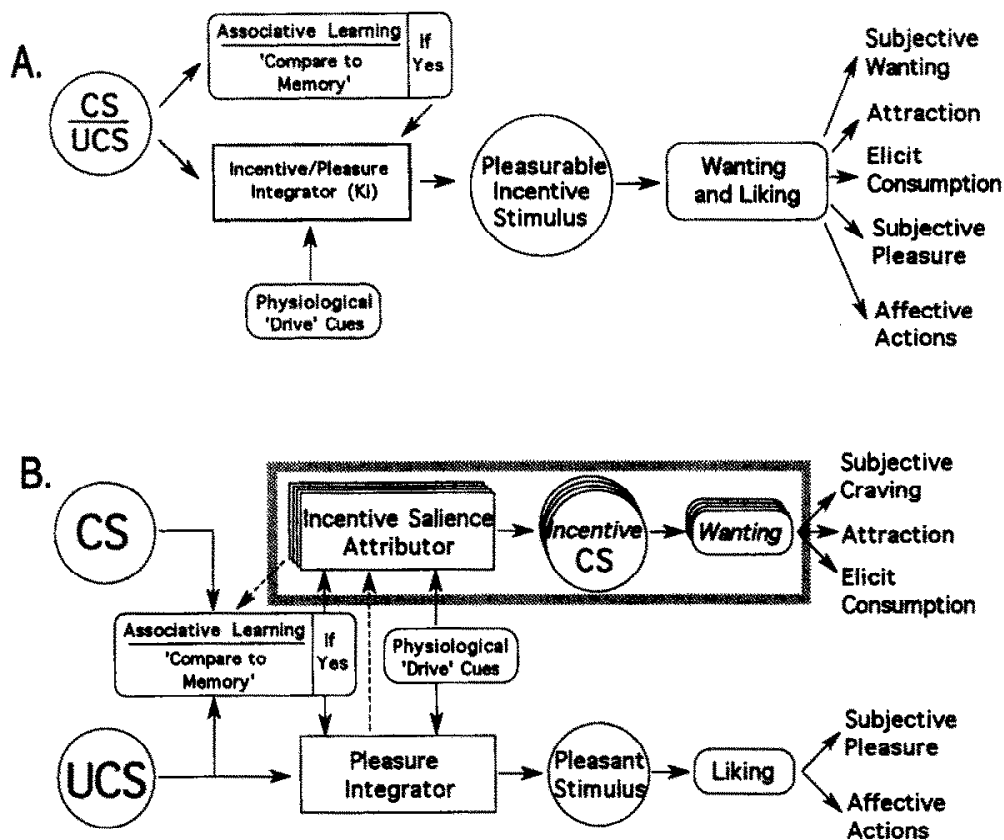

Fig. 2. A schematic illustration of a major existing model of the psychological processes that constitute incentive motivation (A, top) and our alternative model, which proposes a separate process of incentive salience and accounts for the consequences of drug-induced sensitization (B, bottom). Panel A: the 'Toates/Bindra model' of incentive motivation, on which our model is based (adapted from Toates ${ }^{339}$ ). By this model the sensory stimuli (CS and UCS) of incentive objects are both pleasant and attractive. Their ability to produce an incentive motivational state $\left(\mathrm{K}_{\mathrm{i}}\right)$ is partly dependent on memories of previous favorable experiences with them. Physiological states (such as hunger, thirst or withdrawal) selectively potentiate the ability of particular stimuli (related to food, water or drugs) to evoke incentive processes: to become 'wanted' and 'liked' (see Toates $^{339}$ for a more complete description of this model). Panel B: in our modified model of incentive motivation the psychological process (and neural substrate) for pleasure ('liking') is separate from the psychological process (and neural substrate) responsible for incentive salience ('wanting'). We further propose that the activation of mesotelencephalic dopamine systems plays a direct role only in the process of 'wanting', via the attribution of incentive salience to the perception and representation of conditioned stimuli (as described by Berridge and Valenstein ${ }^{27}$; also see Note 4 in $\mathrm{Ch} .6$ ). In Panel B the portion of the model (i.e., the psychological process) that is sensitized by repeated drug administration is 'highlighted' within the shaded box. It is the hyperactivation of this specific psychological process (incentive salience), due to sensitization of its neural substrate by drugs, that results in the excessive attribution of incentive salience to drug-related stimuli. Whereas normal levels of incentive salience attribution results in normal 'wanting', we propose hyperactivation of this system results in excessive incentive salience attribution, which is experienced as craving. Craving is pathologically intense 'wanting'. The major difference between our model of incentive motivation and the traditional model is that in ours the psychological processes and neural substrates responsible for pleasure ('liking') are separate from those for incentive salience ('wanting'). Thus, in our model 'natural incentives' (UCS stimuli) produce pleasure directly, but produce incentive salience and elicit goal-directed approach behavior only indirectly (as indicated by the dashed arrow from 'pleasure integrator' to the 'incentive salience attributor'). The direction of incentive salience attribution to stimuli that preceded or accompanied incentive salience activation is determined by associative learning. Thus, activation of the incentive salience attributor by a UCS results in incentive salience being assigned to the perception of conditioned stimuli that were originally neutral (such as the sight of a syringe) and to their mental representations. This is what makes conditioned stimuli attractive and 'wanted' and able to elicit approach. Conditioned stimuli (and UCS's) are always compared against past associative memories. Without the direction provided by associative learning, incentive salience could not be focussed upon any single target. Although diffuse attribution of incentive salience would be both psychologically and behaviorally activating, without associative direction it would not be sufficient to guide behavior towards a specific goal. Familiar conditioned stimuli that have been paired with incentive salience attribution in the past are the target of incentive salience when encountered again, especially when an animal is in particular physiological states (indicated by the arrow from 'physiological drive cues'). Incentive salience assigned to conditioned stimuli must be further 'reboosted' each time they are paired again with salience activation (indicated by the dashed arrow from the incentive salience attributor to associative learning). Disruption of this reboosting, by neuroleptics for example, can produce 'extinction mimicry' or decay of incentive value. Ordinarily, incentive salience is assigned only to stimuli that have been paired with pleasure. But brain manipulations (such as drugs or electrical brain stimulation) may circumvent pleasure, by activating the neural substrate of incentive salience directly. This will result in the attribution of incentive salience to associated stimuli and actions and result in their becoming 'wanted', even in the absence of pleasure. This can be considered a kind of 'sham reward' (see glossary entry for reward). Sensitization of the neural substrate for incentive salience will lead to pathological 'wanting' (craving) for stimuli associated with its excessive activation (e.g., those involved in drug taking), even if this produces little or no pleasure. As mentioned above, the direction of incentive salience by associative learning is the primary determinant of exactly which stimuli become craved. Thus, in the addict, drug-paired stimuli, which have been experienced repeatedly in association with the excessive stimulation of dopamine systems, become the nearly exclusive targets for the attribution of incentive salience. Other contributions of associative learning are also possible in this model. For example, the pleasure elicited by a UCS can change with repeated experience, as when one develops an appreciative palatc for Scotch whiskey (this is indicated in the model by the arrow from learning to the 'pleasure integrator'). Also, a CS that has been repeatedly paired with pleasure can come to itself elicit subjective pleasure, as in the example of a conditioned 'high' reported by 'needle freaks' (arrows from the CS to the 'pleasure integrator' via associative learning). But we suggest these effects are separate from the attribution of incentive salience and that they have only a relatively weak influence on motivated behavior, compared to the craving produced by the attribution of excessive incentive salience. Finally, we suggest that none of the psychological processes described in this model, except for subjective 'wanting' (craving) and subjective pleasure, are apparent to conscious awareness. The interaction among incentive salience, pleasure and associative learning is not available to introspection. Only the final products of the interaction are interpreted by cognitive mechanisms (not shown in the figure, see Nisbett and Wilson 228 ) as subjective 'wanting' and 'liking'. For an addict, whose neural substrates of incentive salience have been sensitized, the subjective product is dominated by the intense experience of drug craving. 
flails, etc.) that rats emit to tastes ${ }^{119}$. Much like the facial expressions that human infants display to sweet or bitter tastes ${ }^{319}$, these hedonic and aversive reactions reflect the perceived pleasure or displeasure of a taste sensation. The reactions of rats to taste are altered by many of the same things that control human perceptions of taste pleasure. The sensory pleasure of sweetness to humans, for example, is enhanced by hunger and suppressed by caloric satiety ${ }^{41,42}$. Hedonic reactions of rats to sweet tastes are similarly enhanced by hunger and suppressed by satiety ${ }^{26,43}$. The taste pleasure of a palatable food for humans can be abolished and replaced with subjective aversion by associative pairing of that food with gastrointestinal illness ${ }^{278}$. Similarly, hedonic reactions of rats to sweetness are abolished and replaced by aversive behavioral reactions after associative pairing of that taste with illness ${ }^{118}$. Finally, drugs that affect opioid or GABA neurotransmitters can enhance or suppress the hedonic reactions of rats to tastes in ways that should be expected based on current theories of the role of these neurotransmitter systems in taste pleasure ${ }^{75,230,340}$.

Application of the 'taste reactivity paradigm' to the role of mesotelencephalic dopamine systems in taste pleasure and motivated behavior leads to the conclusion that dopamine systems do not mediate taste pleasure, although they do mediate the incentive motivational properties of foods. There are three lines of evidence leading to this conclusion. (1) Dopamine antagonists do not decrease the sensory pleasure of tastes, measured by hedonic reactions, although they can decrease their incentive value. Conversely, dopamine agonists do not increase the sensory pleasure of tastes, although they can increase their incentive value ${ }^{340}$. (2) A bilateral neurotoxic lesion (6-OHDA), which depletes dopamine from the nucleus accumbens and caudate nucleus, does not diminish the hedonic evaluation of tastes, even though it completely abolishes the motivation to eat and renders natural incentives ineffective (ref. 28 and unpublished data). (3) Activation of the motivation to eat by electrical stimulation of the lateral hypothalamus, which is mediated in part by dopamine systems, does not potentiate the hedonic evaluation of tastes $^{27}$.

These experiments suggest, thereforc, that the role of dopamine systems in behavior motivated by food is not to enhance the sensory pleasure of tastes. Or, put another way, these experiments suggest that neural systems mediating 'wanting' food can be dissociated from neural systems mediating 'liking' food and that dopamine activates 'wanting'. This is what would be expected if dopamine mediates the salience of incentive stimuli, rather than the sensory pleasure evoked by incentive stimuli (Fig. 2). These findings are precisely what would be expected on the basis of our hypothesis that dopamine systems mediate the incentive motivational effects of drugs and are dissociable from other neural systems that mediate the subjective pleasurable effects of drugs and other stimuli. This kind of dissociation would explain the findings reported by Fischman and Foltin ${ }^{95}$ and by Lamb et al. ${ }^{192}$ (see above). You will recall that in these studies a low dose injection of cocaine or morphine motivated drug-taking behavior in 'postaddicts', but did not produce self-reported subjective pleasure.

- The second line of evidence that dopamine mediates incentive salience rather than sensory pleasure comes from a series of electrophysiological expcriments on the conditions under which dopamine neurons discharge in behaving animals ${ }^{288}$. When monkeys are first exposed to a novel situation dopamine neurons discharge to new, unexpected stimuli that produce orienting behavior. But these neuronal and behavioral responses soon habituate ${ }^{199}$. Dopamine neurons also respond when an animal encounters a natural incentive, such as when it touches food located out-of-sight or, in a learning task, when liquid in delivered to the mouth ${ }^{199,287}$. However, when a neutral stimulus (e.g., light) is paired with the availability of a natural incentive, dopamine neurons soon stop responding to the natural incentive and start to discharge most vigorously in response to the newly established conditioned incentive stimulus ${ }^{199}$ (also sec ref. 217). Dopamine neurons do not discharge when the animal actually eats the food, which they should if dopamine mediated the sensory pleasure associated with incentives (ref. 330 as well). Similarly, Kosobud et al. ${ }^{183}$ reported in a recent poster that in rats trained to bar press for sucrose, VTA unit activity increased prior to the presentation of sucrose. Dopamine neurons did not increase activity after sucrose presentation, when presumably the animal would experience sensory pleasure. Finally, the activity of dopamine neurons can be dissociated from non-incentive aspects of a situation and from the details of motor behavior, because their discharge is not coupled to "mnemonic or preparatory representational task components" (ref. 198, p. 337), to the execution of reaching movements to obtain and retrieve food or to a light unassociated with food ${ }^{199,287,289}$. Studies in cats also suggest that VTA dopamine neurons do not discharge in relation to most phasic movements ${ }^{330,341}$.

In summary, dopamine neurons discharge under conditions consistent with an attribution of incentive salience hypothesis of dopamine function ${ }^{27,28}$. They change their rate of discharge to a stimulus as the incentive value of the stimulus changes; as the stimulus 
becomes more or less salient. Indeed, Schultz ${ }^{288}$ concluded that their electrophysiological experiments "are consistent with the conclusion that dopamine neurons respond specifically to salient stimuli that have alerting, arousing and attention-grabbing properties" ( $p$. 134, our italics) *.

- A third line of evidence that dopamine mediates incentive salience rather than sensory pleasure comes from recent studies using high speed chronamperometry to measure nucleus accumbens dopamine activity during i.v. self-administration of heroin or cocaine ${ }^{115,172}$. In trained animals the first few drug injections greatly elevated a dopamine-related electrochcmical signal. However, detailed analysis of the time course of changes in the dopamine-related electrochemical signal, relative to subsequent injections, revealed that the dopamine-related signal increased prior to drug selfadministration, peaked at the time a response was inititated and then significantly decreased immediately following drug infusion. This is consistent with the view that dopamine mediates the incentive salience attributed to a drug-associated stimulus (presumably the lever in this case), because as extracellular dopamine increased drug 'wanting' would increase, to the point that an animal would initiate another drug infusion. The results are not consistent, however, with the view that dopamine mediates subjective pleasure (in which casc dopamine should risc after drug administration). Neither are they consistent with the view that drug responding is initiated by withdrawal symptoms associated with dopamine depletion, as suggested, for example, by Dackis and Gold ${ }^{64}$. A dopamine depletion hypothesis would predict that animals should initiate a drug infusion when the dopamine signal is at its lowest, not highest. Of course, a dopamine depletion/ withdrawal hypothesis, such as that proposed by Dackis and $\mathrm{Gold}^{64}$, also suffers from the shortcomings associated with all negative reinforcement hypotheses of addiction, which were discussed above.

- The fourth line of evidence that dopamine is more likely to produce enhanced incentive salience than enhanced drug pleasure comes from a consideration of the relative patterns of change in drug 'wanting' vs. drug 'liking' reported by human addicts during the gradual development of addictive behavior (Fig. 3). The pleasure induced by drugs does not increase if the dose of a drug is held constant over the course of repeated administrations. In fact, if pleasure changes

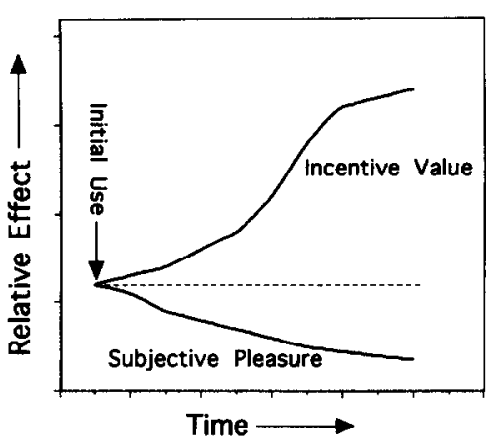

Fig. 3. A schematic illustration of the hypothetical relationship between changes in the incentive value of drugs and drug-related stimuli (drug 'wanting') vs. the subjective pleasurable effects of drugs (drug 'liking') during the development of an addiction. The development of an addiction is characterized by an increasing dissociation between the incentive properties of drugs, which gradually increase and the subjective pleasurable effects of drugs, which are shown here to slightly decrease (develop tolerance; but also see text and Note 5 in Ch. 6).

at all it decreases with repeated administrations (although, see Note 5 in $\mathrm{Ch}$. 6). If increased synaptic activity in sensitized dopamine systems were the neural substrate of pleasure, a given dose should produce more and more pleasure with repeated experience, rather than less and less (or no change).

Although the pleasure produced by a drug does not increase for human addicts, the craving for the same drug does increase with repeated experience (Fig. 3). An increase in 'wanting' drugs, as evidenced by self-report and by progressively more compulsive drug-seeking and drug-taking behavior is, of course, the defining characteristic of drug addiction. An increase in drug craving without an increase in drug pleasure cannot by explained on the assumption that sensitization enhances a dopaminergic mechanism mediating the subjective pleasure of drugs (see Note 3 in Ch. 6). But this increasing dissociation between drug 'wanting' and drug 'liking' is precisely what would be expected if enhanced activity at dopamine synapses were the neural substrate responsible for incentive salience (see Note 4 in Ch. 6).

\section{ELABORATION OF THE INCENTIVE-SENSITIZA- TION THEORY AND ITS IMPLICATIONS}

In this section we will elaborate more precisely how the major features of addiction are explained by the Incentive-Sensitization Theory of Addiction and discuss implications of the theory. To summarize the

\footnotetext{
\# Schultz ${ }^{288}$ tentatively suggested that the common psychological process underlying the discharge of dopamine neurons may be "motivational arousal", but also noted this was not completely satisfactory because "dopamine neurons respond every few seconds to the same stimuli over several tens of trials without habituation ${ }^{199,275,287,289}$, whereas arousal should be a longer lasting state not repeatedly induced at such short intervals" ( $p$. 135). We suggest that the conditions under which dopamine neurons discharge are better described by the hypothesis that
dopamine neurons attribute incentive salience ${ }^{27,28}$.
} 
central elements of the theory, we posit the following. (1) Many addictive drugs have in common the ability to enhance mesotelencephalic dopamine neurotransmission and to engage related structures (but see Note 4 in Ch. 6). (2) A psychological function of this neural system is to attribute incentive salience to the perception and mental representation of stimuli and actions, especially those that have been associated with activation of the system; to cause them to become highly salient, attractive and 'wanted'. (3) Repeated and intermittent administration of addictive drugs leads to incremental neuroadaptations in this neural system, which render it increasingly and perhaps permanently hyperresponsive (sensitized). (4) Associative control of this sensitized neural system causes tremendously enhanced incentive salience to be attributed to the act of drug taking and to stimuli associated with drug taking (i.e., to the acts and stimuli most closely associated with hyperactivation of dopamine systems); and thus, in the addict, drugs come to be pathologically 'wanted' (craved). (5) Sensitization of the neural system responsible for incentive salience can motivate addictive behavior (compulsive drug seeking and drug taking) independent of other motivating factors, such as the expectation of drug pleasure or the aversive properties of withdrawal. The associative targeting of sensitized incentive salience to drug-related stimuli results in the persistence of addictive behavior even in the face of many disincentives: for example, the loss of reputation, job, home and family (see Note 6 in Ch. 6).

We have argued that addictive behavior is motivated by the excessive 'wanting' of drugs (drug craving). Therefore, the first major issue we need to address is: why do addicts crave drugs independently of drug pleasure and withdrawal? What is the neuropsychological process that results in obsessive craving for drugs, leading to compulsive drug-seeking and drug-taking behavior, even when the drug may produce little pleasure? The second fundamental question we need to address concerns why drug craving persists for so long after the discontinuation of drug use and after the cessation of withdrawal symptoms; i.e., the nature of relapse. After this we will discuss the implications of Incentive-Sensitization for individual differences in the propensity to addiction, for therapy and the relationship between Incentive-Sensitization and other views of addiction.

\subsection{The independence of drug craving from drug plea- sure and withdrawal}

The Incentive-Sensitization Theory of Addiction provides an unique neuropsychological explanation for drug craving. Drug craving is the subjective experience that accompanies the attribution of excessive levels of incentive salience to drug-related stimuli (or their mental representations), due to sensitization of dopamine systems (see Note 4 in Ch. 6). Thus, drug craving is considered by this hypothesis to be a psychological process that is distinct from conditioned withdrawal signs and from either drug-induced pleasure or a conditioned 'high'. Other views of addiction often consider craving to be identical with or the direct result of, either conditioned withdrawal or a conditioned 'high'. For example, Childress et al. ${ }^{50}$ state: "We have often used the terms 'conditioned withdrawal' and 'conditioned craving' almost interchangeably, with the assumption that craving might be a form of mild withdrawal" (p. 38). But Childress et al..$^{50}$ go on to say: "Our patients did not, however, always subscribe to this position; reports of craving usually showed low correlations with reports of withdrawal. To paraphrase one indignant user, 'No, doc, craving is when you want it - want it so bad you can almost taste it... but you ain't sick... sick is, well, sick' ". To this addict withdrawal sickness clearly is separable from drug craving ${ }^{212}$. Neither is craving equivalent to a conditioned 'high', because reports of conditioned 'highs' are uncommon and are thus dissociable from the more frequent occurrence of both conditioned withdrawal signs and conditioned craving, as discussed earlier ${ }^{50,229}$.

The Incentive-Sensitization view of addiction is in agreement with the indignant user cited above; craving is pathological 'wanting'. It is not due to sickness. It is distinct from both the unpleasant symptoms of withdrawal and from drug pleasure. This view is supported by studies directly relating self-reported craving induced by exposure to drug-associated stimuli to ratings of withdrawal-like symptoms, drug-like effects and 'outcome expectancies' ${ }^{80,253}$. Although exposure to drug-related stimuli produced a significant increase in self-reported craving, as well as drug-opposite and drug-like effects "in a simple additive model the combined effects of positive outcome expectancies, cuespecific dysphoria and cue-specific drug-positive reactions were able to predict $28 \%$ of the variance in cue-specific craving."... "A much larger proportion of the variance in craving remains unexplained by these factors" (ref. 253, p. 1142-1143). We suggest the reason there is only a weak relationship between these variables and drug craving is that they do not cause craving. Craving is due to excessive activity in a separate and sensitized neuronal system that mediates the attribution of salience to incentives. This is a neuronal system that normally mediates the 'wanting' of things in the environment. Although this neuronal system usually functions in concert with neuronal systems that 
mediate pleasure ('liking'), in the addict the normal link between these systems is disrupted and pathological levels of 'wanting' become dissociated from 'liking'. We think this dissociation accounts for the unusual psychological profile of an addict: intense drug craving separated from the normal pleasures and punishments of life *.

In this light the irrationality of addictive behavior, which is discussed so eloquently by Falk et al..$^{86}$, starts to make some 'sense'. The irrationality of the behavior is due to an increasing dissociation between the incentive properties of drugs (incentive salience) and their subjective pleasurable effects. Becausc the process of salience attribution can be activated, independent of subjective pleasure, incentive salience can be strong even if pleasure is weak or absent. This is one reason why there is not always a strong correlation between the incentive motivational properties of drugs and their hedonic properties. It is also why people will self-administer low doses of drugs that do not produce subjective pleasure ${ }^{95,192}$. Furthermore, the attribution of incentive salience is not a conscious process and the introspective experience of 'wanting' or craving is only a person's interpretation of the outcome of that process. Much of the time the attribution of incentive salience may be more implicit than explicit ${ }^{228}$. Regardless, the addict can be only subjectively aware of the outcome of excessive incentive salience attribution, craving. The addict may have little insight into the reason for the craving and indeed, may himself be bewildered by its intensity. At a conscious level addicts may recount all of the negative consequences of continued drug use, deplore their situation, even comment that the drug does not continue to give great pleasure - and not understand why their craving persists.

\subsection{The development of addictive behavior}

The Incentive-Sensitization Theory can explain why the development of an addiction is typically a gradual, progressive process. The attribution of a high level of incentive salience to drug-associated stimuli and the pleasurable effects of drugs, increase the probability drug-related stimuli will attract attention and that drugs will be sought out in the future. If drug use continues, dopamine systems become progressively more sensitized. With each repetition greater and greater incentive salience is attributed to drug-related stimuli and the associative pairing of drug-related stimuli with the intense activation of dopamine systems produced by drugs leads to an increasing focus of salience attribution upon just these stimuli. Thus, 'wanting' is gradually transformed into craving, drugs become craved to the relative exclusion of all else, and drug-associated stimuli elicit this craving independent of any pleasure they produce. In short, the developing addict comes to 'want' drugs more and more because drug-related stimuli become imbued with greater and greater incentive salience, even though at the same time drugs may be 'liked' less and less.

\subsection{Relapse: drug-induced drug craving}

Drug craving sometimes remains high or is even increased immediately after drug administration, when the drug is producing subjective pleasure, as has been reported for alcohol, cocaine, heroin and hydromorphone ${ }^{80,96,150,215,325}$. This is the proverbial drink that whets the appetite and leads to relapse. Why should this be? As pointed out earlier, this is not consistent with a negative reinforcement view of craving ${ }^{329}$, because the drug should eliminate withdrawal symptoms ${ }^{* *}$. Neither is it consistent with a pleasure-seek-

\footnotetext{
* Other brain manipulations also are known to 'fracture' behavioral or psychological subsystems whose operations are so intertwined that one's subjective experience is of only one process. An example is the phenomenon of 'blindsight ${ }^{350}$, which refers to the ability of people rendered blind by an occipital cortex lesion to accurately localize visual stimuli presented in their blind visual field, despite having no conscious awareness of perceiving any stimulus. People have no subjective experience of separate psychological processes (and neural systems) underlying the identification vs. the localization of visual stimuli. But an occipital lesion 'fractures' these processes, revealing two distinct psychological processes where there appeared to be only one. Another example is the dissociation of declarative (explicit) and procedural (implicit) memory systems seen following damage to the medial temporal lobes ${ }^{318}$. We are not subjectively aware that distinct neural systems are involved in learning to solve puzzles (for example, the Tower of Hanoi puzzle) and learning facts (for example, learning a list of words). But following a bilateral medial temporal lobe lesion the ability to learn and remember facts is lost, whereas the ability to learn and remember puzzles is left intact; although the latter occurs in the absence of conscious awareness. We think that the dissociation between 'wanting' and 'liking' seen in addicts represents a 'fracture' of psychological processes akin to 'blindsight' and the implicit/explicit memory distinction. That is, repeated drug use changes the brain, as a lesion changes the brain, revealing two distinct psychological processes where there subjectively appeared to be only one. Furthermore, like the localization of visual stimuli and procedural learning, the attribution of incentive salience is 'implicit'; it often may occur in the absence of conscious awareness.

** Except according to Solomon's opponent-process theory ${ }^{312}$, which is the only negative reinforcement theory that can successfully explain relapse induced by re-exposure to the drug. According to Solomon's theory, re-exposure to the drug elicits a moderate a-process or drug-like effect, which in turn triggers the still strong b-process or drug-opponent effect. The problems faced by the opponent-process theory, however, are: (1) it relies entirely upon withdrawal symptoms to motivate addictive behavior and is thus liable to the general criticisms that we described for negative reinforcement theories of addiction; (2) it posits the growth of an opponent-like process during addiction, for which there is no direct evidence other than the phenomena of tolerance and withdrawal themselves (and which is contradicted by evidence discussed above that the incentive properties of drugs show sensitization rather than tolerance); and (3) it posits the opponent-process to be elicited only and always by the a-process, whereas evidence exists that withdrawal and drug pleasure have separate, independent neural
substrates ${ }^{365}$.
} 
ing view, because a dose sufficient to produce subjective pleasure should satisfy or at least reduce the craving; not exacerbate it. Drug craving at the time of drug taking is consistent, however, with an IncentiveSensitization view. According to Incentive-Sensitization craving is the subjective experience associated with incentive salience attribution. Because many addictive drugs increase dopamine activity, which produces incentive salience, one would expect drug administration to produce drug 'wanting'.

Indeed, there is considerable evidence that re-exposure to drugs can reinstate compulsive drug-seeking and drug-taking behavior ${ }^{83,325,326}$. As pointed out by Stewart et al. ${ }^{326}$ : "The idea that ingestion of a formerly abused drug induces a strong motivational state or craving for the drug and that it retains the ability to reinstate this craving over an indefinite period of abstinence from the drug is not new. One of the basic tenets of Alcoholics Anonymous (anonymous, 1939) is that people who have at one time shown uncontrolled drinking and physical dependence are permanently unable to drink moderately; one drink is said to elicit an urge to have another" (p. 257). This phenomenon is usually explaincd by 'priming' and Stewart et al. ${ }^{326}$ have argued that 'priming' reinstates drug use because "the presence of the drug in the body (not its absence) activates appetitive motivational mechanisms that are involved in the reinitiation of drug seeking behavior" (p. 253). An involvement of dopamine in priming is suggested by reports that the infusion of morphine directly into the ventral tegmental area is sufficient to prime responding for i.v. heroin or cocaine, intra-accumbens amphetamine can prime responding for i.v. heroin ${ }^{325,326}$, and haloperidol prevents priming for amphetamine ${ }^{83}$. It is also relevant to note that craving in long-term abstinent cocaine abusers (humans) has been associated with elevatcd plasma and CSF levels of the dopamine metabolite, $\mathrm{HVA}^{173,206}$.

Stewart's view is entirely consistent with the Incentive-Sensitization Theory of Addiction presented here. We would add only two additional points. First, an Incentive-Sensitization view of addiction identifies the "appetitive motivational mechanism" mentioned by Stewart et al. ${ }^{326}$ specifically as incentive salience and not, for example, drug pleasure. Second, we hypothesize that the ability of drugs to produce incentive salience is progressively increased (sensitized) by repeated exposure to drugs because drugs sensitize mesotelencephalic dopamine systems. Thus, in highly sensitized individuals, such as addicts, relapse is the rule rather than the exception, especially after a priming 'taste', because this acts on a hypersensitive neural system that mediates incentive salience - eliciting pathologically strong 'wanting' (craving) and thus relapse.

\subsection{Relapse: interactions between different drugs and the effects of drug-related stimuli}

Not only can the preferred drug of abuse reinstate addictive behavior, but often other drugs can as well; and addicts usually use more than one drug (which is significant in itself). In animals too, priming can occur across drug classes. For example, i.v. amphetamine or bromocriptinc can prime the self-administration of heroin ${ }^{367}$ and i.v. morphine can prime responding in animals trained to self-administer cocaine ${ }^{326}$. Furthermore, dopamine systems have been implicated in priming between drug classes. An intra-accumbens injection of amphetamine, which selectively activates the mesolimbic dopamine system, can prime responding for i.v. heroin ${ }^{327}$ and intra-ventral tegmental area morphine can prime responding for i.v, cocaine ${ }^{322}$.

According to the Incentive-Sensitization view of addiction, drugs can prime responding for each other because the same dopamine systems are activated by each and dopamine mediates the incentive salience attributed to many different drugs. Therefore, if dopamine systems become sensitized by past drug use it would be expected that a second, novel drug would be able to prime responding and precipitate relapse, as long as the second drug also activates hypersensitive dopamine systems. This idea was proposed previously by Stewart and Vezina ${ }^{327}$, who argued that the ability of opiates and stimulants to prime responding for one another "may be related to the ability of opiates and stimulant drugs to cause sensitization" (p. 287), within dopamine systems. In support of this view, cross-sensitization has been reported to the psychomotor stimulant effects and to the incentive motivational effects of a number of drugs, as well as in the ability of drugs to elevate dopamine neurotransmission (see above for references).

Not only may re-exposure to drugs themselves precipitate relapse, but environmental stimuli associated with drugs are known to induce craving and precipitate relapse in humans ${ }^{50,229}$ and prime drug responding in animals $^{325,326}$. This evidence has been reviewed by Stewart et al. ${ }^{326}$ and need not be reiterated here. It is consistent, however, with an Incentive-Sensitization view of addiction. In this view sensitization of a neural substrate responsible for incentive salience becomes expressed as addictive behavior largely by enhancing the incentive properties of stimuli associated with drugs. Heightened incentive salience is focussed on these stimuli and their mental representations by associative learning processes and they become the elicitors 
and objects of craving. Even in the absence of the drug and long after withdrawal signs have faded, drug-related stimuli remain potent conditioned incentives able to elicit the attribution of incentive salience. Indeed, in the context of an Incentive-Sensitization view of addiction this is why environmental stimuli associated with drugs are extremely effective in precipitating relapse in addicts (however, see Note 8).

This view is an alternative to both the conditioned 'high' interpretation and the 'explicit memory' interpretation of relapse discussed above in the section on positive reinforcement/euphoria theories. We suggest the effects of drug-related stimuli on relapse are independent of both drug cue elicited feelings of pleasure (a conditioned 'high') and of the explicit memories of drug taking such cues might elicit. You will recall we argued that an 'explicit memory' hypothesis of relapse places an extraordinary explanatory burden on the assumption that relapse-provoking memories are qualitatively different from the myriad other memories of drugs that do not provoke relapse. We suggest, to the contrary, that conscious remembering in response to cues may be essentially similar to explicit memories that have gone before, neither more vivid nor qualitatively different. The difference in the processes triggered by an effective drug-paired context, which results in relapse when earlier memories did not, may be in associative incentive systems that are not explicitly available to consciousness.

Incentive salience is such an associatively triggered process. It occurs in the absence of awareness and its operation requires no qualitative difference in explicitly conscious memory in order to provoke relapse. Unlike explicit memories, the attribution of incentive salience is an implicit process. It is governed by the laws of associative learning and is influenced by factors that control other forms of implicit learning. Chief among these controlling influences is the gating role of associative context ${ }^{259}$. Context refers to the entire configuration of situational stimuli in which the CS has been learned. Associative context can modulate the effectiveness of any CS. Why then should relapse occur at a particular moment, rather than during earlier memories or earlier encounters with drug-paired stimuli? Presumably because of variations in the completeness of the associative context. The greater the extent to which contextual factors, such as mood, environment and other situational variables, mimic the context of previous drug taking, the more likely relapse will occur.

\subsection{Relapse: the role of stress}

Relapse to compulsive drug use is not always precipitated by re-exposure to a drug or even by specific environmental stimuli associated with drugs, but sometimes by ill-defined environmental circumstances; including mood changes evoked by stress ${ }^{49,229}$. A traditional view of why stress may lead to relapse is that it prompts 'escape' from an unpleasant situation via drug taking. An alternative possibility is that sensitization of incentive salience could play a role in stress-induced relapse because addictive drugs and stress both activate dopamine systems and both sensitize dopamine systems ${ }^{8,9,160,268}$. As discussed above, animals previously exposed to drugs such as amphetamine, cocaine or morphine are later hyperresponsive to stress and animals exposed to repeated intermittent stress are later hyperresponsive to the psychomotor stimulant and incentive motivational properties of drugs ${ }^{243}$. According to an Incentive-Sensitization view stress may induce craving and relapse because, by activating dopamine systems, stress would magnify the incentive salience attributed to environmental stimuli. Environmental stimuli that were especially potent as incentives, such as drug-associated stimuli for addicts, would be the focus of enhanced salience due to their associative history. Drug-associated events would become especially craved again as a consequence of stress (see Note 9 in Ch. 6).

It is interesting to speculate that the converse sequence of events could also occur. That is, prior exposure to repeated intermittent stress may predispose susceptible individuals to drug addiction by sensitizing those neural systems that mediate the incentive motivational effects of drugs ${ }^{69,159,268}$. In such individuals the incentive motivational effects of an initial drug experience may be significantly enhanced because of drug action on a previously sensitized neural substrate. This would increase the probability that these individuals would show subsequent drug-seeking and drug-taking behavior. Indeed, experimental evidence for such a phenomenon has been reported by Piazza et al ${ }^{243}$, who found that past experience with stress (repeated tail pinch) facilitated the subsequent acquisition of amphetamine self-administration behavior in rats.

\subsection{Individual differences in the propensity to addiction}

The last feature of addiction we will discuss concerns the fact that the majority of people in this country at some point experiment with drugs, but most do not become addicts. For example, over $55 \%$ of 18-34 yr olds have at one time sampled illicit drugs (e.g., marijuana, inhalents, cocaine, heroin or hallucinogens; NIDA National Household Survey on Drug Abuse, 1991). Why do the vast majority of these people not develop an addiction? Why are some individuals more susceptible to addiction than others? Social fac- 
tors are important, of course, but even persons from very similar backgrounds differ greatly in their tendency to develop addictive behavior. If, as proposed here, drug-induced neuroadaptations underlying sensitization play a central role in the development of addiction: (1) there should be large individual differences in the susceptibility to sensitization and (2) individual differences in the susceptibility to sensitization should be related to the propensity to addiction.

There are indeed enormous individual differences in the susceptibility to sensitization, a point that has been emphasized by a number of researchers ${ }^{190,194,268,294}$. Some of this individual variation is due to genetic variation, because among animals there are marked strain differences in the susceptibility to sensitization. Strain differences in both rats and mice have been reported in the sensitization produced by repeated treatment with amphetamine $e^{110,194,268,300}$, cocaine ${ }^{304}$, ethanol $^{60,142,240}$ and morphine ${ }^{302}$. There are also marked strain differences in mesotelencephalic dopamine systems ${ }^{14,91,145}$, but we know of no studies directly relating strain differences in the susceptibility to sensitization to strain differences in mesotelencephalic dopamine systems. Nevertheless, it is important that initial studies with recombinent-inbred lines of mice suggest that the genetic determinants of acute responsiveness to drugs are dissociable from those responsible for susceptibility to sensitization ${ }^{304}$. Many behavioral genetic studies on drug responsiveness have focussed on variation in the acute response to drugs, not susceptibility to sensitization. But the Incentive-Sensitization Theory suggests that the susceptibility to sensitization may be most relevant for the development of addictive behavior and therefore, information on genetic factors leading to high susceptibility to sensitization may be of particular importance in understanding the genetics of addiction.

A number of other factors have been reported to influence individual differences in the susceptibility to sensitization including, age ${ }^{100,175,303}, \operatorname{sex}^{45,46,107,250,267,273}$ and hemispheric differences in dopamine systems ${ }^{190}$. Whether the influence of these variables on the susceptibility to sensitization is causally related to the propensity to self-administer drugs or to related variation in dopamine systems is not yet known, although correlative relations have been reported ${ }^{47,108,109,294}$.

There are, however, a number of interesting studies on behavioral traits that do predict both the susceptibility to sensitization and the propensity to self-administer amphetamine ${ }^{241}$. For example, responsivity to novelty is reported to predict susceptibility to sensitization $^{133-135.242}$. Similarly, animals that eat and drink in response to electrical stimulation of the lateral hy- pothalamus (not all do) show an enhanced susceptibility to amphetamine sensitization ${ }^{220,221}$. Most importantly, these same traits are correlated with a propensity to acquire amphetamine self-administration ${ }^{241}$ and with differences in dopamine dynamics in the nucleus accumbens ${ }^{36,132}$. Individual differences in reactivity to novelty, in amphetamine sensitization, and in amphetamine self-administration may involve variation in the responsiveness of the hypothalamo-pituitary-adrenal (HPA) axis. Animals that show a high response to novclty also show a prolonged elevation in plasma corticosterone in this situation, relative to low responders ${ }^{190,241}$. That the HPA axis may play a role in drug sensitization is suggested by experiments showing that activation of the HPA axis is necessary to induce sensitization to amphetamine or stress ${ }^{54,55,69}$, perhaps by the action of corticosterone on glucocorticoid receptors $^{261}$ (cf. ref. 57) and by a report that repeated exposure to exogenous corticosterone sensitizes animals to a subsequent amphetamine challenge ${ }^{244}$.

\subsection{Implications of the Incentive-Sensitization Theory for Therapy}

There is considerable interest in developing effective therapies for the treatment of drug addiction, but this has proven to be a very difficult problem. The Incentive-Sensitization view of addiction may provide some insight as to why effective therapies have been elusive, and potentially, may point the way to the development of more effective approaches.

5.7.1. Extinction training. A recent trend in the psychotherapeutic treatment of addiction is based on the recognition that drug-conditioned stimuli are very potent in eliciting craving and precipitating relapse ${ }^{229}$. There have been attempts, therefore, to 'extinguish' conditioned responses to such stimuli. Indeed, the repeated presentation of drug-related stimuli, in a laboratory setting, results in a progressive decline in drug craving elicited by druy-related stimuli ${ }^{50,229}$. It is interesting, however, that some of the autonomic responses to such stimuli are more resistant to extinction than the subjective effects, and non-specific changes in mood state (especially anger) can rapidly reinstate conditioned stimulus-induced drug craving ${ }^{49}$.

In the context of the Incentive-Sensitization Theory this might occur because the neuroadaptations underlying sensitization persist, despite extinction of the conditioned stimulus control of sensitization. That is, the ability of conditioned stimuli to control the expression of sensitization may be thought of as learning-related neuroadaptations layered 'on-top' of the neuroadaptations responsible for sensitization, but which do not directly alter or reverse the neuronal changes responsi- 
ble for sensitization. Also, contextual factors that control the associative attribution of incentive salience may not transfer between the clinic and the street. Thus, extinction training may extinguish responses to specific stimuli under specific circumstances, but other non-target stimuli can still access sensitized neural systems mediating incentive salience, as can environmental stress. On the positive side, the fact that the expression of sensitization can be brought under strong conditioned stimulus control ${ }^{323}$ suggests it should be possible to develop learning strategies to control the output of sensitized neural systems. However, the persistence of the neuroadaptations underlying sensitization and their resistance to extinction ${ }^{328}$, suggests that coping with addiction may be a very long, ongoing process. Of course, this has been recognized for many years by organizations like Alcoholics Anonymous. For example, Anonymous states: "We know that while the alcoholic keeps away from drink, as he may do for months or years, he reacts much like other men. We are equally positive that once he takes any alcohol whatever into his system, something happens, both in bodily and mental sense, which makes it virtually impossible for him to stop" (ref. 7, p. 22).

5.7.2. Pharmacotherapeutic approaches. The IncentiveSensitization Theory of Addiction also has implications for the development of effective pharmacotherapies. The two major pharmacotherapeutic approaches at present either target the treatment of withdrawal symptoms or involve drug substitution therapy (e.g., methadone maintenance). The Incentive-Sensitization Theory predicts neither of these approaches will be very successful in eliminating addictive behavior, because neither target the fundamental neuroadaptations underlying sensitization. Of course, many years of experience with opiate addicts have already shown that the alleviation of withdrawal is not an effective longterm solution for addiction ${ }^{363}$ and the drugs used in substitution 'therapies' usually are addictive themselves ${ }^{363}$. Furthermore, some drugs used to treat withdrawal also may induce sensitization ${ }^{89,202,203,277}$.

An Incentive-Sensitization view of addiction suggests that to really 'cure' addiction agents need to be developed that directly target and reverse the neuroadaptations underlying sensitization. There are presently a number of agents known to prevent the development of sensitization, including dopamine antagonists (see above for references) and glutamate antagonists ${ }^{161,164,369}$ (cf. ref. 342). Unfortunately, these compounds do not reverse the neuroadaptations underlying sensitization, but only prevent its development if they are given every time the addictive drug is given. This is not a practical approach for the treatment of addiction (see Note 1 in Ch. 6). The Incentive-Sensitization Theory of Addiction predicts that an especially effective pharmacotherapeutic agent would reverse sensitization-related neuroadaptations. However, to our knowledge, no one has identified such a compound. Of course, any rational drug design program will require that we know a lot more about the nature of sensitization-related neuroadaptations than we know at present.

\subsection{Relationship between incentive sensitization and other views of addiction}

In closing, we want to emphasize that the Incentive-Sensitization Theory of Addiction does not exclude other factors that contribute to drug-taking behavior. For example, the Incentive-Sensitization Theory does not address a number of features of drug use, including why people experiment with drugs in the first place (experimental drug use), casual (not addictive) patterns of drug use or why people often use drugs that do not usually lead to compulsive patterns of use (e.g., LSD; see also Note 7 in Ch. 6). The Incentive-Sensitization Theory of Addiction does not preclude a role for pleasure-seeking and withdrawal avoidance in drug-taking behavior. These different views of addiction are not mutually exclusive, unless they are taken as the sole explanations for addiction. There can be no doubt that addiction results from very complex interactions amongst social, cultural, economic, psychological and biological variables. These complex interactions determine whether experimentation with drugs first occurs, whether further drug use is sustained and whether drug use leads to addiction. Exactly which factors motivate behavior will vary over time and across different drugs. The Incentive-Sensitization view does not exclude the possibility, for example, that an explicit memory of drug pleasure or a conditioned 'high' could contribute to a desire to repeat the drug experience in some situations. This may be especially true early in the development of an addiction $^{363}$. Early in the development of an addiction, before marked sensitization has occurred, the memory of the subjective pleasurable effects of drugs could be a major factor motivating drug-taking behavior. For example, it has been suggested that the initial subjective effects of drugs can predict later drug habits ${ }^{120}$. Also drug-taking behavior is influenced initially to a great extent by social factors, such as peer pressure.

Neither does the Incentive-Sensitization view of addiction deny that the unpleasant symptoms of withdrawal could motivate drug taking in some individuals, under some circumstances, in order to relieve symptoms. The role of withdrawal avoidance may vary greatly 
from drug-to-drug. In some respects drug taking motivated by pleasure-seeking or withdrawal avoidance seems 'normal'; that is, the reasons for drug taking are understandable to a non-addict. For example, recent suggestions that drug craving is caused by short-term dopamine depletion after drug use are based largely on the assumption that craving is merely a rational response to withdrawal symptoms ${ }^{63,204,351}$. But by our view this is not craving; nor is it the fundamental problem in addiction. Craving is obsessive, irrational, pathologically intense drug 'wanting' for no obvious reason, which leads to compulsive drug-seeking and drug-taking behavior. Craving is difficult for both the addict and the non-addict to understand and this is what we propose is primarily due to sensitization of incentive salience.

The Incentive-Sensitization Theory of Addiction is also compatible with the 'Psychomotor Stimulant Theory' of Wise and Bozarth ${ }^{365}$. Wise and Bozarth ${ }^{365}$ argued that addictive drugs have in common the ability to induce 'psychomotor activation', which was proposed to be due to activation of a common biological mechanism associated with approach behavior and mediated by dopamine. This biological mechanism is thought to be fundamental in producing reinforcement ${ }^{111,343}$. The fact that the psychomotor-activating effects of addictive drugs are sensitized by repeated drug administration is certainly consistent with the 'sensitization' component of the Incentive-Sensitization Theory. But we further specify here that the psychological process responsible for 'reward-related' psychomotor activation is the attribution of incentive salience. Although incentive salience may lead to locomotion and approach, because this psychological process makes stimuli in the environment more salient, attractive and 'wanted', these functions may be separable ${ }^{260}$. For example, a brain manipulation could inducc locomotion, perhaps by activating brainstem locomotor pattern generators, without producing incentive salience; and incentive salience could be attributed in the absence of locomotion (for example, in a rat rendered cataleptic by morphine, but who still acquires a conditioned place preference). Thus, in our view it is specifically the sensitization of incentive salience that makes drugs and drug-associated stimuli increasingly attractive and 'wanted'. Increased psychomotor activation is just a correlate of sensitized incentive salience.

In closing, the ability of the Incentive-Sensitization Theory of Addiction to capture the 'essence' of addictive behavior (compulsive drug seeking and drug taking) can be illustrated by a 'thought experiment'. For the sake of argument, imagine that our assumptions regarding the criteria for an adequate theory of addic- tion are correct and that the neural system involved in assigning incentive salience to drugs and drug-associated stimuli is indeed dissociable from those mediating the subjective pleasurable effects of drugs. Now imagine that repeated intermittent drug use causes gradual and incremental changes in the neural system responsible for incentive salience, such that this neural system becomes very hypersensitive (sensitized). Further imagine that the expression of this sensitized system is focussed expressly on stimuli that have become associatcd with its excessive activation, so drugs and drug-associated stimuli become irresistibly attractive ('wanted') and thus able to control behavior. But the neural system(s) responsible for the subjective pleasurable effects of drugs either does not change or else becomes hyposensitive (tolerant). Finally, imagine that incentive salience is attributed in the absence of conscious awareness. Now consider what a creature with this brain would be like. An addict, we think.

\section{NOTES}

\subsection{Note 1. Role of control and intermittency of drug administration in sensitization}

6.1.1. Control. Although there have been many reports of sensitization to addictive drugs, it is important to acknowledge that in nearly all of these studies sensitization was induced by non-contingent drug treatment. That is, the animal's behavior had no influence on whether it received a drug or not. It is known, however, that drugs can produce different effects depending on whether they are given in a response-contingent or response-non-contingent manner ${ }^{309,310}$. Also, the behavioral sensitization to amphetamine or cocaine induced by footshock stress is influenced by whether an animal has control in the situation. For example, MacLennan and Maier ${ }^{200}$ reported that behavioral sensitization did not occur in rats who could control the duration of footshock, but did occur in rats receiving an identical amount of shock, but who had no control. It will be critical to determine, therefore, whether the response-contingent administration of drugs induces sensitization.

There have been very few studies directly addressing this issue. We are aware of one report of sensitization following experience with cocaine self-administration. Falk et al ${ }^{88}$ tested for behavioral sensitization by challenging animals with cocaine 7-10 days after the discontinuation of an oral cocaine self-administration regimen (involving a schedule-induced polydipsia paradigm). Cocaine-experienced animals showed a marked shift to the left in the dose-response curve for cocaineinduced locomotor activity. These data show that sensi- 
tization to cocaine can occur following responsecontingent drug administration, as well as following non-contingent drug administration. It may also be relevant that in self-administration studies animals are often given non-contingent drug injections prior to training or during shaping, because this facilitates the acquisition of a self-administration habit. It is possible the development of a drug self-administration habit is facilitated by these procedures because they produce the kinds of sensitization-related neuroadaptations under discussion here (although one also has to consider the possibility that prior non-contingent administration produces tolerance to the aversive properties of drugs). 6.1.2. Intermittency. On the other hand, sensitization was not found in a microdialysis experiment involving cocaine self-administration ${ }^{143}$. These researchers found that the ability of self-administered cocaine to elevate extracellular dopamine was actually decreased in drug experienced rats. However, in this experiment the dialysis test was given $24 \mathrm{~h}$ after the last self-administration session. Sensitization-related changes in dopamine neurotransmission often are not evident after such short periods of withdrawal, even following non-contingent drug administration ${ }^{158,176,295,296,370}$. This is probably because intermittency is a critical variable both in inducing sensitization and in its later expres$\operatorname{sion}^{232,248,268,272}$. If injections are given too close together in time tolerance, rather than sensitization, usually occurs. The development of sensitization is maximized by spacing injections far apart in time (2-3 days to a week). Similarly, if a challenge injection is given within the first few days after the discontinuation of escalating dose amphetamine treatment behavioral sensitization is not evident. But if a challenge injection is given after a longer period of withdrawal, from 1 week to 1 year, animals are markedly sensitized ${ }^{232}$. Thus, sensitization may not be apparent after self-administration regimens that allow animals to maintain elevated brain levels of a drug for prolonged periods of time, especially if animals are tested soon after the end of a bout of self-administration.

It is intriguing that intermittency is not only critical for inducing sensitization, but is thought to play an important role in the development of many persistent and habitual behaviors, including addictive behavior ${ }^{86}$. Of course, drug-taking behavior in human addicts is often characterized by intermittency. Drugs are frequently taken in 'runs' of self-administration, interspersed with 'crashes' lasting a few days. Intermittency may also be imposed because a considerable amount of time is required to obtain the money necessary to buy drugs. Falk and his colleagues ${ }^{86,87}$ have argued that such intermittent schedules of drug administration may greatly enhance the reinforcing properties of drugs and catalyze "drug overindulgence in humans". They state "when life's crucial commodities are in short supply and available only on intermittent, marginal schedules... drugs can become all-powerful in reinforcing efficacy" (ref. 87 , p. 1506). We would only add that the neural basis of this effect, in the context of Incentive-Sensitization, may involve a facilitation of sensitization-related neuroadaptations. We would expect that an intermittent pattern of drug self-administration, such as a cycle of 'runs' and 'crashes', would produce sensitization of dopamine neurotransmission and incentive salience $^{270}$.

\subsection{Note 2. Specific motivational effects of dopamine blockade}

The literature on the effects of dopamine antagonists on motivated behavior has been reviewed extensively and the reader is referred to papers cited in the text for comprehensive lists of citations. In brief, some of the most clearly motivational effects of dopamine antagonists include the following. (a) Mimicry of extinction by dopamine antagonists, in which instrumental responses for a food, drug or electrical brain stimulation decline only gradually after neuroleptic administration as though the reinforcer were no longer efficacious (the decline in response is not right away and does not occur unless the animal is allowed to perform the task $)^{99}$. (b) The associative reinstatement of instrumental performance after a dopamine antagonist has suppressed instrumental responding, by transferring the animal to a separate task in which it has previously been reinforced, but in which it has not yet experienced the drug, and in which it responds again at a high level even though the drug is still in effect (i.e., it must learn to 'extinguish' again in the second task) ${ }^{103}$. (c) Transfer between real extinction and extinction mimicry in 'resistance to extinction' paradigms, as though a dopamine antagonist drug were perceived by the animal as being similar to 'no reinforcer'. (d) Reward ${ }^{\dagger}$-specific 'curve shift' reductions in psychophysical paradigms that can distinguish between reductions in instrumental performance due to motor impairment versus reductions due to decreased reinforcement (refs. 78, 104 for example). (e) 'Reduced palatability' patterns of decrease in food consumption that mimic those produced by manipulations of the sensory pleasure of the food: for example, low doses of dopamine antagonists mimic the effects upon sucrose drinking that are produced by dilution of the sucrose solution $^{13,53,67,106,308,374}$. 


\subsection{Note 3. The neural substrate of drug 'liking' (plea- sure)}

We have argued that mesotelencephalic dopamine projections provide the neural substrate for drug 'wanting' (via attribution of incentive salience), but not for drug 'liking' (for the subjective pleasurable effects of drugs). This naturally raises the question: what is the neural substrate for drug 'liking'?; for the pleasurable affective states produced by addictive drugs? We cannot provide a definitive answer to this question, except to rule out dopamine, but we can point to other candidate neural systems. Chief among these are endogenous opioid neurotransmitter systems. Opioid agonists that increase motivated behavior towards food, such as morphine, do enhance the sensory pleasure of food as measured by the taste reactivity paradigm ${ }^{75}$. Similarly, activation of benzodiazepine-GABA systems within the brainstem enhances sensory pleasure by the taste reactivity measure, although it is not yet clear whether this depends on an interaction with brain opioid systems (ref. 340 for example). In summary, we do not know the neural substrate of drug pleasure, but these are a couple of candidate systems and future research may reveal others.

\subsection{Note 4. Dopamine, sensitization and incentive salience}

There are two issues regarding the neural system(s) responsible for sensitization of incentive salience that require further discussion. The first concerns elaboration of exactly which of the many different mesotelencephalic dopamine projection systems is likely to mediate incentive salience. We are well aware that there are multiple anatomically and functionally distinct mesotelencephalic dopamine projection systems. The extent to which the effects of manipulations of dopamine systems on incentive motivation are due to an action on any single one of these dopamine systems is not always clear. The contribution, for example, of dopamine projections to the frontal cortex, septum, caudate, accumbens (core vs. shell), olfactory tubercle or amygdala and the extent to which there may be interactions between these systems in the assignment of incentive salience, is, for the most part, unknown. We hesitate, therefore, to prematurely assign all responsibility for incentive salience or the sensitization of incentive salience, to a specific dopamine projection system. This is why we often use the broader term, mesotelencephalic dopamine systems. Nevertheless, we recognize that most of the evidence linking mesotelencephalic dopamine systems to incentive motivation primarily implicates the so-called mesolimbic dopamine projections to the ventral striatum (nucleus accumbens). Therefore, to the extent that the sensitization of incentive salience is mediated by a specific dopamine system it is probably the dopamine projection system to the ventral striatum. But we do not discount the possibility that other dopamine systems may play a role.

The second issue is whether only dopamine systems are involved in the sensitization of incentive salience. We have focused on dopamine systems as the site of sensitization-related neuroadaptations for the reasons described in the text. We acknowledge, however, that the neurobiology of drug craving involves much more than just a simple sensitization-related increase in dopamine neurotransmission and the neural substrate of incentive salience is surely much more complicated than this.

For example, Wise and Rompre ${ }^{368}$ have cautioned that "while the evidence is strong that dopamine plays some fundamental and special role in the rewarding effects of brain stimulation, psychomotor stimulants, opiates and food, the exact nature of that role is not clear. One thing is clear: dopamine is not the only reward transmitter and dopaminergic neurons are not the final common path for all rewards" (p. 220). We agree with Wise's caution. To influence motivated behavior via the attribution of incentive salience, dopamine systems must interact with many other neural systems, especially those involved in hedonics and associative learning. It is also possible that some incentives may not directly activate dopamine systems at all $^{181}$ and it is conceivable that some behavior may be controlled by non-incentive based processes, such as Skinnerian S-R reinforcement, Hullian drive-reduction or goal-directed computational procedures (e.g., TestOperate-Test-Exit procedures ${ }^{216}$ ) (see Toates ${ }^{339}$ for a discussion of how to distinguish some of these alternatives).

The phenomenon of behavioral sensitization also surely involves more complex neuruadaptations than just an enhancement in dopamine release, although to date most studies of sensitization have focussed only on dopamine. There is already evidence that the induction of sensitization involves a different cellular site of drug action than the expression of sensitization ${ }^{160.269}$, that cross-sensitization does not occur between all addictive drugs ${ }^{160,269}$, that an action of drugs on neural systems other than dopamine is required to induce sensitization $^{54,161,164}$ and that there are sensitization-related changes in other neurotransmitter systems ${ }^{122,235,313,314}$. Similarly, Stewart ${ }^{323}$ has argued that the conditioned stimulus control of sensitization may occur at different synaptic sites, depending on the specific actions of different drugs. All of this suggests 
that sensitization involves neuroadaptations at multiple sites and in multiple neurotransmitter systems.

Nevertheless, in our present state of ignorance it is a reasonable working hypothesis that the adaptations in dopamine systems described here, involving an enhancement in mesotelencephalic dopamine neurotransmission, form a critical link in a chain of events leading to drug craving in addicts. We readily acknowledge, however, that some addictive drugs could produce such incentive-sensitization effects by an action on other, as yet unidentified, neural systems, including, for example, output pathways from the ventral striatum to the ventral pallidum ${ }^{180}$. On the other hand, it is also possible that the dopamine-independent rewarding effects of some drug treatment regimens ${ }^{181}$ involve the activation of this same dopamine-incentive salience circuitry, but at a later stage, 'downstream' from dopamine neurons. Regardless, we want to emphasize that the Incentive-Sensitization Theory of Addiction does not require that the sole or even primary site of drug-induced neuroadaptations responsible for craving specifically be on dopamine neurons. If it is not, then our assignment of sensitization of incentive salience to dopamine would be incorrect. Nevertheless, the concept that drug craving develops because of sensitization of incentive salience could still be fundamentally correct, but it would be mediated by another, as yet unidentified neural substrate.

\subsection{Note 5. Tolerance to drug pleasure}

The magnitude of the decrease in the subjective pleasurable effects of drugs is illustrated as being relatively small in Fig. 3 because, although the development of tolerance to the euphoric effects of drugs is widely accepted in the clinical literature, there is actually very little objective evidence for this. The evidence that is usually cited is that addicts tend to gradually escalate their dosage with repeated drug use. "One of the most insidious aspects of drug abuse is the seemingly inexorable tendency for addicts to increase their drug consumption over time" (ref. 86, p. 81). But Falk et al $^{86}$ point out there is very little evidence linking escalation in dose with tolerance to the subjective pleasurable effects of drugs. They state: "It is commonly presumed that... as tolerance develops, more drug must be ingested to satiate the addict's need for the drug. It is fascinating that there is little experimental data relevant to this assumption and that which does exist does not support" (p. 81). Indeed, there are a number of reports that addicts continue to experience euphoria even after years of drug use 193,211,213. $^{2}$ Some studies have suggested there even may be an increase in the pleasurable effects of morphine in long-term addicts, because naive subjects usually rate the effects of morphine as unpleasant, whereas experienced users ('postaddicts') overwhelmingly rate morphine effects as pleasant ${ }^{193}$. This apparent increase in the subjective effects of morphine is probably not due, however, to sensitization to its euphoric effects, but to tolerance to its aversive effects; because 'postaddicts' also report a lower incidence of nausea and vomiting than naive subjects.

Why then do addicts typically escalate their dose? A possible alternative explanation to tolerance of euphoria is that addicts increase dose to achieve the more intense (and more desirable) subjective effects produced by larger doses. They are able to do this only because tolerance develops to the aversive 'side-effects' of drugs. That is, addicts increase their dose because they can, without the dire negative effects experienced by naive users. Doses that might be unpleasant or even life-threatening in inexperienced users, are 'tolerated' by experienced users, because of tolerance to many of the drug's negative effects, including effects on the autonomic nervous system.

On the other hand, short-term tolerance to the euphoric effects of drugs may play a role in the escalation of dose seen when drugs are administered in a 'run', as is often the case with amphetamine or cocaine. If a large supply of amphetamine or cocaine is readily available addicts often readminister the drug as soon as the effects of the previous dose begins to dissipate. However, if successive administrations are given too close together in time the positive effects of the drug may be 'masked' or suppressed by a transient depression of brain 'reward' systems ${ }^{105,174,204}$. As pointed out by Stewart ${ }^{324}$ : "Tolerance to the rewarding effects of opiates has becn found in cxperiments in which animals were exposed to the drugs continuously prior to place preference training ${ }^{299 "}$ "(also see ref. 81 ) and in humans there is a "rapid within-session development of tolerance to the subjective mood effects of cocaine, but this dissipated completely within $24 \mathrm{~h}^{97}$, (also see ref. 95). Therefore, it may be that dose is escalated within a run to overcome this apparent short-term tolerance to the pleasurable effects of the drug. But this may not be relevant to the escalation of dose seen over the long-term, that is, between runs.

In summary, although we indicate in Fig. 3 that some tolerance develops to the subjective pleasurable effects of drugs we are aware that this is a complicated and largely unresolved issue. But whether the subjective pleasurable effects show some tolerance or no change with repeated drug administration, the development of an addiction is still characterized by an increasing dissociation between 'wanting' drugs and 
'liking' drugs. As Fig. 3 illustrates, 'wanting' drugs, produced by the attribution of incentive salience to the act of drug taking and to drug-related stimuli and their mental representations, increases dramatically during addiction; 'wanting' evolves into craving. At the same time 'liking' drugs does not increase.

\subsection{Note 6. The compulsive nature of addictive behavior}

It is interesting to speculate that addictive behavior may be so compulsive in nature because the neuroadaptations underlying drug addiction are in some way related to other obsessive-compulsive disorders. It is well known that hyperactivity in dopamine systems results in behaviors with a highly stereotyped (compulsive?) structure. For example, relatively low doses of amphetamine or cocaine elicit high levels of locomotor activity and the pattern of locomotion is abnormally stereotyped $^{224,285}$. At higher doses locomotor activity is diminished as animals engage in highly stereotyped, narrowly focussed, repetitive behaviors ${ }^{254}$. Similar patterns of stereotyped behavior ('punding') have been described extensively in human amphetamine users ${ }^{254,286}$. A number of compulsive behavioral disorders have been linked to dysfunction in the striatum, including obsessive-compulsive disorder itself, Tourette's syndrome, tic disorders ${ }^{18,255,256}$ and Huntington's Disease (ref. 59 and N. Wexler, personal communication). Thus, there are a number of different disorders in which dysfunction in the striatum has been associated with compulsive, repetitive thoughts (obsessions) and actions (compulsions). It is interesting to speculate, therefore, that some of the neural changes underlying drug addiction may be, in some respects, similar to those responsible for other obsessive-compulsive disorders.

\subsection{Note 7. Benzodiazepines and sedative-hypnotics}

There are drugs of abuse that do not seem to fit this profile, namely the benzodiazepines (BZ) and barbiturates. These compounds have biphasic effects on behavior, producing mild psychomotor activation at low doses and a marked depression of motor activity at higher doses ${ }^{365}$, but they may not increase dopamine neurotransmission. Although the psychomotor-activating effects of a low dose of diazepam $(0.25 \mathrm{mg} / \mathrm{kg})$ has been reported to require the activation of dopamine systems $^{311}$, microdialysis studies have found that diazepam and midazolam decrease extracellular dopamine in the nucleus accumbens ${ }^{92,146}$ (although in these latter studics relatively high doses were used, $>0.5 \mathrm{mg} / \mathrm{kg}$ ). It may be that the Incentive-Sensitization Theory does not account for why these particular drugs are used recreationally. It should be noted, how- ever, the BZ's are not very addictive and in normal experimental subjects they have "little or virtually no reinforcing effects" (ref. 56, p. 142). They do not produce the compulsive pattern of drug-seeking and drug-taking behavior characteristic of amphetamine, cocaine or the opiates ${ }^{371}$. Whether the addictive potential of alcohol can he accounted for by Incentive-Sensitization remains to be seen. As cited in the text, there are reports that alcohol: (1) produces psychomotor activation, especially in alcohol-preferring strains; (2) increases extracellular dopamine; and (3) produces sensitization. But there have been very few studies, they are not all consistent and therefore, more work is needed to resolve the issue.

\subsection{Note 8. The role of dopamine in mediating the effects of conditioned incentives}

The view of how conditioned incentive stimuli evoke relapse proposed here is similar to that of Stewart et al. ${ }^{326}$, except we hypothesize drug-associated stimuli evoke craving by activation of a sensitized neural system that specifically mediates incentive salience. Like Stewart et al. ${ }^{326}$ we suggest that this neural system involves mesotelencephalic dopamine projections to the ventral striatum and that conditioned incentive stimuli act much like a 'priming' dose of a drug itself; producing a small increase in dopaminergic activity. The difference is that we specify the consequence of this enhanced dopamine activity to be conditioned incentive salience, not necessarily a conditioned affective state, a 'high'. We do not deny, however, that conditioned pleasure can be elicited seperately, presumably via associative activation of separate neural systems.

We need to acknowledge, however, that there has been relatively little research on the role of dopamine in mediating the effects of conditioned incentive stimuli, especially conditioned incentive stimuli established through their association with drugs. There is considerable evidence that activation of the ventral striatal dopamine system enhances responding for conditioned incentive stimuli established by pairing a neutral stimulus with a natural incentive, like food or water $^{44,127,168,169,262,333,334}$ (for reviews see refs. 85, 263). There is also a general consensus that dopamine systems are critical in the process by which stimuli acquire conditioned incentive properties through their association with natual incentives or drugs $^{2,20,21,32,74,77,113,129,219,222,344,352}$ (cf. refs. 207,260, 317). It has been suggested, however, that once acquired, conditioned incentive stimuli may activate behavior independently of dopamine. This conclusion is based on reports that dopamine receptor blockade with pimozide (or in one experiment, haloperidol) does not 
prevent the conditioned psychomotor activation evoked by a conditioned stimulus associated with food ${ }^{139}$, or drugs $^{20,21,352}$; although the absolute amount of psychomotor activation produced by a food-associated CS is reduced ${ }^{139}$. This idea is controversial, however, because others have reported that dopamine antagonism does attenuate the expression of conditioned 'preparatory behaviors' (including locomotor activity) produced by a conditioned stimulus signaling food ${ }^{31,32,33}$ (also see ref. 353), as well as conditioned responses established by drugs ${ }^{76,77,113,130,247}$. Also, under some conditions dopamine antagonists may induce gradual extinctionlike effects on conditioned responding ${ }^{360,364}$. Such effects may be interpreted by the hypothesis that low doses of dopamine antagonists impair the 'reboosting' of incentive salience to established conditioned stimuli, which could occur each time an incentive stimulus is encountered. This 'reboosting' may be essential for the maintainence of a conditioned response ${ }^{27}$.

Direct measures of dopamine neurotransmission also support the hypothesis that this neural system mediates the incentive effects of conditioned stimuli ${ }^{31}$. Conditioned stimuli predictive of food have been reported to increase the discharge rate of dopamine neurons ${ }^{183,199,287,289}$, to increase dopamine metabolism in the nucleus accumbens ${ }^{34}$ and to increase a chronoamperometric signal thought to reflect extracellular dopamine ${ }^{239}$. Similarly, conditioned stimuli associated with psychomotor stimulants or opiates are reported to enhance dopamine metabolism ${ }^{191,234,283}$, to elevate a dopamine-related electrochemical signal associated with cocaine ${ }^{115}$ and in the presence of the unconditioned stimulus, to elevate dopamine in dialysate ${ }^{252}$. On the other hand, negative results have been reported as well ${ }^{16,38,39,93.348}$ and thercfore, more work is needed to resolve the discrepancies.

\subsection{Note 9. Stress, aversive stimuli and stimulant-in- duced psychoses}

Not only do pleasant natural incentives, such as food, water and access to a mate activate mesotelencephalic dopamine systems ${ }^{51,66,126,214,246,376}$, but so do some presumably unpleasant aversive events, including classical stressors. Stressors are particularly effective in activating dopamine projections to the medial frontal cortex and to the shell of the nucleus accumbens ${ }^{1,71,336}$. Intense aversive stimuli may also activate the nigrostriatal dopamine system ${ }^{1}$. Conditioned stressors (previously neutral stimuli paired with an aversive event) can activate mesotelencephalic dopamine systems as well, increasing dopamine metabolism in the frontal cortex ${ }^{125}$ and the concentration of dopamine in nucleus accumbens dialysate ${ }^{377}$. What does this mean for the hypoth- esis that dopamine mediates incentive salience and that dopaminergic activation makes stimuli salient, attractive and 'wanted'?

There are at least two alternative explanations that can reconcile stress-induced activation of dopamine and the incentive-sensitization hypothesis and until further data are available the incentive salience hypothesis does not commit to either one. First, it is possible that mesotelencephalic dopamine systems mediate the salience of stimuli that signal unpleasant consequences as well as those that signal pleasant ones. The rustling noise that signals an approaching tiger should grab the attention no less than the sight of delectable food. The salience of both tiger and food may be mediated by dopamine systems, whereas the valence of that salience (attractive incentive vs. frightening warning) may be determined by the coactivation of other neural systems. A second possibility is that moderate levels of dopamine activation, such as that produced by natural incentives and stressors (see above for references), always makes stimuli attractively salient, whereas even higher levels of dopamine activation makes stimuli frightening.

Is it non-sensical to say that stress can make stimuli more attractive? Not at all. Stressors are known to potentiate behavior that is ordinarily incentive-based. For example, stress-induced feeding is a phenomenon that has been well documented in both animals and humans $s^{10,223}$. Stress may cause mesotelencephalic dopamine systems to magnify the incentive salience attributed to known incentives such as familiar foods, thus leading to increased eating. Furthermore, stressors themselves may sometimes fascinate and elicit approach, rather than drive an individual away. For example, in a laboratory model of predator mobbing rats will repeatedly approach an electrified object that has in the past given them a shock and will attempt to bury the offending object by pushing sand, etc. upon $\mathrm{it}^{245}$. Also, under particular conditions animals will work (lever press) to deliver electric shocks to themselves, shocks that are known to be otherwise aversive (ref. 86 for review). Indeed, rats will even bar press to self-administer corticosterone i.v., in doses that produce a plasma concentration comparable to that seen during mild stress and this is associated with an increase in nucleus accumbens dopamine neurotransmis$\operatorname{sion}^{244}$. Furthermore, animals that are more prone to aequire a drug self-administration habit are more sensitive to the reinforcing effects of corticosterone than 'low risk' animals. Corticosterone also produces a larger increase in dopamine neurotransmission in 'high risk' animals than in 'low risk' animals ${ }^{244}$. These examples suggest that stress-induced dopamine activation may 
indeed activate incentive processes - quite separately from their activation of pain, aversion or discomfort. Which of these alternatives best describes the role of dopaminergic salience attribution during stress and in response to aversive stimuli will require further research to resolve.

In the examples above we suggest that the moderate level of dopamine activation produced by natural incentives and even stressors, may increase incentive salience. Likewise, we posit that a moderate level of dopamine activation produced by addictive drugs enhances incentive salience and the higher levels of dopamine activation produced by increasing doses of addictive drugs may progressively increase incentive salience. The ability of addictive drugs to elevate dopamine neurotransmission beyond that which normally occurs may be the feature of drugs that make them such potent incentives. However, this may be true only up to a point. Exceedingly high levels of dopamine activation may sometimes result in markedly aversive experiences. Although the attribution of incentive salience can make stimuli in the environment 'brighter' and more attractive, beyond a certain point the world may become too 'bright': stimuli may become confusing, distracting and potentially frightening. For example, the hallucinations and terror of amphetamine psychosis may reflect the excessive and indiscriminate attribution of salience to all stimuli in general, by a wildly hyperfunctioning dopamine system. The sensitization of dopamine neurotransmission may explain why the propensity to amphetamine or cocaine psychosis usually develops in a progressive, sensitization-like fashion and why the susceptibility to stimulant-induced (or stress-induced) psychosis persists for years after the discontinuation of drug use ${ }^{279,280}$.

The symptoms of stimulant-induced psychosis are very similar to those seen in paranoid schizophrenia ${ }^{292}$ and the suggestion that they are due to wildly excessive incentive salience is consistent with some current hypotheses regarding the nature of schizophrenia. It has been suggested" 331 , for example, that, "mesolimbic dopamine activation may regulate the extent to which particular types of environmental cues elicit or shape appetitive behavior"... and a loss of this 'gating' function by the overactivation of dopamine systems "may result in cognitive 'flooding', information overload and cognitive fragmentation in clinical states putatively associated with dopamine overactivity [such as schizo-

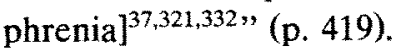

\subsection{Note 10. Dopamine antagonists and therapy}

At first sight, it might appcar that an implication of the Incentive-Sensitization Theory is that an effective treatment for excessive craving would be to block dopamine receptors with a postsynaptic antagonist such as pimozide or haloperidol. However, it is not at all clear that such a treatment would be effective. In fact, there are several reasons for doubting the usefulness of dopamine antagonists as a treatment for addiction.

Even though the Incentive-Sensitization Theory proposes that excessive craving for drugs results directly from sensitization of dopamine neurotransmission, dopamine antagonists may not be as useful in reducing the expression of pre-established incentives (attribution of incentive salience that is directed by existing associations) as they are in blocking the acquisition of new incentives (attribution of incentive salience to previously neutral stimuli). An addict is already too late for a treatment that blocks the acquisition of incentive sensitization to work. Only a treatment that blocked the expression of sensitized incentive salience would be helpful.

A large body of evidence from animal studies of dopamine antagonist effects on incentive motivation indicates that while the acquisition of, for example, a conditioned preference for an environment paired with drug administration is nearly always blocked by a dopamine antagonist, the behavioral expression of a preference that was previously conditioned is only sometimes suppressed by the same drug (see the aforementioned Note 8 ). There are a number of possible explanations for the equivocal effects of dopamine antagonists on the expression of pre-existing incentives. First, it might be that dopamine antagonists actually do reduce the incentive salience of drug-paired conditioned stimuli, but that they also reduce the incentive salience of all other stimuli. This would produce an absolute reduction of all incentive motivation but would leave the relative incentive value of stimuli unchanged (ref. 139 for example). Although an addict might crave drugs less after taking a dopamine antagonist, drugs would still be wanted more than anything else and drug seeking would still dominate behavior. Second, it might be that the neural processes that mediate the expression of sensitized incentive salience truly are more resistant to the effects of dopamine antagonists than are the neural processes which mediate the establishment of sensitization. If so, only very high doses of neuroleptics, which might seriously disrupt many aspects of normal behavior, would be sufficient to suppress drug craving. We do not know why this should be true, but there are a number of possible mechanisms that could explain it. For example, once established sensitization might be associated with neuroadaptations that extend to systems 'downstream' from dopamine neurons themselves. These neural systems 
may continue to respond excessively even if dopamine neurotransmission is reduced. On the other hand, the acquisition of sensitization may depend more specifically upon dopamine activation alone.

Another possibility is that the acquisition vs. expression of sensitization are mediated by different dopamine subsystems that have, for example, different dopamine receptor subtypes, etc. Hiroi and White ${ }^{130}$ noted that when a dopamine receptor antagonist failed to selectively decrease the expression of conditioned responding (see the aforementioned Note 8) "neuroleptics with a higher affinity for D2 than D1 receptors", were used and further noted that "a D1 antagonist, $\mathrm{SCH} 23390$ is equally effective in blocking acquisition and expression of an amphetamine conditioned place preference, whereas much higher doses of D2 antagonists are required to block expression than acquisition. Thus, some workers may have failed to observe blocking of the expression of learned behaviors because they used an inappropriate dose range of D2 antagonists" (pp. 40,41).

Finally, even if neuroleptic drugs were effective at suppressing drug craving at moderate doses, their usefulness could be compromised by the possibility that addicts would refuse to take them. Anecdotal evidence abounds to suggest that neuroleptics are unpopular drugs among patients who take them. Aside from their potential motor effects, it is not surprising that this should be so. By the incentive salience hypothesis, a drug that directly suppressed the attribution of incentive salience would make the world 'less bright'. Even though the neural substrates of pleasure would not be suppressed, such a drug could produce 'sham anhedonia' - that is, the conscious inference by the addict that pleasure was reduced via cognitive interpretation ("I don't want anything very much, therefore I must not like anything") - just as direct activation of incentive salience should produce 'sham reward'27.

\section{GLOSSARY}

Addiction. There has been considerable debate regarding the appropriate definition of drug addiction. We will use the term here in the sense proposed by a World Health Organization Expert Committee in $1981^{79,148}$. Drug addiction is defined as "a syndrome in which the use of a drug is given a much higher priority than other behaviors that once had higher value."... "In its extreme form [addiction] is associated with compulsive drug-using behavior and it exhibits the characteristics of a chronic relapsing disorder" (ref. 148, p. 522). The phrase 'addictive behavior' is used to refer collectively to obsessive drug craving and to compulsive drug-seeking and drug-taking behavior.

Addictive behavior. (see Addiction)

Appetitive motivation. (see Incentive motivation)

Aversion. The subjective experience of a sensation as actively unpleasant or the underlying evaluative processes and neural mechanisms that directly produce this subjective experience. The opposite of pleasure or euphoria (see Pleasure). Aversion results from an active evaluation of a sensation carried out by brain systems. In the context of addiction, aversion can be synonymous with the symptoms associated with drug withdrawal, including physical distress and dysphoria. Aversion can also refer to direct subjective effects produced by a drug that can be discriminated from pleasure by the user.

Conditioned incentive stimuli. (see Incentives)

Craving and 'Wanting'. These are used in accordance with their usual English meaning, which for 'wanting' refers to the subjective experience of needing or desiring something ("to feel a need or desire for"; Random House Dictionary of the English Language, 2nd edn., 1987). We further propose, however, that this experience is produced by the psychological process of salience attribution (see incentive salience), that is, the attribution of incentive salience to an external event or its mental representation. The process of incentive salicnce attribution is pre-concious and only the result of this psychological process is accessible to consciousness. When this occurs it is interpreted ${ }^{228}$ as a subjective feeling of 'wanting'. For our purposes craving and 'wanting' differ only in magnitude: craving equals intense 'wanting'184. In the addict, craving is the experience associated with excessive incentive salience, which results from drug-induced sensitization of the neural systems that attribute salience to incentives.

Dependence, drug. (see Addiction)

Euphoria. (see Pleasure)

Hedonics. (see Pleasure)

Incentive motivation(al). A psychological theory (also see Fig. 2) of how goal-direction is controlled by the stimulus properties of the target ${ }^{30,339}$. Incentive motivation is one of a number of potential psychological mechanisms for controlling the direction of motivated behavior. Drive reduction and opponent processes are examples of other potential mechanisms that might control behavior independently of incentive processes (see Toates ${ }^{339}$ for discussion and evidence).

Incentive motivation appears to be the chief mechanism that controls behavior directed towards natural incentives such as food, water and a potential mate and towards more artificial incentives, such as self-administered drugs and reinforcing electrical brain stimula- 
tion ${ }^{30.339}$. In the context of addiction, incentive motivation and appetitive motivation are synonymous: that is, appetitive motivation works primarily through incentive processes ${ }^{339}$. Incentive motivation directed towards particular stimuli results from the outcome of a threestage process. First, the neural substrates for pleasure are activated by the consequences of a particular act or event. Second, pleasure is associated with the object, act, event or place in which pleasure occurs by the processes of classical associative learning. Third, salience is attributed to subsequent perceptions and mental representations of the associated object, act, event or place, by a separate neural system from those responsible for the first two processes. This third process of salience attribution (incentive salience) is proposed here to involve dopamine. The attribution of incentive salience causes the associated situation to become attractive and 'wanted' and it is this psychological process that produces the direct manifestation of incentive motivation: goal-directed seeking and instrumental behavior.

Incentive salience. Refers to the attractiveness of external stimuli, events, places and their mental representations; their ability to capture attention (also see Fig. 2). The term, incentive salience, applies always to the perception of external events and to internal representations of those events. Incentive salience must be actively generated (attributed) by the brain and assigned to particular perceptions and representations, based on their association with past activation of mesotelencephalic dopamine systems. For any given stimulus incentive salience will vary at different times depending upon changes in learned associations regarding the stimulus, the internal state of the perceiver and most specifically, the degree of activation of the dopamine systems that mediate incentive salience. Incentive salience is one of a number of psychological mechanisms that can produce direct behavior (other mechanisms include drive reduction and goal-directed computational algorithms that do not depend on modulated perception of the goal; see Toates ${ }^{339}$ ). Incentive salience constitutes one component of the complex process of incentive motivation. Although the assignment of incentive salience to an event normally is triggered by a pleasurable experience, manipulations of dopamine systems can disconnect incentive salience from pleasure and alter incentive salience independently. It is hypothesized that the attribution of incentive salience to an event or representation is an unconscious process; only the product of this process, the perception of the object as 'wanted', is interpreted and consciously experienced.
Incentive stimuli (incentives). Stimuli that have been attributed with incentive salience. The perception and mental representation of these stimuli are transformed as a consequence and as incentive stimuli they become salient, attractive, 'wanted', and approached. The terms natural incentives and artificial incentives are also used. The 3-stage process described for incentive motivation evolved to enable animals and humans to recognize and respond to 'natural' incentives. By natural incentives we mean stimuli such as food, water, social and scxual partners, thermal and tactile sensations, which have been endowed by evolution with the capacity to elicit pleasure and incentive salience under particular conditions (e.g., under certain hormonal conditions). Most natural incentives exert their effects via sensory receptors. 'Artificial' incentives, on the other hand, such as addictive drugs or electrical brain stimulation, bypass sensory receptors and activate the component processes of incentive motivation more directly. Most incentive stimuli are conditioned incentive stimuli: stimuli that have become incentives as a consequence of associative learning during the three-stage process described for incentive motivation. Conditioned incentive stimuli not only include the arbitrary lights and sounds used in laboratory experiments (e.g., an auditory tone that signals food delivery), but also the stimulus configurations that must be learned through experience that allow natural incentives to be recognized (e.g., the sight of a delectable food; the sound of a loved one's voice). Conditioned incentive stimuli are often referred to as conditioned rewards (see below), secondary rewards or secondary reinforcers (see below). 'Liking'. See Pleasure.

Negative reinforcement. See Reinforcement).

Pleasure and 'liking'. These are used in accordance with their usual English meaning, which refers to the subjective experience of a sensation as pleasurable or hedonic and the underlying evaluative and neural processes that directly produce this subjective experience. The opposite of aversion (see above), Pleasure is usually the first stage of the larger process of incentive motivation (together with incentive salience and associative learning) and serves as the normal trigger that activates components of associative learning and incentive salience. By itself, however, pleasure is not equivalent to either reward or 'wanting': it is merely a subjective experience or feeling. The evaluation of the sensation that produces pleasure is pre-conscious; only the product, the subjective pleasure, is experienced. In the present paper and in the context of addiction, the term pleasure is used synonymously with the terms euphoria, hedonia or positive affective state. 
Positive affective state. (see Pleasure).

Positive reinforcement. (see Reinforcement).

Reinforcement. A purely behavioral and descriptive term for the relationship between the occurrence of a stimulus and changes in the subsequent probability of the behavior that preceded it. Reinforcement denotes a change in the probability of a behavior (increased or decreased) that is contingent on presentation of stimuli. Reinforcement does not offer either a psychological explanation or a physiological explanation of why the probability of a behavior is changed; it merely notes the existence of the change. Reinforcement can be positive or negative. Positive reinforcement refers to increases in the probability of emission of a behavior produced by subsequent presentation of a stimulus (the positive reinforcer). In the context of addiction the term positive reinforcement is sometimes used both in its proper descriptive sense and sometimes in the theoretical or explanatory sense of reward (see below), where the pleasure produced by a drug is implicitly assumed as a psychological explanation for the change in behavior. In order to avoid ambiguity in the present paper we use positive reinforcement only in its proper descriptive sense. Negative reinforcement refers to increases in the probability of emission of a behavior produced by subsequent omission or termination of a stimulus. For example, in the context of addiction, drugs may act as negative rcinforcers by relieving the distress of drug withdrawal.

Reward. The word reward is used in the literature in many different ways and for the most part we avoid the term. For us, the process of reward is essentially equivalent to the process of incentive motivation; that is, reward refers to the process of creating incentives (or, as a noun, a stimulus that triggers this process). Rewards (or incentives) cause future behavior to be changed in a goal-directed fashion so as to obtain again the situation or stimulus that triggered the process. This process normally requires three separate stages. The first stage is the activation of pleasure by the consequences of a particular act or event. In the second stage pleasure is associated with a mental representation of the object, act, event or place in which pleasure occurred, by the process of classical (associative) conditioning. The third stage involves the attribution of incentive salience to subsequent perceptions and representations of the associated object, act, event or place, which causes them to become 'wanted'. Stimuli that signal the availability of the incentive become attractive. Acts that led to the situation in the past are likely to be repeated. New acts, which the animal or person can predict (cognitively) will lead to the incen- tive in the future, are likely to be produced. If the three stages of normal reward (pleasure, associative learning, incentive salience) are separated, the process remains incomplete. Some separations have been achieved by brain manipulations; others are useful simply as illustrative 'thought experiments'. If the first stage of pleasure is activated without associative learning or salience attribution, then it is merely an isolated hedonic experience that remains unconnected to other events in the world or to subsequent behavior. If the first two stages occur alone so that pleasure is activated in conjunction with associative learning only, then associative conditioning of pleasure will occur to the associated events, but they will not be attributed with incentive salience. The events will become 'liked', but they will not be 'wanted' (this separation may possibly be achieved by destruction of brain dopamine systems ${ }^{28}$ ). Conversely, if the third stage, incentive salience, is activated alone, then 'wanting' arises in isolation from other processes. This may be achieved, for example, by a stimulating electrode that directly activates brain dopamine systems ${ }^{27}$. If salience attribution is magnified abnormally in conjunction with associative learning, but pleasure is not, for example, by the process of drug-induced sensitization discussed here, then the attribution of incentive salience becomes intensified and focused narrowly on the stimuli and acts associated with drug administration and they become patholugically 'wanted' (craved). This may be considered a type of 'sham reward', which shapes instrumental behavior and creates craving, but is dissociated from pleasure. Viewed from the outside, the behavior produced by sham reward and natural reward is identical on all measures of 'wanting' or instrumental performance. Only behavioral measures that are specifically sensitive to pleasure - rather than wanting - will identify sham reward (without pleasure) as distinct from natural reward (triggered by pleasure).

Salience. A salient stimulus is a stimulus that has been (or is being) attributed with incentive salience (see above). Salience refers to the feature(s) of the percept or representation of a stimulus that makes it highly noticcable and difficult to ignore. A salient stimulus is not merely more obvious, but it also becomes 'wanted' and attractive because, according to our usage, it is incentive salience that is attributed to the percept or mental representation (but see Note 9 in Ch. 6 on aversive salience).

Salience attribution. (see Incentive salience; Incentive motivation).

Secondary reinforcers. (see Incentive stimuli)

'Wanting'. (see Craving/'wanting'). 
Acknowledgements. Preparation of this manuscript and research by the authors was supported by Grants from the National Institute on Drug Abuse (04294) to T.E.R. and from the National Institutes of Health to K.C.B. (NS23959). An earlier version of this theory was first presented at a UCLA/NIDA conference on The Biological Basis of Substance Abuse, Santa Monica, CA, January 9-12, 1991. We are grateful to Aldo Badiani, Jill Becker, Craig Berridge, Barry Everitt, Tim Schallert, Jane Stewart, Frederick Toates, Keith Truiillo, Elliot Valenstein, Derek van der Kooy, Ian Whishaw and Roy Wise for their helpful and thought-provoking comments on an earlier draft of this paper

\section{REFERENCES}

1 Abercrombie, E.D., Keefe, K.A., Di Frischia, D.S. and Zigmond, M.J., Differential effect of stress on in vivo dopamine release in striatum, nucleus accumbens and medial frontal cortex, J. Neurochem., 52 (1989) 1655-1658.

2 Acquas, E., Carboni, E., Leone, P. and Di Chiara, G., SCH 23390 blocks drug-conditioned place-preference and place-aversion: anhedonia (lack of reward) or apathy (lack of mativation) after dopamine-receptor blockade?, Psychopharmacology, 99 (1989) $151-155$

3 Aizenstein, M.L., Segal, D.S. and Kuczenski, R., Repeated amphetamine and fencamfamine: sensitization and reciprocal cross-sensitization, Neuropsychopharmacology, 3 (1990) 283-290.

4 Akimoto, K., Hamamura, T., Kazahaya, Y., Akiyama, K. and Otsuki, S., Enhanced extracellular dopamine level may be the fundamental neuropharmacological basis of cross-behavioral sensitization between methamphetamine and cocaine - an in vivo dialysis study in freely moving rats, Brain Res., 507 (1990) $344-346$.

5 Akimoto, K., Hamamura, T. and Otsuki, S., Subchronic cocaine treatment enhances cocaine-induced dopamine efflux, studied by in vivo intracerebral dialysis, Brain Res, 490 (1989) 339-344.

6 Akiyama, K., Hamamura, T., Ujike, H., Kanzaki, A. and Otsuki, S., Methamphetamine psychosis as a model of relapse of schizophrenia - a behavioral and biochemical study in the animal model. In T. Nakazawa (Ed.), Taniguch Symposia on Brain Sciences, Vol. 14, Biological Basis of Schizophrenia, Japan Scientific Societies Press, Tokyo, 1991, pp. 169-184.

7 Anonymous, Alcoholics Anonymous, Alcoholics Anonymous World Services, New York, 1976, 3rd edn.

8 Antelman, S.M. and Chiodo, L.A., Amphetamine as a stressor. In 1. Creese (Ed.), Stimulants; Neurochemical, Behovioral and Clinical Perspectives, Raven Press, New York, 1983, pp. 269-299.

9 Antelman, S.M. Eichter, AJ., Black, C.A. and Kocan, D., Interchangeability of stress and amphetamine in sensitization, Science, 207 (1980) 329-331.

10 Antelman, S.M. and Szechtman, H., Tail pinch induces eating in sated rats which appears to depend on nigrostriatal dopamine, Science, 189 (1975) 731-733.

11 Babbini, M. and Davis, W.M., Time-dose relationships for locomotor activity effects of morphine after acute or repeated treatment, Br. J. Pharmacol, 46 (1972) 213-224.

12 Babbini, M., Gaiardi, M. and Bartoletti, M., Persistence of chronic morphine effects upon activity in rats 8 months after ceasing the treatment, Neuropharmacology, 14 (1975) 611-6114.

13 Bailey, C.S., Hsiao, S. and King, J.E. Hedonic reactivity to sucrose in rats: modification by pimozide, Physiol. Behav., 38 (1986) $447-452$.

14 Baker, H., Joh, T.H. and Reis, D.J., Genetic control of number of midbrain dopaminergic neurons in inbred strains of mice: relationship to size and neuronal density of the striatum, Proc. Natl. Acad. Sci. USA, 77 (1980) 4369-4373.

15 Barnett, J.V., Segal, D.S. and Kuczenski, R., Repeated amphetamine pretreatment alters the responsiveness of striatal dopamine-stimulated adenylate cyclase to amphetamine-induced desensitization, I. Pharmacol. Exp. Ther., 242 (1987) 40-47.

16 Barr, G.A., Sharpless, N.S., Cooper, S., Schiff, S.R., Paredes, W. and Bridger, W.H., Classical conditioning, decay and extinction of cocaine-induced hyperactivity and stereotypy, Life Sci., 33 (1983) 1341-1351.

17 Bartoletti, M., Gaiardi, M., Gubellini, G., Bacchi, A. and Babbini, M., Long-term sensitization to the excitatory effects of morphine. A motility study in post-dependent rats, Neuropharmacology, 22 (1983) 1193-1196.

18 Baxter, L.R.J., Schwartz, J.M., Bergman, K.S., Szuba, M.P., Guze, B.H., Mazziotta, I.C., Alazraki, A., Selin, C.E., Ferng, H.K, Munford, P., et al, Caudate glucose metabolic rate changes with both drug and behavior therapy for obsessive-compulsive disorder, Arch. Gen. Psychiary, 49 (1992) 681-689.

19 Beninger, R.J., The role of dopamine in locomotor activity and learning, Brain Res. Rev, 287 (1983) 173-196.

20 Beninger, R.J. and Hahn, B.L., Pimozide blocks establishment but not expression of amphetamine-produced environmentspecific conditioning, Science, 220 (1983) 1304-1306.

21 Beninger, R.J. and Herz, R.S., Pimozide blocks establishment but not expression of cocaine-produced environment-specific conditioning, Life Sci., 38 (1986) 1425-1431.

22 Beninger, R.J., MacLennan, A.J. and Pinel, J.P., The use of conditioned defensive burying to test the the effects of pimozide on associative learning, Pharmacol. Biochem. Behav., 12 (1980) $445-448$

23 Beninger, R.J., Mason, S.T., Phillips, A.G. and Fibiger, H.C.s The use of conditioned suppression to evaluate the nature of neuroleptic-induced avoidance deficits, J. Pharmacol. Exp. Ther., 213 (1980) 623-627.

24 Benjamin, D., Grant, E.R., Goldstein, K.R. and Pohorecky, L.A., Sensitization to the dopamine release-enhancing effects of ethanol demonstrated in male long-evans rats, Soc. Neurosi. Abstr, 18 (1992) 1431.

25 Benwell, M.E.M. and Balfour, D.J.K., The effects of acute and repeated nicotine treatment on nucleus accumbens dopamine and locomotor activity, Br. J. Phamacol, 105 (1992) 849-856.

26 Berridge, K.C., Modulation of taste affect by hunger, caloric satiety and sensory-specific satiety in the rat, Appetite, 16 (1991) 103-120.

27 Berridge, K.C. and Valenstein, E.S., What psychological process mediates feeding evoked by electrical stimulation of the lateral hypothalamus?, Behav. Neurosci., 105 (1991) 3-14.

28 Berridge, K.C., Venier, I.L. and Robinson, T.E., Taste reactivity analysis of 6-hydroxydopamine-induced aphagia: implications for arousal and anhedonia hypotheses of dopamine function, $B e$ hav. Neurosci., 103 (1989) 36-45.

29 Bijerot, W., Addiction to pleasure: a biological and social-psychological theory of addiction. In D.J. Lettieri, M. Sayers and H.W. Pearson (Eds), Theories on Drug Abuse: Selected Contemporary Perspectives, National Institute on Drug Abuse, Rockille, 1980 , pp. $246-255$.

30 Bindra, D., How adaptive behavior is produced: a perceptualmotivation alternative to response reinforcement, Behav. Brain Sci., 1 (1978) 41-91.

31 Blackburn, J.R., Pfaus, J.G. and Phillips, A.G., Dopamine functions in appetitive and defensive behaviours, Prog. Neurobiol, 39 (1992) $247-279$.

32 Blackburn, J.R., Phillips, A.G. and Fibiger, H.C., Dopamine and preparatory behavior: I. Effects of pimozide, Behav. Neurosci., 101 (1987) $352-360$.

33 Blackburn, J.R., Phillips, A.G. and Fibiger, H.C., Dopamine and preparatory behavior: III. Effects of metoclopramide and thioridazine, Behav. Neurosci, 103 (1989) 903-906.

34 Blackburn, J.R, Phillips, A.G., Jakubovic, A. and Fibiger, H.C., Dopamine and preparatory behavior: II. A neurochemical analysis, Behau. Neurosci., 103 (1989) 15-23.

35 Borison, R.L., Havdala, H.S. and Diamond, B.I., Chronic phenylethylamine stereotypy in rats: a new animal model for schizophrenia?, Life Sci., 21 (1977) 117-122.

36 Bradberry, C.W., Gruen, R.J., Berridge, C.W. and Roth, R.H., Individual differences in behavioral measures: correlations with mucleus accumbens dopamine measured by microdialysis, Pharmacol. Biochem. Behav, 39 (1991) 877-882.

37 Braff, D.L. and Geyer, M.A., Sensorimotor gating and the 
neurobiology of schizophrenia: human and animal model studies. In S.C. Schultz and C.A. Tamminga (Eds.), Schizophrenia: Scientific Progress, Oxford University Press, New York, 1989, pp. 124-136.

38 Brown, E.E. and Fibiger, H.C., Cocaine-induced conditioned locomotion: absence of associated increase in dopamine release, Neuroscience, 48 (1992) 621-629.

39 Brown, E.E., Robertson, G.S. and Fibiger, H.C., Evidence for conditional neuronal activation following exposure to a cocainepaired environment: role of forebrain limbic structures, $\mathrm{J}$. Neurosci., 12 (1992) 4112-4121.

40 Browne, R.G. and Segal, D.S., Metabolic and experimental factors in the behavioral response to repeated amphetamine, Pharmacol. Biochem. Behav., 6 (1977) 545-552.

41 Cabanac, M., Physiological role of pleasure, Science, 173 (1971) 1103-1107.

42 Cabanac, M., Sensory pleasure, Q. Reu. Biol, 54 (1979) 1-29.

43 Cabanac, M. and Lafrance, L., Postingestive alliesthesia: the rat tells the same story, Physiol. Behav., 47 (1990) 539-543.

44 Cador, M., Taylor, J.R. and Robbins, T.W., Potentiation of the effects of reward-related stimuli by dopaminergic-dependent mechanisms in the nucleus accumbens, Psychopharmacology, 104 (1991) 377-385

45 Camp, D.M. and Robinson, T.E., Susceptibility to sensitization. I. Sex differences in the enduring effects of chronic $D$ amphetamine treatment on locomotion, stereotyped behavior and brain monoamines, Behav. Brain Res., 30 (1988) 55-68.

46 Camp, D.M. and Robinson, T.E., Susceptibility to sensitization. II. The influence of gonadal hormones on enduring changes in brain monoamines and behavior produced by the repeated administration of D-amphetamine or restraint stress, Behav. Brain Res. 30 (1988) 69-88.

47 Carison, J.N. and Glick, S.D., Cerebral lateralization as a source of interindividual differences in behavior, Experientia, 45 (1989) $788-798$.

48 Castañeda, E., Becker, J.B. and Robinson, T.E., The long-term effects of repeated amphetamine treatment in vivo on amphetamine, $\mathrm{KCl}$ and electrical stimulation evoked striatal dopamine release in vitro, Life Sci., 42 (1988) 2447-2456.

49 Childress, A.R., Ehrman, R., McLellan, A.T., MacRac, J., Natale, M. and O'Brien, C.P., Negative mood states trigger conditioned drug craving and conditioned withdrawal in opiate abuse patients, J. Subst. Abuse Treat, in press.

50 Childress, A.R., McLellan, A.T., Ehrman, R. and O'Brien, C.P., Classically conditioned responses in opioid and cocaine dependence: a role in relapse?, NIDA Res. Monogr., 84 (1988) 25-43.

51 Church, W.H., Justice Jr., J.B. and Neill, D.B., Detecting behaviorally relevant changes in extracellular dopamine with microdialysis, Brain Res., 412 (1987) 397-399.

52 Clarke, P.B.S., Mesolimbic dopamine activation - the key to nicotine reinforcement? In Ciba Foundation Symposium 152; The Biology of Nicotine Dependence, Wiley, Chichester, 1990, pp. 153-168.

53 Clifton, P.G., Rusk, I.N. and Cooper, S.J., Stimulation and inhibition of food intake by the selective dopamine D2 agonist, N-0437: a meal pattern analysis, Pharmacol. Biochem. Behav., 33 (1989) $21-26$

54 Cole, B.J., Cador, M., Stinus, L., Rivier, C., Rivier, J., Vale, W., Le Moal, M. and Koob, G.F., Critical role of the hypothalamic pituitary adrenal axis in amphetamine-induced sensitization of behavior, Life Sci, 47 (1990) 1715-1720.

55 Cole, B.J., Cador, M., Stinus, L., Rivier, J., Vale, W., Koob, G.F. and Le Moal, M., Central administration of a CRF antagonist blocks the development of stress-induced behavioral sensitization, Brain Res., 512 (1990) 343-346.

56 Cole, J.O. and Chiarello, R.J., The benzodiazepines as drugs of abuse, J. Psychiat. Res., 24 (1990) 135-144.

57 Cools, A.R., Differential role of mineralocorticoid and glucocorticoid receptors in the genesis of dexamphetamine-induced sensitization of mesolimbic, alpha 1 adrenergic receptors in the ventral striatum, Neuroscience, 43 (1991) 419-428.

58 Crabbe, J.C., Johnson, N.A., Gray, D.K., Kosobud, A. and
Young, E.R., Biphasic effects of ethanol on open-field activity: sensitivity and tolerance in $\mathrm{C} 57 \mathrm{BL} / 6 \mathrm{~N}$ and $\mathrm{DBA} / 2 \mathrm{~N}$ mice, $J$ Comp. Physiol Psychol, 96 (1982) 440-451.

59 Cummings, J.L. and Cunningham, K., Obsessive-compulsive disorder in Huntington's disease, Biol. Psychiatry, 31 (1992) 263270.

60 Cunningham, C.L., Niehus, D.R., Malntt, D.H. and Prather, L.K. Genetic differences in the rewarding and activating effects of morphine and ethanol, Psychopharmacology, 107 (1992) 385393.

61 Cunningham, C.L. and Noble, D., Conditioned activation induced by ethanol: role in sensitization and conditioned place preference, Pharmacol. Biochem. Behav., 43 (1992) 307-313.

62 Cunningham, S.T. and Kelley, A.E., Evidence for opiate-dopamine cross-sensitization in the nucleus accumbens: studies of conditioned reward, Brain Res. Bull., 29 (1992) 675-680.

63 Dackis, C.A. and Gold, M.S., Bromocriptine as a treatment of cocaine abuse, The Lancet, May (1985) 1151-1152.

64 Dackis, C.A. and Gold, M.S., New concepts in cocaine addiction: the dopamine depletion hypothesis, Neurosci. Biobehav. Rev., 9 (1985) 469--477.

65 Damsma, G., Day, J. and Fibiger, H.C., Lack of tolerance to nicotine-induced dopamine release in the nucleus accumbens, Eur. J. Pharmacol, 168 (1989) 363-368.

66 Damsma, G., Pfaus, J.G., Wenkstern, D., Phillips, A.G. and Fibiger, H.C., Sexual behavior increases dopamine transmission in the nucleus accumbens and striatum of male rats: comparison with novelty and locomotion, Behav. Neurosci., 106 (1992) 181191.

67 Davis, J.D., The microstructure of ingestive behavior, Ann. NY Acad. Sci, 575 (1989) 106-119.

68 Deminiere, J.M., Piazza, P.V., Guegan, G., Abrous, N., Maccari, S., Le Moal, M. and Simon, H., Increased locomotor response to novelty and propensity to intravenous amphetamine self-administration in adult offspring of stressed mothers, Brain Res., 586 (1992) 135-139.

69 Deroche, V., Piazza, P.V., Casolini, P., Maccari, S., Le Moal, M. and Simon, H., Stress-induced sensitization to amphetamine and morphine psychomotor effects depend on stress-induced corticosterone secretion, Brain Res., 598 (1992) 343-348.

70 Deroche, V., Piazza, P.V., Maccari, S., Le Moal, M. and Simon, $\mathrm{H}$. Repeated corticosterone administration sensitizes the locomotor response to amphetamine, Brain Res., 584 (1992) 309-313.

71 Deutch, A.Y. and Cameron, D.S., Pharmacological characterization of dopamine systems in the nucleus accumbens core and shell, Neuroscience, 46 (1992) 49-56.

72 Dews, P., Remarks. In T. Thompson and K.R. Unna (Eds.) Predicting Dependence Liability of Stimulant and Depressant Drugs, University Park Press, Baltimore, 1977, pp. 75-79.

73 Di Chiara, G. and Imperato, A., Drugs abused by humans preferentially increase synaptic dopamine concentrations in the mesolimbic system of freely moving rats, Proc. Natl. Acad. Sci. USA, 85 (1988) 5274-5278.

74 Di Lullo, S.L. and Martin-Iverson, M.T., Evidence for presynaptic dopamine mechanisms underlying amphetamine-conditioned locomotion, Brain Res., 578 (1992) 161-167.

75 Doyle, T.J., Berridge, K.C and Gosnell, B.A., Morphine enhances taste pleasure, Pharmacol. Biochem. Behav, in press.

76 Drew, K.L. and Glick, S.D., Classical conditioning of amphetamine-induced lateralized and nonlateralized activity in rats, Psychopharmacology, 92 (1987) 52-57.

77 Drew, K.L. and Glick, S.D., Role of D-1 and D-2 receptor stimulation in sensitization to amphetamine-induced circling behavior and in expression and extinction of the Pavlovian conditioned response, Psychopharmacology, 101 (1990) 465-471.

78 Edmonds, D.E. and Gallistel, C.R., Reward versus performance in self-stimulation: electrode-specific effects of alpha-methyl-ptyrosine on reward in the rat, J. Comp. Physiol. Psychol., 91 (1977) 962-974.

79 Edwards, G., Nomenclature and classification of drug- and alcohol-related problems: a WHO memorandum, Bull. World Health Org., 59 (1981) 225-242. 
80 Ehrman, R., Ternes, J., O'Brien, C.P. and McLellan, A.T., Conditioned tolerance in human opiate addicts, Psychopharmacology, 108 (1992) 218-224.

81 Emmett-Oglesby, M.W. and Lane, J.D., Tolerance to the reinforcing effects of cocaine, Behav. Phamacol, 3 (1992) 193-200.

82 Ettenberg, A., Dopamine, neuroleptics and reinforced behavior, Neurosci. Biobehav. Rev., 13 (1989) 105-111.

83 Ettenberg, A., Haloperidol prevents the reinstatement of amphetamine-rewarded runway responding in rats, Pharmacol. Biochem. Behav., 36 (1990) 635-638.

84 Everitt, B.J., Sexual motivation: a neural and behavioural analysis of the mechanisms underlying appetitive and copulatory responses of male rats, Neurosci. Biobehav. Rev., 14 (1990) $217-232$.

85 Everitt, B.L. and Robbins, T.W., Amygdala-ventral striatal interactions and reward-related processes. In J.P. Aggleton (Ed.), The Amygdala: Neurobiological Aspects of Emotion. Memory, and Mental Dysfunction, Wiley-Liss, New York, 1992, pp. 401-429.

86 Falk, J.L., Dews, P.B. and Schuster, C.R., Commonalities in the environmental control of behavior. In P.K. Levisun, D.R. Gerstein and D.R. Maloff (Eds.), Commonalities in Substance Abuse and Habitual Behavior, D.C. Heath and Co., Lexington, MA, 1983 , pp. $47-110$.

87 Falk, J.L. and Feingold, D.A., Environmental and cultural factors in the behavioral action of drugs. In H.Y. Meltzer (Ed.), Psychopharmacology: The Third Generation of Progress, Raven Press, New York, 1987, pp. 1503-1510.

88 Falk, J.L., Ma, F. and Lau, C.E., Chronic oral cocaine self-administration: pharmacokinetics and effects on spontaneous and discriminative motor functions, J. Pharmacol. Exp. Ther., 257 (1991) 457-465.

89 Fibiger, H.C. and Phillips, A.G., Increased intracranial selfstimulation in rats after long-term administration of desipramine, Science, 214 (1981) 683-685.

90 Fibiger, H.C. and Phillips, A.G., Reward, motivation, cognition: psychobiology of mesotelencephalic dopamine systems. In Handbook of Physiology, Vol. IV, Intrinsic Regulatory Systems of the Brain, American Physiology Society, Bethesda, MD, 1986, pp. $647-675$

91 Fink, J.S. and Reis, D.J., Genetic variations in midbrain dopamine cell number: parallel with differences in responses to dopaminergic agonists and in naturalistic behaviors mediated by central dopaminergic systems, Brain Res, 222 (1981) 335-349.

92 Finlay, J.M., Damsma, G. and Fibiger, H.C., Benzodiazepine-induced decreases in extracellular concentrations of dopamine in the nucleus accumbens after acute and repeated administration, Psychopharmacology, 106 (1992) 202-208.

93 Finlay, J.M., Jakubovic, A., Phillips, A.G. and Fibiger, H.C., Fentanyl-induced conditional place preference: lack of associated conditional neurochemical events, Psychopharmacology, 96 (1988) 534-540.

94 Fischman, M.W., Relationship between self-reported drug effects and their reinforcing effects: studies with stimulant drugs, NIDA Res. Monogr., 92 (1989) 211-230.

95 Fischman, M.W. and Foltin, R.W., Self-administration of cocaine by humans: a laboratory perspective. In G.R. Bock and J. Whelan (Eds.), Cocaine: Scientific and Social Dimensions. CIBA Foundation Symposium No. 166, Wiley, Chichester, UK, 1992, pp. $165-180$.

96 Fischman, M.W., Foltin, R.W., Nestadt, G. and Pearlson, G.D. Effects of desipramine maintenance on cocaine self-administration by humans, J. Pharmacol. Exp. Ther, 253 (1990) 760-770.

97 Fischman, M.W., Schuster, C.R., Javaid, J., Hatano, Y. and Davis, J., Acute tolerance development to the cardiovascular and subjective effects of cocaine, J. Pharmacol. Exp. Ther, 235 (1985) 677-682.

98 Foltin, R.W, and Fischman, M.W., Assessment of abuse liability of stimulant drugs in humans: a methodological survey, Drug Alcohol Depend., 28 (1991) 3-48.

99 Fouriezos, G. and Wise, R.A., Pimozide-induced extinction of intracranial self-stimulation: response patterns rule out motor or performance deficits, Brain Res., 103 (1976) 377-380.
100 Fujiwara, Y., Kazahaya, Y., Nakashima, M., Sato, M. and Otsuki, S., Behavioral sensitization to methamphetamine in the rat: an ontogenic study, Psychopharmacology, 91 (1987) 316-319.

101 Fung, Y.K., Effects of chronic nicotine pretreatment on (t)amphetamine and nicotine-induced synthesis and release of $\left[{ }^{3} \mathrm{H}\right]$ dopamine from $\left[{ }^{3} \mathrm{H}\right]$ tyrosine in rat nucleus accumbens, $J$. Pharm. Pharmacol., 41 (1989) 66-68.

102 Gaiardi, M., Bartoletti, M., Bacchi, A., Gubellini, C., Costa, M. and Babbini, $M_{\text {, }}$, Role of repeated exposure to morphine in determining its affective properties: Place and taste conditioning studies in rats, Psychopharmacology, 103 (1991) 183-186.

103 Gallistel, C.R., Boytim, M., Gomita, Y. and Klebanoff, L., Does pimozide block the reinforcing effect of brain stimulation?, Pharmacol. Biochem. Behav., 17 (1982) 769-781.

104 Gallistel, C.R., Shizgal, P. and Yeomans, J.S., A portrait of the substrate for self-stimulation, Psychol. Rev., 88 (1981) 228-273.

105 Gawin, F.H. and Ellinwood, E.J., Cocaine and other stimulants. Actions, abuse and treatment, N. Engl. J. Med., 318 (1988) $1173-1182$

106 Geary, N. and Smith, G.P., Pimozide decreases the positive reinforcing effect of sham fed sucrose in the rat, Pharmacol. Biochem. Behav., 22 (1985) 787-790.

107 Glick, S.D. and Hinds, P.A., Sex differences in sensitization to cocaine-induced rotation, Eur. J. Pharmacol, 99 (1984) 119-121.

108 Glick, S.D. and Hinds, P.A., Differences in amphetamine and morphine sensitivity in lateralized and non-lateralized rats: locomotor activity and drug self-administration, Eur. J. Pharmacol., $118(1985)$ 239-244.

109 Glick, S.D., Merski, C., Steindorf, S., Wang, S., Keller, R.W. and Carlson, J.N., Neurochemical predisposition to self-administer morphine in rats, Brain Res., 578 (1992) 215-220.

110 Glick, S.D., Shapiro, R.M., Drew, K.L., Hinds, P.A. and Carlson, J.N., Differences in spontaneous and amphetamine-induced rotational behavior and in sensitization to amphetamine, among Sprague-Dawley derived rats from different sources, Physiol. Behav., 38 (1986) 67-70.

111 Glickman, S.E. and Schiff, B.B., A biological theory of reinforcement, Psychol. Rev., 74 (1967) 81-109.

112 Gnegy, M.E., Hewlett, G.H.K., Yee, S.L. and Welsh, M.J., Alterations in calmodulin content and localization in areas of rat brain after repeated intermittent amphetamine, Brain Res., $562(1991) 6-12$

113 Gold, L.H., Swerdlow, N.R. and Koob, G.F., The role of mesolimbic dopamine in conditioned locomotion produced by amphetamine, Behav. Neurosci., 102 (1988) 544-552.

114 Goldstein, K.R., Knapp, D.J., Saiff, E.I., Pohorecky, L.A. and Benjamin, D., Sensitization to ethanol demonstrated in placepreference and locomotor activation, Soc Neurosci. Abstr, 18 (1992) 107.

115 Gratton, A., Wise, R.A. and Kiyatkin, E., Chronoamperometric measurements of dopamine levels in the rat nucleus accumbens during cocaine self-administration, Soc Neurosci. Abstr., 18 (1992) 1076.

116 Greenberg, B.D. and Segal, D.S., Acute and chronic behavioral interactions between phencyclidine (PCP) and amphetamine: evidence for a dopaminergic role in some PCP-induced behaviors, Pharmacol Biochem. Behav., 23 (1985) 99-105.

117 Greenberg, B.D. and Segal, D.S., Evidence for multiple opiate receptor involvement in different phencyclidine-induced unconditioned behaviors in rats, Psychopharmacology, 88 (1986) $44-53$.

118 Grill, H.J. and Norgren, R., Chronically decerebrate rats demonstrate satiation but not bait shyness, Science, 201 (1978) 267-269.

119 Grill, H.J, and Norgren, R., The taste reactivity test. I. Mimetic responses to gustatory stimuli in neurologically normal rats, Brain Res, 143 (1978) 263-279.

120 Haertzen, C.A., Kocher, T.R. and Miyasato, K., Reinforcements from the first drug experience can predict later drug habits and/or addiction: results with coffee, cigarettes, alcohol, barbiturates, minor and major tranquilizers, stimulants, marijuana, hallucinogens, heroin, opiates and cocaine, Drug Alcohol Depend., 11 (1983) 147-165. 
121 Hamamura, T., Akiyama, K., Akimoto, K., Kashihara, K., Okumura, $\mathrm{K}$., Ujike, $\mathrm{H}$. and Otsuki, S., Co-administration of either a selective D1 or D2 dopamine antagonist with methamphetamine prevents methamphetamine-induced behavioral sensitization and neurochemical change, studied by in vivo intracerebral dialysis, Brain Res., 546 (1991) 40-46.

122. Harris, G.C. and Williams, J.T., Sensitization of locus ceruleus neurons during withdrawal from chronic stimulants and antidepressants, J. Pharmacol. Exp. Ther., 261 (1992) 476-483.

123 Harsing, L.G., Sershen, H. and Laitha, A., Dopamine efflux from striatum after chronic nicotine: evidence for autoreceptor desensitization, J. Neurochem., 59 (1992) 48-54.

124 Henry, D.J. and White, F.J., Repeated cocaine administration causes persistent enhancement of D1 dopamine receptor sensitivity within the rat nucleus accumbens, J. Pharmacol. Exp. Ther., 258 (1991) 882-890.

125 Herman, J.P., Guillonneau, D., Dantzer, R., Scatton, B., Semerdjian-Rouquier, $L$. and Le Moal, M.s, Differential effects of inescapable footshocks and of stimuli previously paired with inescapable footshocks on dopamine turnover in cortical and limbic areas of the rat, Life Sci., 30 (1982) 2207-2214.

126 Hernandez, L. and Hoebel, B.G., Food reward and cocaine increase extracellular dopamine in the nucleus accumbens as measured by microdialysis, Life Sci., 42 (1988) 1705-1712.

127 Hill, R.T., Facilitation of conditioned reinforcement as a mechanism of psychomotor stimulation. In E. Costa and S. Garattini (Eds.), Amphetamine and Related Compounds, Raven Press, New York, 1970, pp. 781-795.

128 Hinson, R.E. and Poulos, C.X., Sensitization to the behavioral effects of cocaine: modification by Pavlovian conditioning, Pharmacol. Biochem. Behav., 15 (1981) 559-562.

129 Hiroi, N. and White, N.M., Conditioned stereotypy: behavioral specification of the UCS and pharmacological investigation of the neural change, Pharmacol. Biochem. Behav., 32 (1989) 249258.

130 Hiroi, N. and White, N.M., The reserpine-sensitive dopamine pool mediates $(+)$-amphetamine-conditioned reward in the place preference paradigm, Brain Res., 510 (1990) 33-42.

131 Hoebel, B.G., Neuroscience and motivation: pathways and peptides that define motivational systems. In R.C. Atkinson, R.J. Herrnstein, G. Lindzay and R.D. Lance (Eds.), Steven's Handbook of Experimental Psychology, Vol. 1, Perception and Motivation, John Wiley, New York, 1988, pp. 547-597.

132 Hooks, M.S., Colvin, A.C., Juncos, J.L. and Justice Jr., J.B., Individual differences in basal and cocaine-stimulated extracellualr dopamine in the nucleus accumbens using quantitative microdialysis, Brain Ress, 587 (1992) 306-312.

133 Hooks, M.S., Jones, G.H., Liem, B.J. and Justice Jr., J.B., Sensitization and individual differences to IP amphetamine, cocaine or caffeine following repeated intracranial amphetamine infusions, Pharmacol. Biochem. Behav, 43 (1992) 815-823.

134 Hooks, M.S., Jones, G.H., Neill, D.B. and Justice Jr., J.B., Individual differences in amphetamine sensitization: dose-dependent effects, Pharmacol. Biochem. Behav., 41 (1992) 203-210.

135 Hooks, M.S., Jones, G.H., Smith, A.D., Neill, D.B. and Justice Jr., J.B., Individual differences in locomotor activity and sensitization, Pharmacol. Biochem. Behav, 38 (1991) 467-470.

136 Hotger, B.A., Giles, M.K. and Schenk, S., Preexposure to amphetamine and nicotine predisposes rats to self-administer a low dose of cocaine, Psychopharmacology, 107 (1992) 271-276.

137 Horger, B.A., Shelton, K. and Schenk, S., Preexposure sensitizes rats to the rewarding effects of cocaine, Pharm. Biochem. Behav, 37 (1990) 707-711.

138 Horger, B.A., Wellman, P.J., Morien, A., Davies, B.T. and Schenk, S., Caffeine exposure sensitizes rats to the reinforcing effects of cocaine, Neuroreport, 2 (1991) 53-56.

139 Horvitz, J.C. and Ettenberg, A., Conditioned incentive properties of a food-paired conditioned stimulus remain intact during dopamine receptor blockade, Behav. Neurosci., 105 (1991) 536541.

$140 \mathrm{Hu}, \mathrm{X} . \mathrm{T}$. Brooderson, R.J. and White, F.J., Repeated stimulation of D1 dopamine receptors causcs time-dependent alter- ations in the sensitivity of both $\mathrm{D} 1$ and $\mathrm{D} 2$ dopamine receptors within the rat striatum, Neuroscience, 50 (1992) 137-147.

141 Hunt, H.F., Some effects of drugs on classical (type s) conditioning, Ann. NY Acad. Sci., 65 (1956) 258-267.

142 Hunt, W.A. and Lands, W.E., A role for behavioral sensitization in uncontrolled ethanol intake, Alcohol, 9 (1992) 327-328.

143 Hurd, Y.L., Weiss, F., Koob, G.F., And, N.E. and Ungerstedt, U., Cocaine reinforcement and extracellular dopamine overflow in rat nucleus accumbens: an in vivo microdialysis study, Brain Res., 498 (1989) 199-203.

144 Ichikawa, J., Changes in behavior and central monoaminergic systems in the rat after repeated methamphetamine pretreatment: presynaptic regulatory mechanism, Yakubutsu Seishin Kodo, 8 (1988) 389-403.

145 Ingram, D.K and Corfman, T.P., An overview of neurobiological comparisons in mouse strains, Neurosci. Biobehav. Rev., 4 (1980) 421-435.

146 Invernizzi, R., Pozzi, L. and Samanin, R., Release of dopamine is reduced by diazepam more in the nucleus accumbens than in the caudate nucleus of conscious rats, Neuropharmacology, 30 (1991) $575-578$

147 Iwamoto, E.T., Comparison of the pharmacologic effects of $N$-allylnormetazocine and phencyclidine: sensitization, crosssensitization and opioid antagonist activity, Psychopharmacology, 89 (1986) 221-229.

148 Jaffe, J.H., Drug addiction and drug abuse. In A.G. Gilman, T.W. Rall, A.S. Nies and P. Taylor (Eds.), The Pharmacological Basis of Therapeutics, Pergamon Press, New York, 1990, pp. $522-573$.

149 Jaffe, J.H., Current concepts of addiction. In C.P. O'Brien and J.H. Jaffe (Eds.), Addictive States, Raven Press, New York, 1992, pp. 1-21.

150 Jaffe, J.H., Cascella, N.G., Kumor, K.M. and Sherer, M.A.

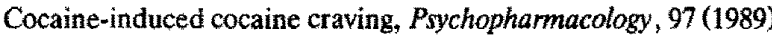
$59-64$.

151 Jones, B.E. and Prada, J.A., Drug-seeking behavior during methadone maintenance, Psychopharmacologia, 41 (1975) 7-10.

152 Jones, G.H., Marsden, C.A. and Robbins, T.W., Increased sensitivity to amphetamine and reward-related stimuli following social isolation in rats: possible disruption of dopamine-dependent mechanisms of the nucleus accumbens, Psychopharmacology, 102 (1990) 364-372.

153 Joyce, E.M. and Iversen, S.D., The effect of morphine applied locally to mesencephalic dopamine cell bodies on spontaneous motor activity in the rat, Neurosci. Lett., 14 (1979) 207-212.

154 Kalivas, P.W., Sensitization to repeated enkephalin administration into the ventral tegmental area of the rat. II. Involvement of the mesolimbic dopamine system, J. Pharmacol. Exp. Ther. 235 (1985) 544-550.

155 Kalivas, P.W. and Duffy, P., Effects of daily cocaine and morphine treatment on somatodendritic and terminal field dopamine release, $J$. Neurochem., 50 (1988) 1498-1504.

156 Kalivas, P.W. and Duffy, P., Similar effects of daily cocaine and stress on mesocorticolimbic dopamine neurotransmission in the rat, Biol. Psychiatry, 25 (1989) 913-928.

157 Kalivas, P.W. and Duffy, P., Effect of acute and daily cocaine treatment on extracellular dopamine in the nucleus accumbens, Synapse, 5 (1990) 48-58.

158 Kalivas, P.W. and Duffy, P., Time course of extracellular dopamine and behavioral sensitization to cocaine: I. Dopamine axon terminals, $J$. Neurosci., 13 (1993) 266-275.

159 Kalivas, P.W., Duffy, P., Abhold, R. and Dilts, R.P., Sensitization of mesolimbic dopamine neurons by neuropeptides and stress. In P.W. Kalivas and C.D. Barnes (Eds.), Sensitization in the Nervous System, Telford Press, Caldwell, NJ, 1988, pp. $119-143$.

160 Kalivas, P.W. and Stewart, J., Dopamine transmission in the initiation and expression of drug- and stress-induced sensitization of motor activity, Brain Res. Rev., 16 (1991) 223-244.

161 Karler, R., Calder, L.D., Chaudhry, I.A. and Turkanis, S.A. Blockade of "reverse tolerance" to cocaine and amphetamine by MK-801, Life Sci. 45 (1989) 599-606. 
162 Karler, R., Calder, L.D. and Turkanis, S.A., Changes in CNS sensitivity to cannabinoids with repeated treatment: tolerance and auxoesthesia, NIDA Res. Monogr., 54 (1984) 312-322.

163 Karler, R., Calder, L.D. and Turkanis, S.A., DNQX blockade of amphetamine behavioral sensitization, Brain Res., 552 (1991) 295-300.

164 Karler, R,, Chaudhry, I.A., Calder, L.D. and Turkanis, S.A., Amphetamine behavioral sensitization and the excitatory amino acids, Brain Res., 537 (1990) 76-82.

165 Katz, J.L. and Goldberg, S.R., Preclinical assessment of abuse liability of drugs, Agents and Actions, 23 (1988) 18-26.

166 Kazahaya, Y., Akimoto, K. and Otsuki, S., Subchronic methamphetamine treatment enhances methamphetamine- or cocaineinduced dopamine efflux in vivo,, Biol. Psychiatry, 25 (1989) 903-912.

167 Keller, R.W., Maisonneuve, I.M., Carlson, I.N. and Glick, S.D., Within-subject sensitization of striatal dopamine release after a single injection of cocaine: an in vivo microdialysis study, Synapse, 11 (1992) 28-34.

168 Kelley, A.E, and Delfs, J.M., Dopamine and conditioned reinforcement. I. Differential effects of amphetamine microinjections into striatal subregions, Psychopharmacology, 103 (1991) $187-196$.

169 Kelley, A.E. and Delfs, J.M., Dopamine and conditioned reinforcement. II. Contrasting effects of amphetamine microinjection into the nucleus accumbens with peptide microinjection into the ventral tegmental area, Psychopharmacology, 103 (1991) 197-203.

170 Khantzian, E.J., The self-medication hypothesis of addictive disorders: focus on heroin and cocaine dependence, $A m . J$. Psychiatry, 142 (1985) 1259-1264.

171 Kita, T., Okamoto, M. and Nakashima, T., Nicotine-induced sensitization to ambulatory stimulant effect produced by daily administration into the ventral tegmental area and the nucleus accumbens in rats, Life Sci., 50 (1992) 583-590.

172 Kiyatkin, E., Wise, R.A. and Gratton, A., Chronamperometic measurements of dopamine levels in the rat nucleus accumbens, Soc. Neurnsci. Abstr., 18 (1992) 374.

173 Knoblich, G., Curtis, D., Faustman, W.O., Zarcone, V., Stewart, S., Mefford, I. and King, R., Increased CSF HVA with craving in long-term abstinent cocaine abusers, Biol. Psychiatry, 32 (1992) 96-100.

174 Kokkinidis, L., Zacharko, R.M. and Anisman, H., Amphetamine withdrawal: a behavioral evaluation, Life Sci., 38 (1986) 16171623.

175 Kulta, M.G., Scalzo, F.M., Ali, S.F. and Holson, R.R., Ontogeny of the enhanced behavioral response to amphetamine in amphetamine*pretreated rats, Psychopharmacology, 100 (1990) $377-382$.

176 Kolta, M.G., Shreve, P., De Souza, V. and Uretsky, N.J., Time course of the development of the enhanced behavioral and biochemical responses to amphetamine after pretreatment with amphetamine, Neuropharmacology, 24 (1985) 823-829.

177 Kolta, M.G., Shreve, P. and Uretsky, N.J., Effect of methylphenidate pretreatment on the behavioral and biochemical responses to amphetamine, Eur. J. Pharmacol., 117 (1985) 279 282.

178 Kolta, M.G., Shreve, P. and Uretsky, N.J., Effect of pretreatment with amphetamine on the interaction between amphetamine and dopamine neurons in the nucleus accumbens, Neuropharmacology, 28 (1989) 9-14.

179 Koob, G.F., Dopamine, addiction and reward, Sem. Neurosci., 4 (1992) 139-148.

180 Kuob, G.F. and Bloom, F.E., Cellular and molecular mecha nisms of drug dependence, Science, 242 (1988) 715-723.

181 Koob, G.F. and Goeders, N.E., Neuroanatomical substrates of drug self-administration. In J.M. Liebman and S.J. Cooper (Eds.), The Neuropharmacological Basis of Reward, Oxford University Press, New York, 1989, pp. 214-263.

182 Koob, G.F., Stinus, L., Le Moal, M. and Bloom, F.E., Opponent process theory of motivation: neurobiological evidence from studies of opiate dependence, Neurosci. Biobehat: Rev., 13 (1989) $135-140$.

183 Kosobud, A.E., Harris, A.E. and Chapin, J.K., Actions of drugs of abuse on reward-related activity in neurons of the ventral tegmental area and prefrontal cortex in the rat, Soc. Neurosci. Abstr., 18 (1992) 900.

184 Kozlowski, L.T. and Wilkinson, D.A., Use and misuse of the concept of craving by alcohol, tobacco and drug researchers, $B r$. J. Addict, 82 (1987) 31-36.

185 Krieckhaus, E.E. and Wolf, $G$., Acquisition of sodium by rats: interactions of innate mechanisms and latent learning, J. Comp. Physiol. Psychol., 65 (1968) 197-201.

$186 \mathrm{Ksir}, \mathrm{C}$., Hakan, R., Hall, D.P. and Kellar, K.J., Exposure to nicotine enhances the behavioral stimulant effect of nicotine and increases binding of $\left[{ }^{3} \mathrm{H}\right]$ acetylcholine to nicotinic receptors, Neuropharmacology, 24 (1985) 527-531.

187 Kuczenski, R. and Leith, N.J., Chronic amphetamine: is dopamine a link in or mediator of the development of tolerance or reverse tolerance?, Pharmacol. Biochem. Behau, 15 (1981) $405-413$.

188 Kuczenski, R. and Segal, D.S., Psychomotor stimulant-induced sensitization: behavioral and neurochemical correlates. In P.W. Kalivas and C.D. Barnes (Eds.), Sensitization in the Nervous System, Telford Press, Caldwell, NJ, 1988, pp. 175-205.

189 Kuroki, T., Tsutsumi, T., Hirano, M., Matsumoto, T., Tatebayashi, Y., Nishiyama, K., Uchimura, H., Shiraishi, A., Nakahara, T. and Nakamura, K., Behavioral sensitization to betaphenylethylamine (PEA): enduring modifications of specific dopaminergic neuron systems in the rat, Psychopharmacology, 102 (1990) $5-10$

190 La Hoste, G.J., Mormede, P., Rivet, J.M. and Le Moal, M., Differential sensitization to amphetamine and stress responsivity as a function of inherent laterality, Brain Res., 453 (1988) 381-384.

191 Lal, H., Miksic, S., Drawbaugh, R., Numan, R. and Smith, N., Alleviation of narcotic withdrawal syndrome by conditional stimuli, Pavlov J. Biol. Sci., 11 (1976) 251-262.

192 Lamb, R.J., Preston, K.L., Schindler, C., Meisch, R.A., Davis, F., Katz, J.L., Henningfield, J.E. and Goldberg, S.R., The reinforcing and subjective effects of morphine in post-addicts: a dose-response study, J. Phamacol. Exp. Ther., 259 (1991) 11651173.

193 Lasagna, L., von Felsinger, J.M. and Beecher, H.K., Drug-induced mood changes in man. 1 . Observations on healthy subjects, chronically ill patients and "postaddicts", JAMA, 157 (1955) $1006-1020$.

194 Leith, N.J. and Kuczenski, R., Two dissociable components of behavioral sensitization following repeated amphetamine administration, Psychopharmacology, 76 (1982) 310-5.

195 Lett, B.T., Repeated exposures intensify rather than diminish the rewarding effects of amphetamine, morphine and cocaine, Psychopharmacology, 98 (1989) 357-362.

196 Leyton, M. and Stewart, J., Preexposure to foot-shock sensitizes the locomotor response to subsequent systemic morphine and intra-nucleus accumbens amphetamine, Pharmacol. Biochem. Behav., 37 (1990) 303-310.

197 Lin-Chu, G., Robinson, T.E. and Becker, J.B., Sensitization of rotational behavior produced by a single exposure to cocaine, Pharmacol. Biochent. Behav., 22 (1985) 901-903.

198 Ljungberg, T. Apicella, P. and Schultz, W., Responses of monkey midbrain dopamine neurons during delayed alternation performance, Brain Res., 567 (1991) 337-341.

199 Ljungberg, T., Apicella, P. and Schultz, W., Responses of monkey dopamine neurons during learning of hehavioral reactions, J. Neurophysiol., 67 (1992) 145-163.

200 MacLennan, A.J. and Maier, S.F., Coping and the stress-induced potentiation of stimulant stereotypy in the rat, Science, 219 (1983) $1091-1093$.

201 Magos, L., Persistence of the effect of amphetamine on stereotyped activity on rats, Eur. J. Pharmacol., 6 (1969) 200-201.

$202 \mathrm{Maj}$, J. and Wedzony, K., Repeated treatment with imipramine 
or amitriptyline increases the locomotor response of rats to (+)-amphetamine given into the nucleus accumbens, J. Pharm. Pharmacol., 37 (1985) 362-364.

$203 \mathrm{Maj}$, J., Wedzony, K. and Klimek, V., Desipramine given repeatedly enhances behavioural effects of dopamine and $d$ amphetamine injected into the nucleus accumbens, Eur. J. Pharmacol., 140 (1987) 179-185.

204 Markou, A. and Koob, G.F., Postcocaine anhedonia: an animal model of cocaine withdrawal, Neuropsychopharmacology, 4 (1991) 17-26.

205 Martin, G.M., Bechara, A. and van der Kooy, D., The perception of emotion: parallel neural processing of the affective and discriminative properties of opiates, Psychobiology, 19 (1991) 147-152.

206 Martin, S.D., Yeragani, V.K., Lodhi, R. and Galloway, M.P., Clinical ratings and plasma HVA during cocaine abstinence, Biol. Psychiatry, 26 (1989) 356-362.

207 Martin-Iverson, M.T., Ortmann, R. and Fibiger, H.C., Place preference conditioning with methylphenidate and nomifensine, Brain Res., 332 (1985) 59-67.

208 Masur, J. and Boerngen, R., The excitatory component of ethanol in mice: a chronic study, Pharmacol. Biochem. Behav., 13 (1980) 777-780.

209 Masur, J., Oliveira de Souza, M.L. and Zwicker, A.P., The excitatory effect of ethanol: absence in rats, no tolerance and increased sensitivity in mice, Pharmacol. Biochem. Behav., 24 (1986) 1225-1228.

210 Mazurski, E.J. and Beninger, R.J., Environment-specific conditioning and sensitization with (+)-amphetamine, Pharmacol. Biochem. Behav., 27 (1987) 61-65.

211 McAuliffe, W.E., A second look at first effects: the subjective effects of opiates on nonaddicts, J. Drug Issues, 5 (1975) 369-399.

212 McAuliffe, W.E., A test of Wikler's theory of relapse: the frequency of relapse due to conditioned withdrawal sickness, Int. J. Addict, 17 (1982) 19-33.

213 McAuliffe, W.E. and Gordon, R.A., A test of Lindesmith's theory of addiction: the frequency of euphoria among long-term addicts, Am. J. Sociol, 79 (1974) 795-840.

214 Meisel, R.L., Camp, D.M. and Robinson, T.E., A microdialysis study of ventral striatal dopamine during sexual behavior in female Syrian hamsters, Behav. Brain Res., in press.

215 Meyer, R.E., Conditioning phenomena and the problem of relapse in opioid addicts and alcoholics, NIDA Res. Monogr., 84 (1988) 161-179.

216 Miller, G.A., Galanter, E. and Pribram, K.H., Plans and the Structure of Behavior, Holt, Rinehart \& Winston, New York, 1965.

217 Miller, J.D., Sanghera, M.K. and German, D.C., Mesencephalic dopaminergic unit activity in the behaviorally conditioned rat, Life Sci., 29 (1981) 1255-1263.

218 Mitchell, J.B. and Stewart, J., Facilitation of sexual behaviors in the male rat associated with intra-VTA injections of opiates, Pharmacol. Biochem. Behav., 35 (1990) 643-650.

219 Mithani, S., Martin-Iverson, M.T., Phillips, A.G. and Fibiger, H.C., The effects of haloperidol on amphetamine- and methylphenidate-induced conditioned place preferences and locomotor activity, Psychopharmacology, 90 (1986) 247-252.

220 Mittleman, G., Castañeda, E., Robinson, T.E. and Valenstein, E.S., The propensity for nonregulatory ingestive behavior is related to differences in dopamine systems: behavioral and biochemical evidence, Behav. Neurosci. 100 (1986) 213-220.

221 Mittleman, G. and Valenstein, E.S., Individual differences in non-regulatory ingestive behavior and catecholamine systems, Brain Res., 348 (1985) 112-117.

222 Morency, M.A. and Beninger, R.J., Dopaminergic substrates of cocaine-induced place conditioning, Brain Res., 399 (1986) 3341.

223 Morley, J.E., Levine, A.S. and Rowland, N.E., Stress induced eating, Life Sci., 32 (1983) 2169-2182.

224 Mueller, K., Kunko, P.M., Whiteside, D. and Haskett, C., Time course of amphetamine-induced locomotor stereotypy in an open field, Psychopharmacology, 99 (1989) 501-507.
225 Nabeshima, T., Fukaya, H., Yamaguchi, K., Ishikawa, K., Furukawa, $H$. and Kameyama, $T$., Development of tolerance and supersensitivity to phencyclidine in rats after repeated administration of phencyclidine, Eur. J. Pharmacol., 135 (1987) 23-33.

226 Nakayama, M., Koyama, T. and Yamashita, I., Long-lasting decrease in dopamine uptake sites following repeated administration of methamphetamine in the rat striatum, Brain Res., 601 (1993) 209-212.

227 Nestler, E.J., Molecular mechanisms of drug addiction, $J$. Neurosci, 12 (1992) 2439-2450.

228 Nisbett, R.E. and Wilson, T.D., Telling more than we can know: verbal reports on mental processes, Psychol. Rev., 84 (1977) 231-259.

229 O'Brien, C.P., Childress, A.R., McLellan, A.T. and Ehrman, R., A learning model of addiction. In C.P. O'Brien and J.H. Jaffe (Eds.), Addictive States, Raven Press, New York, 1992, pp. 157-177.

230 Parker, L.A., Taste reactivity responses elicited by reinforcing drugs: a dose-response analysis, Behav. Neurosci., 105 (1991) 955-964.

231 Patrick, S.L., Thompson, T.L., Walker, J.M. and Patrick, R.L., Concomitant sensitization of amphetamine-induced behavioral stimulation and in vivo dopamine release from the rat caudate nucleus, Brain Res., 538 (1991) 343-346.

232 Paulson, P.E., Camp, D.M. and Robinson, T.E., The time course of transient behavioral depression and persistent behavioral sensitization in relation to regional brain monoamine concentrations during amphetamine withdrawal in rats, Psychopharmacol ogy, 103 (1991) 480-492.

233 Paulson, P.E. and Robinson, T.E., Sensitization to systemic amphetamine produces an enhanced locomotor reoponse to a subsequent intra-accumbens amphetamine in rats, Psychopharmacology, 104 (1991) 140-141.

234 Pérez-Cruet, J., Conditioning of striatal dopamine metabolism with methadone, morphine or bulbocapnine as an unconditioned stimulus, Pavlov J. Biol. Sci., 11 (1976) 237-250.

235 Pérez-de la Mora, M., López-Quiroz, D., Méndez-Franco, J. and Drucker-Colin, R., Chronic administration of amphetamine increases glutamic acid decarboxylase activity in the rat substantia nigra, Neurosci. Lett., 109 (1990) 315-320.

236 Peris, J. and Zahniser, N.R., One injection of cocaine produces a long-lasting increase in $\left[{ }^{3} \mathrm{H}\right]$-dopamine release, Pharmacol. Biochem. Behav., 27 (1987) 533-535.

237 Peris, J. and Zahniser, N.R., Persistent augmented dopamine release after acute cocaine requires dopamine receptor activation, Pharmacol. Biochem. Behav., 32 (1989) 71-76.

238 Pettit, H.O., Pan, H.T., Parsons, L.H. and Justice Jr., J.B., Extracellular concentrations of cocaine and dopamine are enhanced during chronic cocaine administration, $J$. Neurochem. 55 (1990) 798-804.

239 Phillips, A.G., Pfaus, J.G. and Blaha, C.D., Dopamine and motivated behavior: insights provided by in vivo analyses. In $P$. Willner and J. Scheel-Kruger (Eds.), The Mesolimbic Dopamine System: from Motivation to Action, John Wiley, New York, 1991, pp. 199-224.

240 Phillips, T.J. and Crabbe, J.C., Behavioral studies of genetic differences in alcohol action. In J.C. Crabbe and R.A. Harris (Eds.), The Genetic Basis of Alcohol and Drug Actions, Plenum Press, New York, 1991, pp. 25-104.

241 Piazza, P.V., Deminière, J.-M., Maccari, S., Le Moal, M., Mormède, $P$. and Simon, $H$., Individual vulnerability to drug self-administration: action of corticosterone on dopaminergic systems as a possible pathophysiological mechanism. In P. Willner and J. Scheel-Krüger (Eds.), The Mesolimbic Dopamine System: From Motivation to Action, John Wiley, New York, 1991, pp. 473-495.

242 Piazza, P.V., Deminière, J.M., Le Moal, M. and Simon, H., Factors that predict individual vulnerability to amphetamine self-administration, Science, 245 (1989) 1511-1513.

243 Piazza, P.V., Deminière, J.M., Le Moal, M. and Simon, H., Stress- and pharmacologically induced behavioral sensitization 
increases vulnerability to acquisition of amphetamine self-administration, Brain Res., 514 (1990) 22-26.

244 Piazza, P.V., Deroche, V., Rougé-Pont, F., Deminière, J.M., Maccari, S., Le Moal, M. and Simon, H., Individual differences in the sensitivity to corticostcronc's rcinforcing effects and in corticosterone-induced dopamine release may be a biological basis for sensation-seeking, Soc. Neurosci. Abstr., 18 (1992) 1076.

245 Pinel, J.P.J. and Mana, M.J., Adaptive interactions of rats with dangerous inanimate objects: support for a cognitive theory of defensive behavior. In R.J. Blanchard, P.F. Brain, D.C. Blan chard and S. Parmigiani (Eds.), Ethoexperimental Approaches to the Study of Behavior, Kluwer Academic Publishers, Dordrecht, The Netherlands, 1989, pp. 137-150.

246 Pleim, E.T., Matochik, J.A., Barfield, R.J. and Auerbach, S.B., Correlation of dopamine release in the nucleus accumbens with masculine sexual behavior in rats, Brain Res., 524 (1990) 160-163.

247 Poncelet, M., Dangoumau, L., Soubrie, P. and Simon, P., Effects of neuroleptic drugs, clonidine and lithium on the expression of conditioned behavioral excitation in rats, Psychopharmacology, 92 (1987) 393-397.

248 Post, R., Intermittent versus continuous stimulation: effect of time interval on the development of sensitization or tolerance, Life Sci., 26 (1980) 1275-1282.

249 Post, R.M., Cocaine psychoses: a continuum model, Am. J. Psychiatry, 132 (1975) 225-231.

250 Post, R.M. and Contel, N.R., Human and animal studies of cocaine: implications for development of behavioral pathology. In I. Creese (Ed.), Stimulants: Neurochemical, Behavioral and Clinical Perspectives, Raven Press, New York, 1983, pp. 169-203.

251 Post, R.M., Lockfeld, A., Squillace, K.M. and Contel, N.R., Drug-environment interaction: context dependency of cocaineinduced behavioral sensitization, Life Sci., 28 (1981) 755-760.

252 Post, R.M., Weiss, S.R., Fontana, D. and Pert, A., Conditioned sensitization to the psychomotor stimulant cocaine. In P.W. Kalivas and H.H. Samson (Eds.), Annals of the New York Academy of Sciences: The Neurobiology of Drug and Alcohol Addiction, The New York Academy of Sciences, New York, 1992, pp. 386-399.

253 Powell, J., Bradley, B. and Gray, J., Classical conditioning and cognitive determinants of subjective craving for opiates: an investigation of their relative contributions, Br. J. Addict., 87 (1992) 1133-1144

254 Randrup, A. and Munkvad, I., Stereotyped activities produced by amphetamine in several animal species and man, Psychopharmacologia, 11 (1967) 300-310.

255 Rapoport, J.L., The biology of obsessions and compulsions, Sci. Am., 260 (1989) 82-89.

256 Rapoport, J.L., Obsessive compulsive disorder and basal ganglia dysfunction, Psychol. Med., 20 (1990) 465-469.

257 Rebec, G.V., Changes in brain and behavior produced by amphetamine: a perspective based on microdialysis, voltammery and single-unit electrophysiology in freely moving animals. In R.R. Watson (Ed.), Biochemistry and Physiology of Substance Abuse, CRC Press, Boca Raton, FL, 1991, pp. 93-115.

258 Rebec, G.V. and Segal, D.S., Enhanced responsiveness to intraventricular infusion of amphetamine following its repeated systemic administration, Psychopharmacology, 62 (1979) 101-102.

259 Rescorla, R.A., Hierarchical associative relations in Pavlovian conditioning and instrumental training, Curr. Direct Psychol. Sci., 1 (1992) 66-70.

260 Risinger, F.O., Dickinson, S.D. and Cunningham, C.L. Haloperidol reduces ethanol-induced motor activity stimulation but not conditioned place greference, Psychopharmacology, 107 (1992) 453-456

261 Rivet, J.M., Stinus, L., Le Moal, M. and Mormede, P., Behavioral sensitization to amphetamine is dependent on corticosteroid receptor activation, Brain Res., 498 (1989) 149-153.

262 Robbins, T.W., Cador, M., Taylor, J.R. and Everitt, B.J., Limbic-striatal interactions in reward-related processes, Neurosci. Biobehav. Rev., 13 (1989) 155-162.

263 Robbins, T.W. and Everitt, B.J., Functions of dopamine in the dorsal and ventral striatum, Sem. Neurosci, 4 (1992) 119-127.
264 Robbins, T.W., Watson, B.A., Gaskin, M. and Ennis, C., Contrasting interactions of pipradrol, $d$-amphetamine, cocaine, cocaine analogues, apomorphine and other drugs with conditioned reinforcement, Psychopharmacology, 80 (1983) 113-119.

265 Roberts-Lewis, J.M., Roseboom, P.H., Iwaniec, L.M. and Gnegy, M.E., Differential down-regulation of D1-stimulated adenylate cyclase activity in rat forebrain after in vivo amphetamine treatments, J. Neurosci., 6 (1986) 2245-2251.

266 Robinson, J.H. and Pritchard, W.S., The meaning of addiction: reply to West, Psychopharmacology, 108 (1992) 411-416.

267 Robinson, T.E., Behavioral sensitization: characterization of enduring changes in rotational behavior produced by intermittent injections of amphetamine in male and female rats, Psychopharmacology, 84 (1984) 466-475.

268 Robinson, T.E., Stimulant drugs and stress: factors influencing individual differences in the susceptibility to sensitization. In P.W. Kalivas and C. Barnes (Eds.), Sensitization of the Nervous System, Telford Press, Caldwell, N.J., 1988, pp. 145-173.

269 Robinson, T.E., The neurobiology of amphetamine psychosis: evidence from studies with an animal model. In T. Nakazawa (Ed.), Taniguchi Symposia on Brain Sciences, Vol. 14, Biological Basis of Schizophrenia, Japan Scientific Societies Press, Tokyo, 1991, pp. 185-201.

270 Robinson, T.E., Persistent sensitizing effects of drugs on brain dopamine systems and behavior: implications for addiction and relapse. In S.G. Korenman and J.D. Barchas (Eds.), The Biological Basis of Substance Abuse, Oxford University Press, New York, in press.

271 Robinson, T.E. and Becker, J.B., Behavioral sensitization is accompanied by an enhancement in amphetamine-stimulated dopamine release from striatal tissue in vitro, Eur. J. Pharmacol., 85 (1982) 253-254.

272 Robinson, T.E. and Becker, J.B., Enduring changes in brain and behavior produced by chronic amphetamine administration: a review and evaluation of animal models of amphetamine psychosis, Brain Res. Rev., 396 (1986) 157-198.

273 Robinson, T.E., Becker, J.B. and Presty, S.K., Long-term facilitation of amphetamine-induced rotational behavior and striatal dopamine release produced by a single exposure to amphetamine: sex differences, Brain Res., 253 (1982) 231-241.

274 Robinson, T.E., Jurson, P.A., Bennett, J.A. and Bentgen, K.M., Persistent sensitization of dopamine neurotransmission in ventral striatum (nucleus accumbens) produced by past experience with (+)-amphetamine: a microdialysis study in freely moving rats, Br. Res., 462 (1988) 211-222.

275 Romo, R. and Schultz, W., Dopamine neurons of the monkey midbrain: contingencies of responses to active touch during self-initiated arm movements, J. Neurophysiol., 63 (1990) 592606 .

276 Roseboom, P.H. and Gnegy, M.E., Acute in vivo amphetamine produces a homolgous desensitization of dopamine receptorcoupled adenylate cyclase activities and decreases agonist binding to the D1 site, Mol. Pharmacol., 34 (1989) 148-156.

277 Rossetti, Z.L., D'Aquila, P.S., Hmaidan, Y., Gessa, G.L. and Serra, G., Repeated treatment with imipramine potentiates cocaine-induced dopamine release and motor stimulation, Eur. J. Pharmacol., 201 (1991) 243-245.

278 Rozin, P. and Fallon, A.E., A perspective on disgust, Psychol. Rev., 94 (1987) 23-41.

279 Sato, M., Acute exacerbation of methamphetamine psychosis and lasting dopaminergic supersensitivity - a clinical survey, Psychopharmacol. Bull., 22 (1986) 751-756.

280 Sato, M., Chen, C.C., Akiyama, K. and Otsuki, S., Acute exacerbation of paranoid psychotic state after long-term abstinence in patients with previous methamphetamine psychosis, Biol. Psychiatry, 18 (1983) 429-440.

281 Schenk, S., Lacelle, G., Gorman, K. and Amit, Z., Cocaine self-administration in rats influenced by environmental conditions: implications for the etiology of drug abuse, Neurosci. Lett., 81 (1987) 227-231.

282 Schenk, S., Valadez, A., McNamara, C. and Horger, B.A., Blockade of sensitizing effects of amphetamine preexposure on 
cocaine self-administration by the NMDA antagonist MK- 801 , Soc. Neurosci. Abstr., 18 (1992) 1237.

283 Schiff, S.R., Conditioned dopaminergic activity, Biol. Psychiatry, 17 (1982) $135-154$.

284 Schiorring, E., Amphetamine induced selective stimulation of certain behaviour items with concurrent inhibition of others in an open-field test with rats, Behaviour, 39 (1971) 1-17.

285 Schiorring, E., An open field study of stereotyped locomotor activity in amphetamine-treated rats, Psychopharmacology, 66 (1979) 281-287.

286 Schiorring, E., Psychopathology induced by "speed drugs", Pharmacol. Biochem. Behav, 1 (1981) 109-122.

287 Schultz, W., Responses of midbrain dopamine neurons to behavioral trigger stimuli in the monkey, $J$. Neurophysiol., 56 (1986) 1439-1461.

288 Schultz, W. Activity of dopamine neurons in the behaving primate, Sem. Neurosci, 4 (1992) 129-138.

289 Schultz, W. and Romo, R., Dopamine neurons of the monkey midbrain: contingencies of responses to stimuli eliciting immediate behavioral reactions, J. Neurophysiol., 63 (1990) 607-624.

290 Schuster, C., Drug-seeking behaviour: implications for theories of drug dependence. In G. Edwards and M. Lader (Eds.), The Nature of Drug Dependence, Oxford, New York, 1990, pp. 171193.

291 Segal, D.S., Behavioral and neurochemical correlates of repeated $d$-amphetamine administration, Adv. Biochem. Psychopharmacol, 13 (1975) 247-262.

292 Segal, D.S., Geyer, M.A. and Schuckit, M.A., Stimulant-induced psychosis: an evaluation of animal models, Essays Neurochem. Neuropharmacol, 5 (1981) 95-129.

293 Segal, D.S., Kelly, P.H., Koob, G. and Roberts, D.C.S., Nonstriatal dopamine mechanisms in the response to repeated $d$ amphetamine administration. In E. Usdin, 1. Kopin and $J$. Barchas (Eds.), Catecholamines: Basic and Clinical Frontiers, Vol. 2, Pergamon Press, New York, 1979, pp. 1672-1674.

294 Segal, D.S. and Kuczenski, R., Individual differences in responsiveness to single and repeated amphetamine administration: behavioral characteristics and neurochemical correlates, J. Pharmacol. Exp. Ther., 242 (1987) 917-926.

295 Segal, D.S. and Kuczenski, R., In vivo microdialysis reveals a diminished amphetamine-induced DA response corresponding to behavioral sensitization produced by repeated amphetamine pretreatment, Brain Res., 571 (1992) 330-337.

296 Segal, D.S. and Kuczenski, R, Repeated cocaine administration induces behavioral sensitization and corresponding decreased extracellular dopamine responses in caudate and accumbens, Brain Res., 577 (1992) 351-355.

297 Segal, D.S. and Schuckit, M.A., Animal models of stimulant-induced psychosis. In I. Creese (Ed), Stimulants: Neurochemical, Behavioral and Clinical Perspectives, Raven Press, New York, 1983, pp. 131-167.

298 Sherer, M.A., Intravenous cocaine: psychiatric effects, biological mechanisms, Biol. Psychiatry, 24 (1988) 865-885.

299 Shippenberg, T.S., Emmett-Oglesby, M.W., Ayesta, F.J. and Herz, A., Tolerance and selective cross-tolerance to the motivational effects of opioids, Psychopharmacology, 96 (1988) 110-115.

300 Short, P.H. and Shuster, L., Changes in brain norepinephrine associated with sensitization to $d$-amphetamine, Psychophamacology, 48 (1976) 59-67.

301 Shuster, L., Hudson, J., Anton, M. and Righi, D., Sensitization of mice to methylphenidate, Psychopharmacology, 77 (1982) $31-36$.

302 Shuster, L., Webster, G.W. and $Y u, G$, Increased running response to morphine in morphine-pretreated mice, $J$. Pharmacol. Exp. Ther., 192 (1975) 64-67.

303 Shuster, L., Webster, G.W. and $Y u$, G., Perinatal narcotic addiction in mice: sensitization to morphine stimulation, Addict. Dis., 2 (1975) 277-292.

304 Shuster, L., Yu, G. and Bates, A., Sensitization to cocaine stimulation in mice, Psychopharmacology, 52 (1977) 185-190.

305 Siegel, S., Learning and psychopharmacology. In M.E. Jarvik
(Ed.), Psychopharmacology in the Practice of Medicine, Appleton-Century-Crofts, New York, 1977.

306 Siegel, S., Drug anticipation and drug tolerance. In M. Lader (Ed.), The Psychopharmacology of Addiction, Oxford University Press, New York, 1988, pp. 73-97.

307 Skinner, B.F., Are theories of learning necessary?, Psychol. Rev., 57 (1950) 193-216.

308 Smith, G.P. and Schneider, L.H., Relationships between mesolimbic dopamine function and eating behavior, $A n n$. NY Acad. Sci, 537 (1988) 254-261.

309 Smith, J.E., Co, C., Freeman, M.E. and Lane, J.D., Brain neurotransmitter turnover correlated with morphine-seeking behavior of rats, Pharmacol. Biochem. Behav., 16 (1982) 509-519.

310 Smith, J.E. and Dworkin, S.I., Behavioral contingencies determine changes in drug-induced transmitter turnover, Drug Dev. Res., 20 (1990) 337-348.

311 Söderpalm, B., Svensson, L., Hulthe, P., Johannessen, K. and Engel, J.A., Evidence for a role for dopamine in the diazepam locomotor stimulating effect, Psychopharmacology, 104 (1991) 97-102.

312 Solomon, R.L., Acquired motivation and affective opponentprocesses. In J. Madden (Ed.), Neurobiology of Learning, Emotion and Affect, Raven Press, New York, 1991, pp. 307-348.

313 Sorensen, S.M., Hattox, S., Johnson, S.W., Bickford, P., Murphy, R. and Freedman, R., Norepinephrine-dependent and independent mechanisms of persistent effects of amphetamine in rat cerebellum, Life Sci., 36 (1985) 2383-2389.

314 Sorensen, S.M., Johnson, S.W. and Freedman, R., Persistent effects of amphetamine on cerebellar Purkinje neurons following chronic administration, Brain Res., 247 (1982) 365-371.

315 Sorg, B.A. and Kalivas, P.W., Effects of cocaine and footshock stress on extracellular dopamine levels in the ventral striatum, Brain Res., 559 (1991) 29-36.

316 Spanos, L.J. and Yamamoto, B.K., Acute and subchronic effects of methylenedioxy-methamphetamine [(+/-)MDMA] on locomotion and serotonin syndrome behavior in the rat, Pharmacol. Biochem. Behav., 32 (1989) 835-840 [published erratum appears in Pharmacol. Biochem. Behav. 1989, 34:679].

317 Spyraki, C., Fibiger, H.C. and Phillips, A.G., Cocaine-induced place preference conditioning: lack of effects of neuroleptics and 6-hydroxydopamine lesions, Brain Res, 253 (1982) 195-203.

318 Squire, L.R. and Zola-Morgan, S., The medial temporal lobe memory system, Science, 253 (1991) 1380-1386.

319 Steiner, J.E., Human facial expressions in response to taste and smell stimulation, Adv. Child Dev. Behav., 13 (1979) 257-295.

320 Steketee, J.D., Sorg, B.A. and Kalivas, P.W., The role of the nucleus accumbens in sensitization to drugs of abuse, Prog. Neuropsychopharmacol. Biol. Psychiatry, 16 (1992) 237-246.

321 Stevens, J.R., An anatomy of schizophrenia?, Arch. Gen. Psychiatry, 29 (1973) 177-189.

322 Stewart, J., Reinstatement of heroin and cocaine self-administration behavior in the rat by intracerebral application of morphine in the ventral tegmental area, Pharmacol. Biochem. Behav., 20 (1984) 917-923.

323 Stewart. J., Conditioned stimulus control of the expression of sensitization of the behavioral activating effects of opiate and stimulant drugs. In I. Gormezano and E.A. Wasserman (Eds.), Learning and Memory: The Behavioral and Biological Substrates, Erlbaum, Hillsdale, NJ, 1992, pp. 129-151.

324 Stewart, J., Tolerance and sensitization to the behavioral effects of drugs. In P. Nencini (Ed.), Controllo Farmacologico del Comportamento, Unione Tipografico, Torino, Italy, 1992, pp. 161183.

325 Stewart, J. and De Wit, H., Reinstatement of drug-taking behavior as a method of assessing incentive motivational properties of drugs. In M.A. Bozarth (Ed.), Methods of Assessing the Reinforcing Properties of Abused Drugs, Springer-Verlag, New York, 1987, pp. 211-227.

326 Stewart, J., de Wit, H. and Eikelboom, R., Role of unconditioned and conditioned drug effects in the self-administration of opiates and stimulants, Psychol. Rev., 91 (1984) 251-268. 
327 Stewart, J. and Vezina, P., A comparison of the effects of intra-accumbens injections of amphetamine and morphine on reinstatement of heroin intravenous self-administration behavior, Brain Res., 457 (1988) 287-294.

328 Stewart, J. and Vezina, P., Extinction procedures abolish conditioned stimulus control but spare sensitized responding to amphetamine, Behat: Pharmacol., 2 (1991) 65-71.

329 Stewart, J. and Wise, R.A., Reinstatement of heroin self-administration habits: morphine prompts and naltrexone discourages renewed responding after extinction, Psychopharmacology, 108 (1992) 79-84.

330 Strecker, R.E., Steinfels, G.F. and Jacobs, B.L., Dopaminergic unit activity in freely moving cats: lack of relationship to feeding, satiety and glucose injections, Brain Res., 260 (1983) 317-321.

331 Swerdlow, N.R., Braff, D.I., Masten, V.L. and Geyer, M.A., Schizophrenic-like sensorimotor gating abnormalities in rats following dopamine infusion into the nucleus accumbens, Psychopharmacology, 101 (1990) 414-420.

332 Swerdlow, N.R. and Koob, G.F., Dopamine, schizophrenia, mania and depression: toward a unified hypothesis of corticostriato-pallido-thalamic function, Behav. Brain Sci., 10 (1987) 197-245.

333 Taylor, J.R. and Robbins, T.W., Enhanced behavioural control by conditioned reinforcers following microinjections of $d$ amphetamine into the nucleus accumbens, Psychopharmacology, 84 (1984) 405-412.

334 Taylor, J.R. and Robbins, T.W., 6-Hydroxydopamine lesions of the nucleus accumbens, but not of the caudate nucleus, attenuate enhanced responding with reward-related stimuli produced by intra-accumbens $d$-amphetamine, Psychopharmacology, 90 (1986) 390-397.

335 Ternes, J.W., Ehrman, R.N. and O'Brien, C.P., Nondependent monkeys self-administer hydromorphone, Behal. Neurosci., 99 (1985) $583-588$.

336 Thierry, A.M., Tassin, J.P., Blanc, G. and Glowinski, J., Selective activation of the mesocortical DA system by stress, Nature, 263 (1976) 242-243.

337 Tiffany, S.T., A cognitive model of drug urges and drug-use behavior: role of automatic and nonautomatic processes, $P_{s y}$ chol. Rev., 97 (1990) 147-168.

338 Tilson, H.A. and Rech, R.A., Conditioned drug effects and absence of tolerance to $d$-amphetamine induced motor activity, Pharmacol. Biochem. Behav., 1 (1973) 149-153.

339 Toates, F., Motivational Systems, Cambridge University Press, Cambridge, 1986.

340 Treit, D. and Berridge, K.C., A comparison of benzodiazepine, serotonin and dopamine agents in the taste-reactivity paradigm, Pharmacol. Biochem. Behau., 37 (1990) 451-456.

341 Trulson, M.E. and Preussler, D.W., Dopamine-containing ventral tegmental area neurons in freely moving cats: activity during the slccp-waking cycle and effects of stress, Exp. Neurol., 83 (1984) 367-377.

342 Ujike, H., Tsuchida, H., Kanzaki, A., Akiyama, K. and Otsuki, S., Competitive and non-competitive $N$-methyl-D-aspartate antagonists fail to prevent the induction of methamphetamine-induced sensitization, Life Sci., 50 (1992) 1673-1681.

343 Vaccarino, F.J., Schiff, B.B. and Glickman, S.E., Biological view of reinforcement. In S.B. Klein and R.R. Mowrer (Eds.), Contempory Learning Theories, Erlbaum, Hillsdale, NJ, 1989, pp. 111-142.

344 Vezina, P. and Stewart, J., Conditioning and place-specific sensitization of increases in activity induced by morphine in the VTA, Pharmacol. Biochem. Behau., 20 (1984) 925-934.

345 Vezina, P. and Stewart, J., The effect of dopamine receptor blockade on the development of sensitization to the locomotor activating effects of amphetamine and morphine, Brain Res., 499 (1989) 108-120.

346 Vezina, P. and Stewart, J., Amphetamine administered to the ventral tegmental area but not to the nucleus accumbens sensitizes rats to systemic morphine: lack of conditioned effects, Brain Res., 516 (1990) 99-106.
347 Vezina, P. and Zurakowski, B., Prior exposure to intra-VTA amphetamine sensitizes the nucleus accumbens dopaminergic response to systemic amphetamine as measured by invivo microdialysis, Soc. Neurosci. Abstr., 18 (1992) 720.

348 Walter, S. and Kuschinsky, K., Conditioning of morphine-induced locomotor activity and stereotyped behaviour in rats, $I$. Neural Transm. Gen. Sect., 78 (1989) 231-247.

349 Watson, S.J., Trujillo, K.A., Herman, J.P. and Akil, H., Neuroanatomical and neurochemical substrates of drug-seeking behavior: overview and future directions. In A. Goldstein (Ed.), Molecular and Cellular Aspects of the Drug Addictions, SpringerVerlag, New York, 1989, pp. 29-91.

350 Weiskrantz, L., Warrington, E.K., Sanders, M.D. and Marshall, $\mathrm{J}$., Visual capacity in the hemianopic field following a restricted occipital ablation, Brain, 97 (1974) 709-728.

351 Weiss, F., Markou, A., Lorang, M.T. and Koob, G.F., Basal extracellular dopamine levels in the nucleus accumbens are decreased during cocaine withdrawal after unlimited-access self-administration, Brain Res., 593 (1992) 314-318.

352 Weiss, S.R., Post, R.M., Pert, A., Woodward, R. and Murman, D., Context-dependent cocaine sensitization: differential effect of haloperidol on development versus expression, Pharmacol. Biochem. Behal., 34 (1989) 655-661.

353 Whishaw, I.Q. and Kornelsen, R.A., Two types of action revealed by ibotenic acid nucleus accumbens lesions: dissociation of food carrying and hoarding and the role of primary incentive motivation, Behal. Brain Res., in press.

354 White, F.J. and Wolf, M.E., Psychomotor stimulants. In J. Pratt (Ed.), The Biological Bases of Drug Tolerance and Dependence, Academic Press, New York, 1991, pp. 153-197.

355 White, N., Sklar, L. and Amit, Z., The reinforcing action of morphine and its paradoxical side effect, Psychopharmacology, 52 (1977) 63-66.

356 White, N.M. and Milner, P.M., The psychobiology of reinforcers, Annu. Rev. Psychol, 43 (1992) 443-471.

357 Wikler, A., Recent progress in research on the neurophysiological basis of morphine addiction, Am. J. Psychiatry, 105 (1948) $329-338$.

358 Wilcox, R.A., Robinson, T.E. and Becker, J.B., Enduring enhancement in amphetamine-stimulated striatal dopamine release in vitro produced by prior expusure to amphetamine or stress in vivo, Eur. J. Pharmacol, 124 (1986) 375-376.

359 Wise, R.A., Catecholamine theories of reward: a critical review, Brain Res., 152 (1978) 215-247.

360 Wise, R.A., Neuroleptics and operant behavior: the anhedonia hypothesis, Behav. Brain Sci., 5 (1982) 39-87.

361 Wise, R.A., The anhedonia hypothesis: Mark III, Behav. Brain Sci., 8 (1985) 178-186.

362 Wise, R.A., The role of reward pathways in the development of drug dependence, Pharmacol. Ther., 35 (1987) 227-263.

363 Wise, R.A., The neurobiology of craving: implications for the understanding and treatment of addiction, J. Abnorm. Psychol, 97 (1988) 118-132.

364 Wise, K.A. and Bozarth, M.A., Brain mechanisms of drug reward and euphoria, Psychiatry Med., 3 (1985) 445-460.

365 Wise, R.A. and Bozarth, M.A., A psychomotor stimulant theory of addiction, Psychol. Rev., 94 (1987) 469-492.

366 Wise, R.A. and Hoffman, D.C., Localization of drug reward mechanisms by intracranial injections, Synapse, 10 (1992) 247263.

367 Wise, R.A., Murray, A. and Bozarth, M.A., Bromocriptine selfadministration and bromocriptine-reinstatement of cocainetrained and heroin-trained lever pressing in rats, Psychopharmacology, 100 (1990) 355-360.

368 Wise, R.A. and Rompre, P.-P., Brain dopamine and reward, Annu. Rev. Psychol., 40 (1989) 191-225.

369 Wolf, M.E. and Khansa, M.R., Repeated administration of MK-801 produces sensitization to its own locomotor stimulant effects but blocks sensitization to amphetamine, Brain Res., 562 (1991) 164-168.

370 Wolf, M.E., White, F.J., Nassar, R., Brooderson, R.J. and 
Khansa, M.R., Differential development of autoreceptor subsensitivity and enhanced dopamine release during amphetamine sensitization, J. Pharmacol. Exp. Ther., 264 (1993) 249-1255.

371 Woods, J.H., Katz, J.L. and Winger, G., Use and abuse of benzodiazepines, J. Am. Med. Assoc., 260 (1988) 3476-3480.

372 Woods, J.H. and Schuster, C.R., Reinforcement properties of morphine, cocaine and SPA as a function of unit dose, Int. $J$. Addict., 3 (1968) 231-237.

373 Woolverton, W.L., Cervo, L. and Johanson, C.E., Effects of repeated methamphetamine administration on methamphetamine self-administration in rhesus monkeys, Pharmacol. Biochem. Behav., 21 (1984) 737-741.

374 Xenakis, S. and Sclafani, A., The dopaminergic mediation of a sweet reward in normal and VMH hyperphagic rats, Pharmacol. Biochem. Behav. 16 (1982) 293-302.
375 Yamada, S., Kojima, H., Yokoo, H., Tsutsumi, T., Takamuki, K., Anraku, S., Nishi, S. and Inanaga, K., Enhancement of dopamine release from striatal slices of rats that were subchronically treated with methamphetamine, Biol. Psychiatry, 24 (1988) 399408.

376 Young, A.M., Joseph, M.H. and Gray, J.A., Increased dopamine release in vivo in nucleus accumbens and caudate nucleus of the rat during drinking: a microdialysis study, Neuroscience, 48 (1992) 871-876.

377 Young, A.M.J., Gray, J.A. and Joseph, M.H., Dopamine function in the nucleus accumbens in conditioning studied by microdialysis, Abstracts of the 7th International Catecholamine Symposium in Amsterdam, (1992) 360. 